\title{
Rational curves on hypersurfaces of low degree, II
}

\author{
Joe Harris and Jason Starr
}

\begin{abstract}
This is the second in a sequence of papers on the geometry of spaces of rational curves of degree $e$ on a general hypersurface $X \subset \mathbb{P}^{n}$ of degree $d$. In Part I (J. reine angew. Math. 571 (2004), 73-106) it is proved that, if $d<(n+1) / 2$, then for each $e$ the space of rational curves is irreducible, reduced and has the expected dimension. In this paper it is proved that, if $d^{2}+d+1 \leqslant n$, then for each $e$ the space of rational curves is a rationally connected variety; in particular it has negative Kodaira dimension.
\end{abstract}

\section{Contents}

1 Statement of results

2 Deformation ampleness $\quad 39$

3 Deformation theory of stable maps 45

4 Conditions on families of stable maps

5 The induction argument $\quad 66$

6 Twistable lines on hypersurfaces $\quad 76$

7 Base case of the induction for hypersurfaces $\quad 80$

8 Proof of the main theorem $\quad 91$

References $\quad 92$

\section{Statement of results}

Consider a general hypersurface $X \subset \mathbb{P}^{n}$. Let $d=\operatorname{deg}(X)$. In [HRS04] it is proved that, if $d<$ $(n+1) / 2$, then for each $e$ the space of rational curves of degree $e$ is irreducible, reduced and has the expected dimension. The main result of this paper is the following theorem.

TheOREM 1.1. If $X \subset \mathbb{P}^{n}$ is a general hypersurface of degree $d$ and if $n \geqslant d^{2}+d+1$ then for each integer $e \geqslant 1$ the stack $\overline{\mathcal{M}}_{0,0}(X, e)$ is rationally connected. More precisely, there exists a morphism $f: \mathbb{P}^{1} \rightarrow\left(\left(\bar{M}_{0,0}(X, e)\right)_{\text {fine }}\right)_{\mathrm{sm}}$ such that $f^{*} T_{\bar{M}_{0,0}(X, e)}$ is ample.

Remark 1.2.

i) The scheme $\bar{M}_{0,0}(X, e)$ is the coarse moduli space of $\overline{\mathcal{M}}_{0,0}(X, e)$, the open subset $\left(\bar{M}_{0,0}\right.$ $(X, e))_{\text {fine }}$ is the fine moduli locus, and the open subset $\left(\left(\bar{M}_{0,0}(X, e)\right)_{\text {fine }}\right)_{\mathrm{sm}}$ is the smooth locus of the fine moduli locus.

ii) For the cases $d=1,2$, a related and stronger theorem is proved in [KP01, Theorem 3]; namely the coarse moduli space $\bar{M}_{0,0}(X, e)$ is rational. The proof relies on the fact that $X$ is a homogeneous space and does not extend to the case $d \geqslant 3$. Moreover, it is not clear from [KP01] that there exists a very free rational curve in the smooth locus of the fine moduli locus of the coarse moduli space.

Received 2 December 2002, accepted in final form 16 January 2004, published online 1 December 2004. 2000 Mathematics Subject Classification 14C05 (primary), 14E08 (secondary).

Keywords: Kontsevich moduli space, stable map, rationally connected variety.

This journal is (C) Foundation Compositio Mathematica 2005. 


\section{J. HARRIS AND J. STARR}

The space $\overline{\mathcal{M}}_{0,0}(X, e)$ is the Kontsevich moduli stack of stable maps, which will be recalled below; it is a Deligne-Mumford stack containing the parameter space of smooth rational curves in $X$ of degree $e$ as an open substack. A variety is rationally connected if any two closed points are contained in the image of a morphism from $\mathbb{P}^{1}$ to the variety. Rationally connected varieties have negative Kodaira dimension; hence the schemes $\bar{M}_{0,0}(X, e)$ have negative Kodaira dimension.

The motivation behind Theorem 1.1 is a conjectural relationship between rational connectedness of $\overline{\mathcal{M}}_{0,0}(X, e)$ and a theorem of Lang about rational points of varieties defined over the function field of a surface.

Theorem 1.3 (Lang [Lan52]). Let $K$ be the function field of a surface over $\mathbb{C}$ and let $X \subset \mathbb{P}_{K}^{n}$ be a hypersurface of degree $d$. If $d^{2} \leqslant n$, then $X(K) \neq \emptyset$.

There is a naive parameter count that suggests that if $d^{2} \leqslant n$ then for $e \gg 0$ the stack $\overline{\mathcal{M}}_{0,0}(X, e)$ is rationally connected, and if $d^{2} \geqslant n+2$ then for $e \gg 0$ the stack $\overline{\mathcal{M}}_{0,0}(X, e)$ is of general type; i.e. the Kodaira dimension of $\overline{\mathcal{M}}_{0,0}(X, e)$ is determined by the same inequality as in Lang's theorem. This suggests that Lang's theorem is related to rational connectedness of spaces of rational curves. In a personal communication, A. J. de Jong has outlined an approach for proving that a $K$-variety $X$ has a $K$-point if the stacks $\overline{\mathcal{M}}_{0,0}\left(X \otimes_{K} \bar{K}, e\right)$ are rationally connected, and a certain Brauer obstruction vanishes. Of course Lang's original proof is simple and direct. But de Jong's approach could apply to classes of varieties where Lang's proof does not apply, i.e. to varieties that are not hypersurfaces in projective space.

The naive parameter count is not rigorous. As it seems impossible to make it rigorous, it is not recalled here. In this paper a different strategy is developed for proving that the stacks $\overline{\mathcal{M}}_{0,0}(X, e)$ are rationally connected, and this strategy is applied to hypersurfaces in projective space. This strategy should also apply to other varieties; hence it is formulated in greater generality than strictly needed for the case of hypersurfaces.

\subsection{The Kontsevich moduli space}

The most natural parameter space for rational curves of degree $e$ on $X$ is the open subscheme of the Hilbert scheme parametrizing smooth rational curves of degree $e$ on $X, R_{X}^{e} \subset$ Hilb $_{X}^{e t+1}$. For $e>1, R_{X}^{e}$ is not proper. To study the global geometry of $R_{X}^{e}$, e.g. to determine its Kodaira dimension, it is necessary to embed it as an open subset of a proper scheme. The simplest choice is to take the closure $\overline{R_{X}^{e}}$ inside Hilb ${ }_{X}^{e t+1}$. This is a poor choice for two reasons: First, there is no simple characterization of the closed subschemes of $X$ that correspond to points in $\overline{R_{X}^{e}}$. Second, the deformation theory of a closed subscheme of $X$ is difficult to work with.

There is a better choice, one where the points of the closure have a simple geometric meaning, and where the deformation theory is easier to work with. This choice is $\overline{\mathcal{M}}_{0,0}(X, e)$, or more generally $\overline{\mathcal{M}}_{0, r}(X, e)$, the Kontsevich moduli space of degree e, r-pointed, genus-0 stable maps to $X$. This space has one disadvantage over $\overline{R_{X}^{e}}$; namely $\overline{\mathcal{M}}_{0, r}(X, e)$ is a Deligne-Mumford stack rather than a scheme. However, the coarse moduli space $\bar{M}_{0, r}(X, e)$ is a projective scheme; cf. [Ale96]. For the reader unfamiliar with stacks, most occurrences of $\overline{\mathcal{M}}_{0, r}(X, e)$ can safely be replaced by $\left(\bar{M}_{0, r}(X, e)\right)_{\text {fine }}$, the fine moduli locus of $\bar{M}_{0, r}(X, e)$.

To be precise, $\overline{\mathcal{M}}_{0, r}(X, e)$ is the stack whose objects are triples

$$
\zeta=\left(\left(p: \Sigma \rightarrow B, \sigma_{1}, \ldots, \sigma_{r}\right), g: \Sigma \rightarrow X\right),
$$

consisting of a flat, proper family of curves $p: \Sigma \rightarrow B$, a collection of $r$ disjoint sections $\sigma_{i}: B \rightarrow \Sigma$, $i=1, \ldots, r$, with image in the smooth locus of $p$, and a morphism $g: \Sigma \rightarrow X$ such that for each geometric point $b \in B$, the fiber $\Sigma_{b}$ is a connected, at-worst-nodal curve of arithmetic genus 0 , the morphism $g_{b}: \Sigma_{b} \rightarrow X \otimes_{\mathbb{C}} \kappa(b)$ has no infinitesimal automorphism fixing the marked points $\sigma_{i}(b)$, and the degree of $\left(g_{b}\right)^{*} \mathcal{O}_{X}(1)$ is $e$ (cf. [FP95, BM96]). 


\section{RATIONAL CURVES ON HYPERSURFACES, II}

A refinement of this stack used in this paper is the Behrend-Manin stack, $\overline{\mathcal{M}}(X, \tau)$, associated to a genus-0 stable A-graph $\tau$; cf. [BM96]. A genus-0 stable $A$-graph is a tree with tails with a degree associated to each vertex that satisfies a certain stability condition. A tree is a graph that contains no cycles. A tail, or a half-edge, is an edge that originates on a vertex but does not terminate on a vertex (e.g. what one would get if one were to 'cut in half' an edge of a usual graph). A degree function is an assignment of a nonnegative integer to each vertex of the graph. The stability condition is that no vertex has both degree 0 and valence less than 3 (tails count toward the valence of a vertex).

Given an at-worst-nodal curve of genus $0, \Sigma$, a collection of marked points on $\Sigma, \sigma_{1}, \ldots, \sigma_{r}$, and a morphism $g: \Sigma \rightarrow X$, there is an associated $A$-graph defined as follows. The tree is the dual graph of $\Sigma$; there is one vertex for each irreducible component of $\Sigma$ and one edge for each node of $\Sigma$. For each marked point $\sigma_{i}$ of $\Sigma$ there is a tail attached to the obvious vertex. The degree of a vertex is simply the degree of $g^{*} \mathcal{O}(1)$ on the corresponding irreducible component. The Behrend-Manin stack $\overline{\mathcal{M}}(X, \tau)$ (essentially) parametrizes the closure of the locally closed substack of $\overline{\mathcal{M}}_{0, r}(X, e)$ of stable maps whose associated $A$-graph equals $\tau$. For the precise definition, see [BM96].

The boundary of the stack $\overline{\mathcal{M}}(X, \tau)$ is a union of stacks $\overline{\mathcal{M}}(X, \sigma)$ where $\sigma$ ranges over stable $A$-graphs such that there is a contraction from $\sigma$ to $\tau$, i.e. the graph $\sigma$ is 'more degenerate' than the graph $\tau$; cf. [BM96]. Hence the boundary of every Behrend-Manin stack can be understood inductively starting from the 'most degenerate' graphs $\tau$, for which $\overline{\mathcal{M}}(X, \tau)$ is a fiber product over $X$ of the space of pointed lines on $X$. So questions about $\overline{\mathcal{M}}(X, \tau)$ that can be studied by specializing to points in the boundary eventually reduce to questions about the space of pointed lines on $X$. Moreover, the deformation theory of a point in $\overline{\mathcal{M}}(X, \tau)$ is straightforward; it will be recalled in $\S 3$.

\subsection{Sketch of the proof}

The proof of Theorem 1.1 uses a theorem of Kollár.

Theorem 1.4 (Kollár, Theorem IV.3.7 [Kol96]). Let $V$ be an irreducible, projective variety, and let $V_{\mathrm{sm}} \subset V$ denote the smooth locus of $V$. If there exists a very free morphism $f: \mathbb{P}^{1} \rightarrow V_{\mathrm{sm}}$, i.e. a morphism such that $f^{*} T_{V}$ is an ample vector bundle, then $V$ is rationally connected.

The reader is warned that 'very free' is the first of a multitude of definitions with 'very' similar names: free, very free, deformation ample, very stable, unobstructed, twisting, very twisting, twistable, very twistable, positive, very positive, inducting pair, inductable, modification, typical, and $c$-generating linear system. A morphism $f: \mathbb{P}^{1} \rightarrow V$ is free if $f^{*} T_{V}$ is a vector bundle that is generated by global sections. The goal is to prove that there exists a very free morphism to $\overline{\mathcal{M}}_{0,0}(X, e)$ for all $e$. It is difficult to construct a very free morphism directly. However, existence of a very free morphism can be studied by specializing to the boundary of $\overline{\mathcal{M}}_{0,0}(X, e)$, and, using an induction argument, can ultimately be reduced to a question about pointed lines on $X$. The induction argument does not produce a very free morphism to the boundary of $\overline{\mathcal{M}}_{0,0}(X, e)$, but rather a reducible rational curve $B$ in the boundary such that the restriction of $T_{\overline{\mathcal{M}}_{0,0}(X, e)}$ to $B$ is deformation ample: for a deformation of $B$ to an irreducible rational curve $B^{\prime}$, the restriction of $T_{\overline{\mathcal{M}}_{0,0}(X, e)}$ to $B^{\prime}$ is ample; cf. $\S 2$ (a curve $B^{\prime}$ is called a deformation of $B$ if both $B$ and $B^{\prime}$ have a common generization). The rational curve $B^{\prime}$ is a very free rational curve which proves that $\overline{\mathcal{M}}_{0,0}(X, e)$ is rationally connected.

The induction argument constructs a reducible rational curve $B$ which itself parametrizes stable maps from reducible rational curves to $X$. Each of these stable maps is the union of a map of degree $e-1, g: \Sigma \rightarrow X$, and a line $L \subset X$ that intersect in a node $\sigma \in \Sigma \cap L$. Since the union is stable, the 1-pointed map $(\Sigma, \sigma, g)$ is stable. To simplify the deformation theory, it is assumed that $(\Sigma, \sigma, g)$ is very stable: the unmarked map $(\Sigma, g)$ is stable (this assumption must be justified!). 


\section{J. HARRIS AND J. STARR}

To guarantee that $B$ is in the smooth locus of $\overline{\mathcal{M}}_{0,0}(X, e)$, it is assumed that $(\Sigma, g)$ is unobstructed: $\mathbb{E x t}_{\mathcal{O}_{\Sigma}}^{1}\left(L_{g}, \mathcal{O}_{\Sigma}\right)=\{0\}$.

The condition that $\left.T_{\overline{\mathcal{M}}_{0,0}(X, e)}\right|_{B}$ is deformation ample can be translated into a condition on the family of pointed lines, $(L, \sigma)$, together with a condition on the family of pointed maps of degree $e-1$, $(\Sigma, \sigma, g)$; these conditions are defined in $\S 4$. The condition on the family of pointed lines is that it is very twisting. The family is twisting (respectively very twisting) if the associated morphism $\zeta: B \rightarrow \overline{\mathcal{M}}_{0,1}(X, 1)$ pulls back the vertical tangent bundle of the projection pr $: \overline{\mathcal{M}}_{0,1}(X, 1) \rightarrow$ $\overline{\mathcal{M}}_{0,0}(X, 1)$ to a bundle which is generated by global sections, and pulls back the vertical tangent bundle of the 'evaluation at the marked point' morphism ev $: \overline{\mathcal{M}}_{0,1}(X, 1) \rightarrow X$ to a bundle which is generated by global sections (respectively deformation ample). Composing $\zeta$ with ev gives a map $h: B \rightarrow X$. Such a map which arises from a twisting (respectively very twisting) family is called twistable (respectively very twistable). The property of being twistable (respectively very twistable) is an open condition on the family of all maps.

The condition on the family of pointed maps of degree $e-1$ is that it is very positive. The family is positive (respectively very positive) if the associated morphism $\zeta: B \rightarrow \overline{\mathcal{M}}_{0,1}(X, e-1)$ pulls back the vertical tangent bundle of the projection pr: $\overline{\mathcal{M}}_{0,1}(X, 1) \rightarrow \overline{\mathcal{M}}_{0,0}(X, 1)$ to a bundle which is generated by global sections (respectively ample) and the pullback by $\operatorname{pr} \circ \zeta$ of $T_{\overline{\mathcal{M}}_{0,0}(X, 1)}$ is deformation ample. The main observation is this: For a positive (respectively very positive) family over a smooth rational curve, the morphism $\zeta: B \rightarrow \overline{\mathcal{M}}_{0,1}(X, e-1)$ is free (respectively very free).

An inducting pair consists of a very twisting family of pointed lines over $B$ and a very positive family of pointed maps of degree $e-1$ over $B$ which intersect along the marked points. The induction step proves that, if an inducting pair exists for degree $e$, then an inducting pair exists for degree $e+1$. It is sometimes useful to 'forget' the very twisting family of pointed lines, and only 'remember' that the family of marked points is a very twistable map. A very positive family of pointed maps of degree $e-1$ such that the family of marked points is a very twistable map is an inductable family.

The induction step begins with an inducting pair for degree $e$ and produces an inductable family for degree $e+1$. In particular, the inductable family is very positive and there is a deformation to a very free morphism from an irreducible curve to $\overline{\mathcal{M}}_{0,1}(X, e)$ whose projection to $\overline{\mathcal{M}}_{0,0}(X, e)$ is a very free morphism. The family of unmarked maps over $B$ is obtained by gluing the very twisting family of lines and the very positive family of maps of degree $e-1$ along the curve of marked points $\sigma$. To get a family of marked maps, let $\sigma^{\prime}$ be an irreducible curve in the total space $\Sigma$ of the very positive family which is linearly equivalent to $\sigma$. The curve $\sigma^{\prime}$ fails to be a family of marked points precisely at the finitely many points in $B$ over which the curve $\sigma^{\prime}$ intersects the curve $\sigma$. The solution is to blow up each of these finitely many points on $\Sigma$; this produces a family of marked stable maps of degree $e$. Unfortunately blowing-up destroys the 'very positivity' of the family. Very positivity is restored by making a modification at each of the finitely many points of $B$ over which $\Sigma$ is blown up. The modification attaches a $\mathbb{P}^{1}$ to $B$ at the specified point and extends the family over this $\mathbb{P}^{1}$ so that the stabilized family of unmarked maps is a constant family, but such that the marked points in this constant family vary. After modification, the family of marked stable maps is inductable. Incidentally, it is the process of modification that requires using reducible curves in $\overline{\mathcal{M}}_{0,0}(X, e)$ and working with the property of deformation ample bundles.

The last section establishes the base case of the induction argument: existence of a family of pointed lines that is both very twisting and very positive. The total space of a very positive family of lines is a scroll $\Sigma$. The whole argument reduces to producing a pair of a scroll $\Sigma$ and a hypersurface $X$ containing $\Sigma$ such that $\Sigma$ corresponds to a very positive family of lines on $X$. This reduces to a computation of the dimension of certain linear systems on the scroll $\Sigma$, c-generating linear systems. This is a straightforward computation in the Cox homogeneous coordinate ring of the scroll. The hypothesis that $d^{2}+d+1 \leqslant n$ is used in this last computation. 


\section{RATIONAL CURVES ON HYPERSURFACES, II}

\subsection{Technical hypotheses}

In the proof of the induction step, some technical hypotheses are used.

Hypothesis 1.5. For each contraction of genus-0 stable $A$-graphs, $\phi: \sigma \rightarrow \tau$, the image of the morphism of Behrend-Manin stacks $\overline{\mathcal{M}}(X, \sigma) \rightarrow \overline{\mathcal{M}}(X, \tau)$ has codimension $\operatorname{dim}(X, \tau)-\operatorname{dim}(X, \sigma)$ in $\overline{\mathcal{M}}(X, \tau)$.

By [HRS04, Proposition 7.4], if $d<(n+1) / 2$ and if $X \subset \mathbb{P}^{n}$ is a general hypersurface of degree $d$, each stack $\overline{\mathcal{M}}(X, \sigma)$ has the expected dimension; thus Hypothesis 1.5 holds for $X$.

Hypothesis 1.6. A general fiber of the evaluation map ev $: \overline{\mathcal{M}}_{0,1}(X, 1) \rightarrow X$ is irreducible.

For a pair $(X, p)$ consisting of a hypersurface $X \subset \mathbb{P}^{n}$ of degree $d$ and a point $p \in X$, the associated fiber of ev is a subvariety $Z \subset \mathbb{P}^{n-1}$ which is a complete intersection of a sequence of hypersurfaces $Y_{1}, \ldots, Y_{d}$ in $\mathbb{P}^{n}$ with $\operatorname{deg}\left(Y_{i}\right)=i$ : the defining equation of $Y_{i}$ is the degree- $i$ homogeneous part of the Taylor expansion of the defining equation of $X$ about the point $p$. If the pair $(X, p)$ is general, the sequence of hypersurfaces $Y_{1}, \ldots, Y_{d}$ is general. By the Bertini theorem [Jou83, Theorems 4.10, 6.10] the intersection $Y_{1} \cap \cdots \cap Y_{d}$ is smooth and connected if $d \leqslant n-2$.

Hypothesis 1.7. For each integer $e \geqslant 0$, the locus in $\overline{\mathcal{M}}_{0,1}(X, e)$ parametrizing stable maps with nontrivial automorphism group has codimension at least 2 .

Of course any stable map with nontrivial automorphism group has an irreducible component which is a multiple cover of its image. In light of [HRS04, Proposition 7.4], a simple parameter count shows that if $d \leqslant(n+1) / 2$ and if $X \subset \mathbb{P}^{n}$ is a general hypersurface of degree $d$, then Hypothesis 1.7 is satisfied.

\subsection{Conventions}

Unless stated otherwise, schemes are of finite type and separated over Spec $\mathbb{C}$. Absolute fiber products of schemes will be fiber products over Spec $\mathbb{C}$. Absolute fiber products of stacks will be 2-fibered products over Spec $\mathbb{C}$.

\section{Deformation ampleness}

Let $T$ be a scheme.

Definition 2.1. A family of prestable curves of genus $g$ over $T$ is a proper, flat morphism $\pi: B \rightarrow T$ such that every geometric fiber of $\pi$ is a connected, at-worst-nodal curve of arithmetic genus $g$.

Notation 2.2. Let $\pi: B \rightarrow T$ be a morphism of schemes and let $E$ be a quasi-coherent sheaf on $B$. Denote by $\mu_{E}: \pi^{*} \pi_{*} E \rightarrow E$ the morphism left adjoint to the identity morphism $\pi_{*} E \rightarrow \pi_{*} E$. The sheaf $E$ is $\pi$-relatively generated by global sections if $\mu_{E}$ is surjective.

Let $\pi: B \rightarrow T$ be a family of prestable curves of genus 0 and let $E$ be a coherent sheaf on $B$. Let $\sigma: T \rightarrow B$ be a section of $\pi$ and let $\mathcal{I} \subset \mathcal{O}_{B}$ be the ideal sheaf of $\sigma(T)$.

LEMMA 2.3.

i) If $E$ is $\pi$-relatively generated by global sections, then $R^{1} \pi_{*} E=\{0\}$.

ii) If $E$ is $\pi$-relatively generated by global sections, then $R^{1} \pi_{*}(\mathcal{I} \cdot E)=\{0\}$.

iii) Let $E^{\prime}$ and $E^{\prime \prime}$ be coherent sheaves on $B$ and let

$$
0 \longrightarrow E^{\prime} \longrightarrow E \longrightarrow E^{\prime \prime} \longrightarrow 0
$$

be a short exact sequence of coherent sheaves. If $E^{\prime}$ and $E^{\prime \prime}$ are $\pi$-relatively generated by global sections, then $E$ is $\pi$-relatively generated by global sections. 


\section{J. HARRIS AND J. STARR}

Proof. All three statements can be proved locally over $T$. Hence it suffices to consider the case that $T$ is affine.

i) Since $E$ is $\pi$-relatively generated by global sections and $T$ is affine, $E$ is generated by global sections, i.e. there is a short exact sequence of coherent sheaves,

$$
0 \longrightarrow K \longrightarrow \mathcal{O}_{B}^{\oplus N} \longrightarrow E \longrightarrow 0 .
$$

Since $\pi$ is of relative dimension $1, R^{2} \pi_{*} K=\{0\}$. Since the fibers of $B$ are connected of arithmetic genus $0, R^{1} \pi_{*} \mathcal{O}_{B}=\{0\}$. In the long exact sequence of higher direct images associated to the short exact sequence above, $R^{1} \pi_{*} E$ fits between $R^{1} \pi_{*} \mathcal{O}_{B}^{\oplus N}$ and $R^{2} \pi_{*} K$; hence $R^{1} \pi_{*} E=\{0\}$.

ii) There is a short exact sequence of coherent sheaves,

$$
0 \longrightarrow \mathcal{I} \cdot E \longrightarrow E \longrightarrow E \otimes_{\mathcal{O}_{B}} \mathcal{O}_{\sigma(T)} \longrightarrow 0,
$$

giving rise to a long exact sequence of cohomology groups,

$$
\pi_{*} E \longrightarrow \pi_{*}\left(E \otimes_{\mathcal{O}_{B}} \mathcal{O}_{\sigma(T)}\right) \longrightarrow R^{1} \pi_{*}\left(\mathcal{I}_{b} \cdot E\right) \longrightarrow R^{1} \pi_{*} E .
$$

By the last paragraph, $R^{1} \pi_{*} E=\{0\}$. Since $E$ is generated by global sections,

$$
\pi_{*} E \longrightarrow \pi_{*}\left(E \otimes_{\mathcal{O}_{B}} \mathcal{O}_{\sigma(T)}\right)
$$

is surjective. Therefore $R^{1} \pi_{*}(\mathcal{I} \cdot E)=\{0\}$.

iii) By part i, $h^{1}\left(B, E^{\prime}\right)=0$; therefore every global section of $E^{\prime \prime}$ is the image of a global section of $E$. So the global sections of $E$ generate $E^{\prime \prime}$, and the global sections of $E^{\prime}$ generate $E^{\prime}$. Therefore $E$ is generated by global sections.

Let $B$ be a prestable curve of genus 0 , and let $E$ be a locally free sheaf of positive rank on $B$. A smoothing of the pair $(B, E)$ over a discrete valuation $\operatorname{ring} R$ is a pair $(\mathcal{B}, \mathcal{E})$ consisting of a family $\mathcal{B} \rightarrow$ Spec $R$ of prestable curves of genus 0 and a locally free sheaf $\mathcal{E}$ such that the generic fiber of $\mathcal{B}$ is a smooth curve, such that the closed fiber of $\mathcal{B}$ is isomorphic to $B$, and such that the restriction of $\mathcal{E}$ to the closed fiber is isomorphic to $E$. What conditions on $(B, E)$ guarantee that, for every smoothing $(\mathcal{B}, \mathcal{E})$, the restriction of $\mathcal{E}$ to the generic fiber is an ample locally free sheaf? Certainly if $E$ is ample, this is true. But $E$ need not be ample for this condition to hold: e.g. if $E$ is an invertible sheaf such that the total degree of $E$ is positive, then for every smoothing the restriction of $\mathcal{E}$ to the generic fiber is ample. Although it is not the most general criterion, the following criterion is used in the rest of this paper.

Definition 2.4. Let $B$ be a connected, proper, at-worst-nodal curve of arithmetic genus 0. A locally free sheaf $E$ on $B$ with positive rank is deformation ample if

i) $E$ is generated by global sections, and

ii) $h^{1}\left(B, E\left(K_{B}\right)\right)=0$, where $\mathcal{O}_{B}\left(K_{B}\right)$ is the dualizing sheaf of $B$.

Remark 2.5.

i) Conditions $\mathrm{i}$ and ii in Definition 2.4 are independent.

ii) If $E$ is invertible, then $E$ is deformation ample if and only if the restriction of $E$ to every irreducible component has nonnegative degree and the restriction to at least one irreducible component has positive degree; cf. Lemma 2.11.

iii) One can determine whether $E$ is deformation ample in terms of the splitting type of the restriction of $E$ to each irreducible component together with the patching isomorphisms at the nodes of $B$. 


\section{RATIONAL CURVES ON HYPERSURFACES, II}

Let $T$ be a scheme, let $\pi: B \rightarrow T$ be a family of prestable curves of genus 0 , and let $E$ be a locally free sheaf of positive rank on $B$.

DEFINITION 2.6. The sheaf $E$ is $\pi$-relatively deformation ample (or simply deformation ample if $\pi$ is understood) if

i) $E$ is $\pi$-relatively generated by global sections, and

ii) $R^{1} \pi_{*}\left(E\left(K_{\pi}\right)\right)=\{0\}$, where $\mathcal{O}_{B}\left(K_{\pi}\right)$ is the relative dualizing sheaf of $\pi$.

Let $\pi: B \rightarrow T$ be a proper, flat family of connected, at-worst-nodal curves of arithmetic genus 0 , let $E$ be a locally free sheaf on $B$ of positive rank, and let $f: T^{\prime} \rightarrow T$ be a morphism of schemes. Denote the fiber product as in the following diagram.

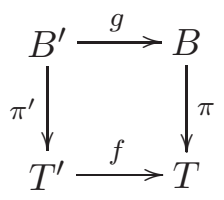

Denote by $E^{\prime}$ the pullback $g^{*} E$.

Lemma 2.7. If $E$ is $\pi$-relatively deformation ample, then $E^{\prime}$ is $\pi^{\prime}$-relatively deformation ample. If $f$ is surjective, the converse also holds.

Proof. $(\Rightarrow)$ For the main direction, by [GD64, § 8.5.2, Proposition 8.9.1], it suffices to consider the case when $T$ and $T^{\prime}$ are Noetherian affine schemes.

There is a canonical map of $\mathcal{O}_{T^{\prime}}$-modules, $\nu: f^{*} \pi_{*} E \rightarrow\left(\pi^{\prime}\right)_{*} g^{*} E$, which fits into the following commutative diagram.

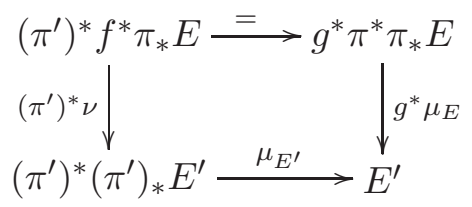

Since $\mu_{E}$ is surjective, $g^{*} \mu_{E}$ is surjective. Hence also $\mu_{E^{\prime}}$ is surjective, i.e. $E^{\prime}$ is $\pi^{\prime}$-relatively generated by global sections.

Since $\pi$ has relative dimension $1, R^{2} \pi_{*} E\left(K_{\pi}\right)=\{0\}$. By [Har77, Theorem III.12.11(b)], for every closed point $t \in T, h^{1}\left(B_{t},\left.E\left(K_{\pi}\right)\right|_{B_{t}}\right)=0$. By [Har77, Proposition III.9.3], for every closed point $t^{\prime} \in T^{\prime}, h^{1}\left(B_{t^{\prime}}^{\prime},\left.E^{\prime}\left(K_{\pi^{\prime}}\right)\right|_{B_{t^{\prime}}^{\prime}}\right)=0$. So by [Har77, Theorem III.12.11(a)] and Nakayama's lemma, $R^{1} \pi_{*}^{\prime}\left(E^{\prime}\left(K_{\pi^{\prime}}\right)\right)=\{0\}$. Hence $E^{\prime}$ is $\pi^{\prime}$-relatively deformation ample.

$(\Leftarrow)$ Now suppose that $f$ is surjective and that $E^{\prime}$ is $\pi^{\prime}$-relatively deformation ample. As above, it suffices to consider the case when $T$ and $T^{\prime}$ are Noetherian affine schemes. As above, for every closed point $t^{\prime} \in T^{\prime}, h^{1}\left(B_{t^{\prime}}^{\prime},\left.E^{\prime}\left(K_{\pi^{\prime}}\right)\right|_{B_{t^{\prime}}^{\prime}}\right)=0$. Since $T^{\prime} \rightarrow T$ is surjective, by [Har77, Proposition III.9.3] for every closed point $t \in T, h^{1}\left(B_{t},\left.E\left(K_{\pi}\right)\right|_{B_{t}}\right)=0$. So by [Har77, Theorem III.12.11(a)] and Nakayama's lemma, $R^{1} \pi_{*}\left(E\left(K_{\pi}\right)\right)=\{0\}$.

It remains to prove that $E$ is $\pi$-relatively generated by global sections. For every closed point $t \in T$, there is a closed point $t^{\prime} \in T^{\prime}$ mapping to $t$. Since $\left.E^{\prime}\right|_{B_{t^{\prime}}^{\prime}}$ is generated by global sections, also $\left.E\right|_{B_{t}}$ is generated by global sections. By Lemma $2.3, h^{1}\left(B_{t},\left.E\right|_{B_{t}}\right)=0$. By $[\operatorname{Har} 77$, Theorem III.12.11(a)] and Nakayama's lemma, $R^{1} \pi_{*}(E)=\{0\}$.

The claim is that for any coherent $\mathcal{O}_{T}$-module $\mathcal{F}, R^{1} \pi_{*}\left(\pi^{*} \mathcal{F} \otimes E\right)=\{0\}$. This is local on $T$. Locally on $T, \mathcal{F}$ is the cokernel of $\mathcal{O}_{T}^{\oplus N}$ for some $N$, so $\pi^{*} \mathcal{F} \otimes E$ is the cokernel of $E^{\oplus N}$. Since $\pi$ has relative dimension $1, R^{1} \pi_{*}$ is right exact on the category of coherent $\mathcal{O}_{B}$-modules. Since $R^{1} \pi_{*}\left(E^{\oplus N}\right)=\{0\}$, also $R^{1} \pi_{*}\left(\pi^{*} \mathcal{F} \otimes E\right)=\{0\}$, which proves the claim. 


\section{J. HARRIS AND J. STARR}

In particular, applying the long exact sequence of higher direct images to the short exact sequence,

$$
\left.0 \longrightarrow \pi^{*} \mathcal{I}_{t} \otimes E \longrightarrow E \longrightarrow E\right|_{B_{t}} \longrightarrow 0
$$

$\pi_{*}(E) \rightarrow H^{0}\left(B_{t},\left.E\right|_{B_{t}}\right)$ is surjective. Since $\left.E\right|_{B_{t}}$ is generated by global sections for every closed point $t \in T, E$ is $\pi$-relatively generated by global sections. So $E$ is $\pi$-relatively deformation ample.

Lemma 2.8. Let $\pi: B \rightarrow T$ be a proper, flat family of connected, smooth curves of genus 0 and let $E$ be a locally free sheaf of positive rank. Then $E$ is $\pi$-relatively deformation ample if and only if $E$ is $\pi$-relatively ample.

Proof. Both properties are local on $T$ and can be checked after étale, surjective base change of $T$. So it suffices to consider the case when $\pi: B \rightarrow T$ is isomorphic to $\pi_{T}: T \times \mathbb{P}^{1} \rightarrow T$.

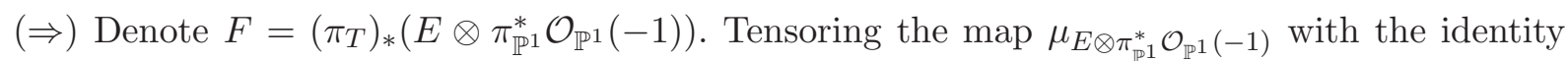
map on $\pi^{*} \mathcal{O}_{\mathbb{P}^{1}}(-1)$ gives a map $\nu: \pi_{T}^{*} F \otimes \pi_{\mathbb{P}^{1}}^{*} \mathcal{O}_{\mathbb{P}^{1}}(1) \rightarrow E$. Assume that $E$ is deformation ample. The claim is that $\nu$ is surjective. To prove this, it suffices to prove the following:

i) for every geometric point $t$ of $T, h^{1}\left(\mathbb{P}_{\kappa(t)}^{1},\left.E\right|_{B_{t}} \otimes \mathcal{O}_{\mathbb{P}^{1}}(-1)\right)=0$,

ii) $F \otimes \mathcal{O}_{T} \kappa(t)=H^{0}\left(\mathbb{P}_{\kappa(t)}^{1},\left.E\right|_{B_{t}} \otimes \mathcal{O}_{\mathbb{P}^{1}}(-1)\right)$, and

iii) the map $\left.H^{0}\left(\mathbb{P}_{\kappa(t)}^{1},\left.E\right|_{B_{t}} \otimes \mathcal{O}_{\mathbb{P}^{1}}(-1)\right) \otimes \mathcal{O}_{\mathbb{P}^{1}}(1) \rightarrow E\right|_{B_{t}}$ is surjective.

By Grothendieck's lemma [Har77, Exercise V.2.6], $\left.E\right|_{B_{t}}$ splits as a direct sum $\mathcal{O}_{\mathbb{P}^{1}}\left(a_{1}\right) \oplus \cdots \oplus$ $\mathcal{O}_{\mathbb{P}^{1}}\left(a_{r}\right)$ for some integers $a_{1} \leqslant \cdots \leqslant a_{r}$. By Lemma $2.7,\left.E\right|_{B_{t}}$ is deformation ample, and in particular $h^{1}\left(\mathbb{P}^{1},\left.E\right|_{B_{t}}(-2)\right)=0$. Hence $a_{1} \geqslant 1$, and $h^{1}\left(\mathbb{P}^{1},\left.E\right|_{B_{t}} \otimes \mathcal{O}_{\mathbb{P}^{1}}(-1)\right)=0$, i.e. part i holds. By [Har77, Theorem III.12.11(b)], also part ii holds. Finally, for $a_{i} \geqslant 1, H^{0}\left(\mathbb{P}_{\kappa}^{1}, \mathcal{O}_{\mathbb{P}^{1}}\left(a_{i}-1\right)\right) \otimes \mathcal{O}_{\mathbb{P}^{1}}(1) \rightarrow \mathcal{O}_{\mathbb{P}^{1}}\left(a_{i}\right)$ is surjective. Thus part iii holds and the claim is proved.

Now $\pi_{T}^{*} F \otimes \pi_{\mathbb{P}^{1}}^{*} \mathcal{O}_{\mathbb{P}^{1}}(1)$ is $\pi_{T^{-}}$-relatively ample. Since $E$ is a quotient of $\pi_{T}^{*} F \otimes \pi_{\mathbb{P}^{1}}^{*} \mathcal{O}_{\mathbb{P}^{1}}(1)$, also $E$ is $\pi_{T}$-relatively ample; cf. Lemma 2.10 , part i.

$(\Leftarrow)$ The converse direction follows in the same way.

Lemma 2.9. There exists an open subscheme $i: U \rightarrow T$ with the following property: for every morphism $f: T^{\prime} \rightarrow T, f\left(T^{\prime}\right)$ is contained in $U$ if and only if $E^{\prime}$ is $\pi^{\prime}$-relatively deformation ample.

Proof. By [GD64, $\S 8.5 .2$, Proposition 8.9.1], it suffices to consider the case that $T$ and $T^{\prime}$ are Noetherian affine schemes.

Let $Z_{1} \subset T$ be the closed subset,

$$
Z_{1}=f\left[\operatorname{Supp}\left(\operatorname{coker}\left(\pi^{*} \pi_{*} E \rightarrow E\right)\right)\right] .
$$

Let $Z_{2} \subset T$ be the closed subset,

$$
Z_{2}=\operatorname{Supp}\left(R^{1} \pi_{*}\left(E\left(K_{\pi}\right)\right)\right)
$$

Let $Z_{3} \subset T$ be the closed subset,

$$
Z_{3}=\operatorname{Supp}\left(R^{1} \pi_{*} E\right)
$$

Let $i: U \rightarrow T$ be the open complement of $Z_{1} \cup Z_{2} \cup Z_{3}$.

Let $f: T^{\prime} \rightarrow T$ be a morphism of Noetherian affine schemes. By [Har77, Theorem III.12.11, Proposition III.9.3] and Nakayama's lemma, $R^{1} \pi_{*}^{\prime}\left(E^{\prime}\left(K_{\pi^{\prime}}\right)\right)=\{0\}$ if and only if for each closed point $t^{\prime} \in T^{\prime}, h^{1}\left(B_{t^{\prime}}^{\prime},\left.E^{\prime}\left(K_{\pi^{\prime}}\right)\right|_{B_{t^{\prime}}^{\prime \prime}}\right)=0$. Denoting $t=f\left(t^{\prime}\right), h^{1}\left(B_{t^{\prime}}^{\prime},\left.E^{\prime}\left(K_{\pi^{\prime}}\right)\right|_{B_{t^{\prime}}^{\prime}}\right)=0$ if and only if $h^{1}\left(B_{t},\left.E\left(K_{\pi}\right)\right|_{B_{t}}\right)=0$, i.e. if and only if $t$ is contained in the complement of $Z_{2}$. Hence $R^{1} \pi_{*}^{\prime}\left(E^{\prime}\left(K_{\pi^{\prime}}\right)\right)=\{0\}$ if and only if $f(T)$ is contained in the complement of $Z_{2}$. 


\section{RATIONAL CURVES ON HYPERSURFACES, II}

By the same argument as in the converse direction of the proof of Lemma $2.7, E^{\prime}$ is $\pi^{\prime}$-relatively generated by global sections if and only if for every closed point $t^{\prime} \in T^{\prime},\left.E^{\prime}\right|_{B_{t^{\prime}}^{\prime}}$ is generated by global sections. Denoting $t=f\left(t^{\prime}\right),\left.E^{\prime}\right|_{B_{t^{\prime}}^{\prime}}$ is generated by global sections if and only if $\left.E\right|_{B_{t}}$ is generated by global sections. If $\left.E\right|_{B_{t}}$ is generated by global sections, then $h^{1}\left(B_{t},\left.E\right|_{B_{t}}\right)=0$. By [Har77, Theorem III.12.11], $t$ is not in $Z_{3}$ and $t$ is not in $Z_{1}$. Conversely, if $t$ is not in $Z_{3}$, then $\left.E\right|_{B_{t}}$ is generated by global sections if and only if $t$ is not in $Z_{1}$. Thus $E^{\prime}$ is $\pi^{\prime}$-relatively generated by global sections if and only if $f\left(T^{\prime}\right)$ is contained in the complement of $Z_{1} \cup Z_{3}$. So $E^{\prime}$ is $\pi^{\prime}$-relatively deformation ample if and only if $f\left(T^{\prime}\right)$ is contained in $U$.

LEMMA 2.10.

i) If $\chi: E \rightarrow E^{\prime \prime}$ is a morphism of locally free sheaves on $B$ whose cokernel is torsion in every fiber (in particular, if $\chi$ is surjective), if $E^{\prime \prime}$ is nonzero, and if $E$ is $\pi$-relatively deformation ample, then also $E^{\prime \prime}$ is $\pi$-relatively deformation ample.

ii) If $E^{\prime}$ and $E^{\prime \prime}$ are $\pi$-relatively deformation ample, then for every short exact sequence of coherent $\mathcal{O}_{B}$-modules,

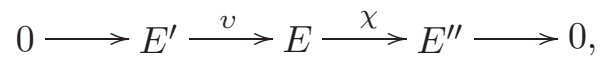

$E$ is $\pi$-relatively deformation ample.

iii) If $E$ is $\pi$-relatively deformation ample, then for every integer $n \geqslant 1$, also $E^{\otimes n}$ is $\pi$-relatively deformation ample.

Proof. i) Let $Q$ denote the cokernel of $\chi$ and let $I \subset E^{\prime \prime}$ denote the image of $\chi$. There is a short exact sequence of coherent $\mathcal{O}_{B}$-modules:

$$
0 \longrightarrow I \longrightarrow E^{\prime \prime} \longrightarrow Q \longrightarrow 0 .
$$

When we 'twist' this exact sequence by $\mathcal{O}_{B}\left(K_{\pi}\right)$, it remains exact. Because $\pi$ has relative dimension $1, R^{1} \pi_{*}$ is right exact on the category of coherent $\mathcal{O}_{B}$-modules. In particular, since $R^{1} \pi_{*} E\left(K_{\pi}\right)=\{0\}$, also $R^{1} \pi_{*} I\left(K_{\pi}\right)=\{0\}$. Since $Q$ is torsion in every fiber, $R^{1} \pi_{*} Q\left(K_{\pi}\right)=\{0\}$. Thus, by the long exact sequence of higher direct images associated to the twisted exact sequence above, $R^{1} \pi_{*} E^{\prime \prime}\left(K_{\pi}\right)=\{0\}$.

The surjective composition map

$$
\pi^{*} \pi_{*} E_{1} \stackrel{\mu_{E}}{\longrightarrow} E_{1} \longrightarrow I
$$

factors through the natural map $\mu_{I}: \pi^{*} \pi_{*} I \rightarrow I$. Hence $\mu_{I}$ is surjective, i.e. $I$ is $\pi$-relatively generated by global sections. Since $Q$ is torsion in every fiber, the support of $Q$ is finite over $T$ and it follows that $Q$ is $\pi$-relatively generated by global sections. By Lemma 2.3, part iii, $E^{\prime \prime}$ is $\pi$-relatively generated by global sections. So $E^{\prime \prime}$ is $\pi$-relatively deformation ample.

ii) By hypothesis, $R^{1} \pi_{*} E^{\prime}\left(K_{\pi}\right)=R^{1} \pi_{*} E^{\prime \prime}\left(K_{\pi}\right)=\{0\}$. By the long exact sequence of higher direct images, also $R^{1} \pi_{*} E\left(K_{\pi}\right)=\{0\}$. By Lemma 2.3, part iii, $E$ is $\pi$-relatively generated by global sections. So $E$ is $\pi$-relatively deformation ample.

iii) This is proved by induction on $n$, the case $n=1$ being tautological. It suffices to consider the case when $T$ is affine. Suppose $n>1$ and suppose the result is known for $n-1$. In particular, $E^{\otimes(n-1)}$ is generated by global sections. There is a natural surjection

$$
\pi^{*} \pi_{*}\left(E^{\otimes(n-1)}\right) \otimes \mathcal{O}_{B} E \longrightarrow E^{\otimes n}
$$

There is a surjective map $\mathcal{O}_{T}^{\oplus N} \rightarrow \pi_{*}\left(E^{\otimes(n-1)}\right)$. Hence there is a surjection $E^{\oplus N} \rightarrow E^{\otimes n}$. By part ii and induction, $E^{\oplus N}$ is $\pi$-relatively deformation ample. By part i, the quotient $E^{\otimes n}$ is $\pi$-relatively deformation ample. Thus part iii is proved by induction. 


\section{J. HARRIS AND J. STARR}

Lemma 2.11. Let $B$ be a proper, connected, at-worst-nodal curve of arithmetic genus 0 over an algebraically closed field $k$. Let $E$ be a locally free sheaf of positive rank such that:

i) for every irreducible component $B_{i} \subset B,\left.E\right|_{B_{i}}$ is generated by global sections, and

ii) there exists a nonempty, connected, closed subcurve $B^{\prime} \subset B$ such that $\left.E\right|_{B^{\prime}}$ is deformation ample.

Then $E$ is deformation ample.

Proof. Let $\delta$ be the number of irreducible components of $B$ which are not contained in $B^{\prime}$. The result is proved by induction on $\delta$. The base case $\delta=0$ is tautological, for then $B=B^{\prime}$. Assume that $\delta>0$ and that the result is true for all smaller values of $\delta$.

Let $B_{1} \subset B$ be an irreducible component of $B$. Let $B_{2} \subset B$ denote the union of all irreducible components other than $B_{1}$. There exists an irreducible component $B_{1}$ not contained in $B^{\prime}$ such that $B_{2}$ is connected: if the dual graph of $B^{\prime}$ contains every leaf (= vertex of valence 1 ) of the dual graph of $B$, then the two graphs are equal. The intersection $B_{1} \cap B_{2}$ is a single node, denoted $b$. By the induction hypothesis, $\left.E\right|_{B_{2}}$ is deformation ample.

The claim is that $E$ is generated by global sections. Denote by $F \subset E$ the image of

$$
H^{0}(B, E) \otimes_{k} \mathcal{O}_{B} \longrightarrow E .
$$

There is a short exact sequence of coherent sheaves:

$$
0 \longrightarrow E \otimes \mathcal{O}_{B} \mathcal{O}_{B_{1}}(-b) \longrightarrow E \longrightarrow E \otimes_{\mathcal{O}_{B}} \mathcal{O}_{B_{2}} \longrightarrow 0 .
$$

Since $\left.E\right|_{B_{1}}$ is a locally free sheaf on $\mathbb{P}^{1}$ generated by global sections, Grothendieck's lemma and the cohomology of line bundles on $\mathbb{P}^{1}$ imply that $h^{1}\left(B, E \otimes_{\mathcal{O}_{B}} \mathcal{O}_{B_{1}}(-b)\right)=0\left(h^{1}\left(\mathbb{P}^{1}, \mathcal{O}_{\mathbb{P}^{1}}(a)\right)=0\right.$ for $a \geqslant-1)$. Hence all the global sections of $\left.E\right|_{B_{2}}$ lift to global sections of $E$, i.e. $\left.F \rightarrow E\right|_{B_{2}}$ is surjective. So $E / F$ is supported on $B_{1}$. Hence $E / F$ is a quotient of $\left.E\right|_{B_{1}}$. Since $\left.E\right|_{B_{1}}$ is generated by global sections, also $E / F$ is generated by global sections. There is a short exact sequence

$$
0 \longrightarrow F \longrightarrow E \longrightarrow E / F \longrightarrow 0 \text {. }
$$

By Lemma 2.3, part iii, $E$ is generated by global sections.

There is a short exact sequence of coherent sheaves,

$$
0 \longrightarrow E\left(K_{B}\right) \otimes_{\mathcal{O}_{B}} \mathcal{O}_{B_{2}}(-b) \longrightarrow E\left(K_{B}\right) \longrightarrow E\left(K_{B}\right) \otimes_{\mathcal{O}_{B}} \mathcal{O}_{B_{1}} \longrightarrow 0 .
$$

This gives a long exact sequence in cohomology, part of which is,

$$
H^{1}\left(B, E\left(K_{B}\right) \otimes_{\mathcal{O}_{B}} \mathcal{O}_{B_{2}}(-b)\right) \longrightarrow H^{1}\left(B, E\left(K_{B}\right)\right) \longrightarrow H^{1}\left(B, E\left(K_{B}\right) \otimes_{\mathcal{O}_{B}} \mathcal{O}_{B_{1}}\right) \longrightarrow 0 .
$$

The inclusion map $B_{2} \rightarrow B$ is finite, and therefore there is a canonical isomorphism of $\mathcal{O}_{B}$-modules (cf. [Har66, § III.6] and [KM98, Corollary 5.68]),

$$
\mathcal{O}_{B_{2}}\left(K_{B_{2}}\right) \cong \operatorname{Hom}_{\mathcal{O}_{B}}\left(\mathcal{O}_{B_{2}}, \mathcal{O}_{B}\left(K_{B}\right)\right) \cong \mathcal{O}_{B}\left(K_{B}\right) \otimes_{\mathcal{O}_{B}} \mathcal{O}_{B_{2}}(-b) .
$$

Hence $h^{1}\left(B, E\left(K_{B}\right) \otimes_{\mathcal{O}_{B}} \mathcal{O}_{B_{2}}(-b)\right)=h^{1}\left(B_{2}, E \otimes_{\mathcal{O}_{B}} \mathcal{O}_{B_{2}}\left(K_{B_{2}}\right)\right)$, which is zero by the induction assumption. Similarly, $E\left(K_{B}\right) \otimes_{\mathcal{O}_{B}} \mathcal{O}_{B_{1}}$ is isomorphic to $E \otimes_{\mathcal{O}_{B}} \mathcal{O}_{B_{1}}(-1)$ (identifying $B_{1}$ with $\mathbb{P}^{1}$ ). Since $\left.E\right|_{B_{1}}$ is generated by global sections, it follows by Grothendieck's lemma and the cohomology of line bundles on $\mathbb{P}^{1}$ that $h^{1}\left(B_{1},\left.E\right|_{B_{1}}(-1)\right)=0$. Hence $h^{1}\left(B, E\left(K_{B}\right)\right)=0$, and $E$ is deformation ample. Therefore the lemma is proved by induction on $\delta$.

Remark 2.12. A particular case of Lemma 2.11 is when $B^{\prime}$ is one irreducible component of $B$, in which case the lemma says that a locally free sheaf on $B$ which is generically ample in the sense of Lazarsfeld [Ful98] is deformation ample. 


\section{RATIONAL CURVES ON HYPERSURFACES, II}

\section{Deformation theory of stable maps}

The Kontsevich moduli space of genus-0 stable maps, $\overline{\mathcal{M}}_{0, r}(X, e)$, and the Behrend-Manin moduli spaces, $\overline{\mathcal{M}}(X, \tau)$, are described in $\S 1.1$. The deformation theory of stable maps has been worked out in [BF98] and [Beh97]. Many specific deformation-theoretic results follow easily from these papers and are known to the experts, but have not been written down. Some of these specific results are proved in this section. Although these results will only be applied to genus-0 stable maps in this paper, the same arguments work for stable maps of arbitrary genus; in this section only, stable maps and stable $A$-graphs are not necessarily assumed to be of genus 0 .

Let $X$ and $T$ be schemes.

Definition 3.1. A family of $r$-pointed, genus-g prestable maps to $X$ over $B$ is a triple

$$
\zeta=\left(\left(p: \Sigma \rightarrow B, \sigma_{1}, \ldots, \sigma_{r}\right), g: \Sigma \rightarrow X\right)
$$

consisting of a family $p: \Sigma \rightarrow B$ of prestable curves of genus $g$ over $B$, a sequence of $r$ disjoint sections $\sigma_{i}: B \rightarrow \Sigma$ with image contained in the smooth locus of $p$ (if $r=0$, the sections are omitted), and a morphism $g: \Sigma \rightarrow X$.

Let $\left(\left(\Sigma, \sigma_{1}, \ldots, \sigma_{r}\right), g\right)$ be a prestable map over an algebraically closed field. An irreducible component $\Sigma_{i} \subset \Sigma$ is stable if the restriction of the log-dualizing sheaf, $\mathcal{O}_{\Sigma}\left(K_{\Sigma}+\sigma_{1}+\cdots+\sigma_{r}\right) \otimes_{\mathcal{O}_{\Sigma}} \mathcal{O}_{\Sigma_{i}}$, is $g$-relatively ample, i.e. one of the following hold

i) $g: \Sigma_{i} \rightarrow X$ is nonconstant,

ii) $p_{a}\left(\Sigma_{i}\right)>1$,

iii) $p_{a}\left(\Sigma_{i}\right)=1$ and $\Sigma_{i}$ contains at least one marked point or external node of $\Sigma$, or

iv) $p_{a}\left(\Sigma_{i}\right)=0$ and $\Sigma_{i}$ contains at least three marked points and nodes of $B$.

The curve $\Sigma$ is stable if $\mathcal{O}_{\Sigma}\left(K_{\Sigma}+\sigma_{1}+\cdots+\sigma_{r}\right)$ is $g$-relatively ample, i.e. every irreducible component of $\Sigma$ is stable. The family $\zeta$ is stable if, for each geometric point $t \in T$, the curve $\Sigma_{t}$ is stable.

Now assume that $X$ is smooth.

Notation 3.2. Denote by $L_{\zeta}$ the complex of coherent sheaves on $\Sigma$

$$
\begin{array}{cc}
-1 & 0 \\
g^{*} \Omega_{X} \stackrel{(d g)^{\dagger}}{\longrightarrow} & \Omega_{p}\left(\sigma_{1}(T)+\cdots+\sigma_{r}(T)\right),
\end{array}
$$

where -1 or 0 indicates the degree.

For a scheme $T$ and a bounded-above complex of coherent sheaves $C$ on $T, C^{\vee}$ denotes the object in the derived category of coherent sheaves on $T$,

$$
C^{\vee}:=\mathbb{R H o m}_{\mathcal{O}_{T}}\left(C, \mathcal{O}_{T}\right) .
$$

In particular, $L_{\zeta}^{\vee}$ is the object

$$
L_{\zeta}^{\vee}=\mathbb{R H o m}_{\mathcal{O}_{\Sigma}}\left(L_{\zeta}, \mathcal{O}_{\Sigma}\right)
$$

in the derived category of coherent sheaves on $\Sigma$.

The relevance of the complex $L_{\zeta}^{\vee}$ is the following.

Lemma 3.3. Let $X$ be a smooth quasi-projective scheme. Let $\overline{\mathcal{M}}_{g, r}(X, \beta)$ denote the DeligneMumford stack of $r$-pointed stable maps to $X$ of arithmetic genus $g$ and degree $\beta$. Let $p: \Sigma \rightarrow$ $\overline{\mathcal{M}}_{g, r}(X, \beta)$ denote the universal curve, let $\sigma_{i}: \overline{\mathcal{M}}_{g, r}(X, \beta) \rightarrow \Sigma$ denote the universal sections, and let $g: \Sigma \rightarrow X$ denote the universal map, i.e.

$$
\zeta=\left(\left(p: \Sigma \rightarrow \overline{\mathcal{M}}_{g, r}(X, \beta), \sigma_{1}, \ldots, \sigma_{r}\right), g: \Sigma \rightarrow X\right)
$$




\section{J. HARRIS AND J. STARR}

is the universal family of stable maps. There is an obstruction theory for $\overline{\mathcal{M}}_{g, r}(X, \beta)$ in the sense of [BF98, Definition 4.4] of the form

$$
\left(\mathbb{R} p_{*}\left(L_{\zeta}^{\vee}\right)[1]\right)^{\vee} \longrightarrow L_{\overline{\mathcal{M}}_{g, r}(X, \beta)} \cdot
$$

A similar result holds for prestable maps; cf. Remark 3.4.

Proof. Essentially this follows from [BF98] and [Beh97].

Remark 3.4. Explicitly, if $\zeta=\left(\left(\Sigma, \sigma_{1}, \ldots, \sigma_{r}\right), g: \Sigma \rightarrow X\right)$ is a stable map or a prestable map, the space of first-order deformations of $\zeta$ is $\operatorname{Ext}_{\mathcal{O}_{\Sigma}}^{1}\left(L_{\zeta}, \mathcal{O}_{\Sigma}\right)$ and the obstruction group is a subgroup of $\mathbb{E x t}_{\mathcal{O}_{\Sigma}}^{2}\left(L_{\zeta}, \mathcal{O}_{\Sigma}\right)$. In the case of a prestable map, the space of infinitesimal automorphisms of the map is $\mathbb{E x t}_{\mathcal{O}_{\Sigma}}^{0}\left(L_{\zeta}, \mathcal{O}_{\Sigma}\right)$ (for stable maps this group is zero). In particular, if $\mathbb{E x t}_{\mathcal{O}_{\Sigma}}^{2}\left(L_{\zeta}, \mathcal{O}_{\Sigma}\right)$ vanishes, then $\overline{\mathcal{M}}_{g, r}(X, \beta)$ is smooth at the point $[\zeta]$.

Lemma 3.5. Let $\left(\left(\Sigma, \sigma_{1}, \ldots, \sigma_{r}\right), g\right)$ be a prestable map. Let $\Theta_{\Sigma}$ denote the tangent sheaf of $\Sigma$, i.e. the dual of $\Omega_{\Sigma}$. The space of infinitesimal automorphisms of $\zeta, \mathbb{E x t}_{\mathcal{O}_{\Sigma}}^{0}\left(L_{\zeta}, \mathcal{O}_{\Sigma}\right)$, is canonically isomorphic to a subspace of the space of infinitesimal automorphisms of $\left(\Sigma, \sigma_{1}, \ldots, \sigma_{r}\right)$, $H^{0}\left(\Sigma, \Theta_{\Sigma}\left(-\left(\sigma_{1}+\cdots+\sigma_{r}\right)\right)\right)$.

An irreducible component $\Sigma_{i} \subset \Sigma$ is stable if and only if the restriction of every infinitesimal automorphism of $\zeta$ to $\Theta_{\Sigma}\left(-\left(\sigma_{1}+\cdots+\sigma_{r}\right)\right) \otimes_{\mathcal{O}_{\Sigma}} \mathcal{O}_{\Sigma_{i}}$ is zero. Moreover, if $\Sigma_{i}$ is unstable, then every infinitesimal automorphism of the nodal curve $\Sigma_{i}$ that fixes all marked points and nodes of $\Sigma$ is the image of an infinitesimal automorphism of $\zeta$.

Proof. Analyzing the spectral sequence for hypercohomology, $\mathbb{E x t}_{\mathcal{O}_{\Sigma}}^{0}\left(L_{\zeta}, \mathcal{O}_{\Sigma}\right)$ is canonically isomorphic to the kernel of

$$
\operatorname{Ext}_{\mathcal{O}_{\Sigma}}^{0}\left(\Omega_{\Sigma}\left(\sigma_{1}+\cdots+\sigma_{r}\right), \mathcal{O}_{\Sigma}\right) \longrightarrow \operatorname{Ext}_{\mathcal{O}_{\Sigma}}^{0}\left(g^{*} \Omega_{X}, \mathcal{O}_{\Sigma}\right) .
$$

$\operatorname{Ext}_{\mathcal{O}_{\Sigma}}^{0}\left(\Omega_{\Sigma}\left(\sigma_{1}+\cdots+\sigma_{r}\right), \mathcal{O}_{\Sigma}\right)$ equals $H^{0}\left(\Sigma, \Theta_{\Sigma}\left(-\left(\sigma_{1}+\cdots+\sigma_{r}\right)\right)\right)$.

Assume that $\Sigma_{i}$ is stable, i.e. $\Sigma_{i}$ satisfies one of the cases i-iv in Definition 3.1. In cases ii-iv, the image of

$$
H^{0}\left(\Sigma, \Theta_{\Sigma}\left(-\left(\sigma_{1}+\cdots+\sigma_{r}\right)\right)\right) \longrightarrow H^{0}\left(\Sigma_{i}, \Theta_{\Sigma}\left(-\left(\sigma_{1}+\cdots+\sigma_{r}\right)\right) \otimes_{\mathcal{O}_{\Sigma}} \mathcal{O}_{\Sigma_{i}}\right)
$$

is zero hence the restriction of every infinitesimal automorphism of $\zeta$ is zero. In case i, chasing through diagrams, the image of $H^{0}\left(\Sigma, \Theta_{\Sigma}\left(-\left(\sigma_{1}+\cdots+\sigma_{r}\right)\right)\right)$ is contained in the subsheaf which is the kernel of $d\left(\left.g\right|_{\Sigma_{i}}\right): \Theta_{\Sigma_{i}} \rightarrow g^{*} T_{X} \otimes_{\mathcal{O}_{\Sigma}} \mathcal{O}_{\Sigma_{i}}$. Since $g$ is nonconstant, this map is nonzero. $\Theta_{\Sigma_{i}}$ is a torsion-free sheaf, so the kernel of $d\left(\left.g\right|_{\Sigma_{i}}\right)$ is zero. Hence, also in case i, the restriction of every infinitesimal automorphism of $\zeta$ is zero.

Assume that $\Sigma_{i}$ is not stable. Then $g$ contracts $\Sigma_{i}$ to a point, and either $\Sigma=\Sigma_{i}$ is a curve of arithmetic genus 1 and there are no marked points, or $\Sigma_{i}$ is a smooth rational curve which contains at most two marked points and nodes of $\Sigma$. In both cases there is a positive dimensional group of automorphisms of $\Sigma_{i}$ which fix all marked points and nodes of $\Sigma$ : $\operatorname{Pic}^{\tau}\left(\Sigma_{i}\right)$ acting by translation if $\Sigma_{i}$ has arithmetic genus 1 , and the group of automorphisms of $\mathbb{P}^{1}$ fixing (at most) two points if $\Sigma_{i}$ is a smooth rational curve. The Lie algebra of this positive dimensional group is the Lie algebra of infinitesimal automorphisms of the marked curve $\Sigma_{i}$. This group of automorphisms of $\Sigma_{i}$ extends to a group of automorphisms of the map $\zeta$. Therefore every infinitesimal automorphism of the marked curve $\Sigma_{i}$ extends to an infinitesimal automorphism of $\zeta$. In particular there exists an infinitesimal automorphism of $\zeta$ whose restriction to $\Sigma_{i}$ is nonzero.

Let $f: X \rightarrow Y$ be a smooth morphism of smooth quasi-projective varieties. Let $\zeta=\left(\left(\Sigma, \sigma_{1}, \ldots, \sigma_{r}\right), g: \Sigma \rightarrow X\right)$ be a prestable map. Denote by $h: \Sigma \rightarrow Y$ the composition $h=f \circ g$, 
and denote by $\xi$ the prestable map $\left(\left(\Sigma, \sigma_{1}, \ldots, \sigma_{r}\right), h: \Sigma \rightarrow Y\right)$. There is a short exact sequence of complexes

$$
0 \longrightarrow L_{\xi} \longrightarrow L_{\zeta} \longrightarrow g^{*} \Omega_{f}[1] \longrightarrow 0
$$

defined by the following commutative diagram:

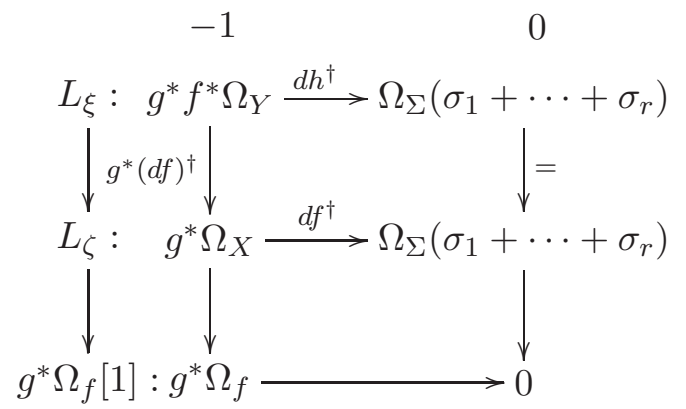

LEMMA 3.6.

i) If $\xi$ is stable, then $\zeta$ is stable.

ii) If the dimension of the obstruction group of $\zeta$ is 0 , then the dimension of the obstruction group of $\xi$ is 0 .

iii) If $h^{1}\left(\Sigma, g^{*} \Omega_{f}^{\vee}\right)=0$ and if the dimension of the obstruction group of $\xi$ is 0 , then the dimension of the obstruction group of $\zeta$ is 0 , and the map from the space of first-order deformations of $\zeta$ to the space of first-order deformations of $\xi$ is surjective.

Proof. i) If the log dualizing sheaf of $\left(\Sigma, \sigma_{1}, \ldots, \sigma_{r}\right)$ is $h$-ample, then it is $g$-ample.

ii) Associated to the short exact sequence of complexes above, there is a long exact sequence of hyper Ext, part of which is

$$
\mathbb{E x t}_{\mathcal{O}_{\Sigma}}^{2}\left(L_{\zeta}, \mathcal{O}_{\Sigma}\right) \longrightarrow \mathbb{E x t}_{\mathcal{O}_{\Sigma}}^{2}\left(L_{\xi}, \mathcal{O}_{\Sigma}\right) \longrightarrow \mathbb{E x t}_{\mathcal{O}_{\Sigma}}^{3}\left(g * \Omega_{f}[1], \mathcal{O}_{\Sigma}\right)
$$

Of course $\quad \operatorname{Ext}_{\mathcal{O}_{\Sigma}}^{k}\left(g^{*} \Omega_{f}[1], \mathcal{O}_{\Sigma}\right)=H^{k-1}\left(\Sigma, g^{*} \Omega_{f}^{\vee}\right)$. In particular $\operatorname{dim} \mathbb{E x t}_{\mathcal{O}_{\Sigma}}^{3}\left(g^{*} \Omega_{f}[1], \mathcal{O}_{\Sigma}\right)=$ $h^{2}\left(\Sigma, g^{*} \Omega_{f}^{\vee}\right)=0$. Therefore if $\operatorname{dim} \mathbb{E x t}_{\mathcal{O}_{\Sigma}}^{2}\left(L_{\zeta}, \mathcal{O}_{\Sigma}\right)=0$, then $\operatorname{dim} \mathbb{E x t}_{\mathcal{O}_{\Sigma}}^{2}\left(L_{\xi}, \mathcal{O}_{\Sigma}\right)=0$.

iii) If $h^{1}\left(\Sigma, g^{*} \Omega_{f}^{\vee}\right)=0$, then $\mathbb{E x t}_{\mathcal{O}_{\Sigma}}^{2}\left(L_{\zeta}, \mathcal{O}_{\Sigma}\right) \rightarrow \mathbb{E x t}_{\mathcal{O}_{\Sigma}}^{2}\left(L_{\xi}, \mathcal{O}_{\Sigma}\right)$ is an isomorphism. Hence if the dimension of the obstruction group of $\zeta$ is 0 , then the dimension of the obstruction group of $\xi$ is 0 . Moreover the preceding two terms in the long exact sequence of hyper Ext give a surjection $\operatorname{Ext}_{\mathcal{O}_{\Sigma}}^{1}\left(L_{\zeta}, \mathcal{O}_{\Sigma}\right) \rightarrow \mathbb{E x t}_{\mathcal{O}_{\Sigma}}^{1}\left(L_{\xi}, \mathcal{O}_{\Sigma}\right)$.

Let $q: S \rightarrow B$ be a smooth morphism and let $i: \Sigma \rightarrow S$ be an unramified morphism of $B$-schemes. Then $(g, i): \Sigma \rightarrow X \times S$ is an unramified morphism of $B$-schemes; hence the map of coherent sheaves

$$
\left.d(g, i)^{\dagger}:(g, i)^{*} \Omega_{(} X \times S / B\right) \longrightarrow \Omega_{p}
$$

is surjective. Because $p$ is flat of relative dimension 1 and the geometric fibers are reduced, local complete intersection schemes, the kernel of $d(g, i)^{\dagger}$ is a locally free sheaf $N_{(g, i)}^{\vee}$. Similarly the kernel of $d i^{\dagger}: i^{*} \Omega_{q} \rightarrow \Omega_{p}$ is a locally free sheaf $N_{i}^{\vee}$.

There is a short exact sequence of coherent sheaves,

$$
0 \longrightarrow N_{i}^{\vee} \longrightarrow N_{(g, i)}^{\vee} \longrightarrow g^{*} \Omega_{X} \longrightarrow 0 .
$$

Denote by $N_{(\zeta, i)}^{\vee}$ the subsheaf of $N_{(g, i)}^{\vee}\left(\sigma_{1}(B)+\cdots+\sigma_{r}(B)\right)$ that contains $N_{i}^{\vee}\left(\sigma_{1}(B)+\cdots+\sigma_{r}(B)\right)$ and such that $N_{(\zeta, i)}^{\vee} / N_{i}^{\vee}\left(\sigma_{1}(B)+\cdots+\sigma_{r}(B)\right)$ is identified with $g^{*} \Omega_{X} \subset g^{*} \Omega_{X}\left(\sigma_{1}(B)+\cdots+\sigma_{r}(B)\right)$. Observe that $N_{(\zeta, i)}^{\vee}$ is a locally free sheaf. 


\section{J. HARRIS AND J. STARR}

Notation 3.7. There is a canonical map

$$
N_{(g, i)}^{\vee}\left(\sigma_{1}(B)+\cdots+\sigma_{r}(B)\right) \longrightarrow(g, i)^{*} \Omega_{X \times S / B}
$$

The two projections give a canonical isomorphism of $\Omega_{X \times S / B}$ with $\pi_{X}^{*} \Omega_{X} \oplus \pi_{S}^{*} \Omega_{q}$. Denote by

$$
\gamma_{(\zeta, i)}: N_{(\zeta, i)}^{\vee} \longrightarrow g^{*} \Omega_{X}\left(\sigma_{1}(B)+\cdots+\sigma_{r}(B)\right) \oplus i^{*} \Omega_{q}\left(\sigma_{1}(B)+\cdots+\sigma_{r}(B)\right)
$$

the induced morphism. Observe that the composition of $\gamma_{(\zeta, i)}$ with projection on the first summand factors through

$$
g^{*} \Omega_{X} \subset g^{*} \Omega_{X}\left(\sigma_{1}(B)+\cdots+\sigma_{r}(B)\right) .
$$

Denote by $\alpha_{(\zeta, i)}: N_{(\zeta, i)}^{\vee} \rightarrow g^{*} \Omega_{X}$ and $\beta_{(\zeta, i)}: N_{(\zeta, i)}^{\vee} \rightarrow i^{*} \Omega_{q}\left(\sigma_{1}(B)+\cdots+\sigma_{r}(B)\right)$ the composition of $\gamma_{(\zeta, i)}$ with the two projections. Denote by $L_{(\zeta, i)}$ the complex of locally free $\mathcal{O}_{\Sigma}$-modules concentrated in degrees $[-1,0]$ :

$$
\begin{array}{cc}
-1 & 0 \\
N_{(\zeta, i)}^{\vee} \stackrel{\beta_{(\zeta, i)}}{\longrightarrow} i^{*} \Omega_{q}\left(\sigma_{1}(B)+\cdots+\sigma_{r}(B)\right)
\end{array}
$$

Denote by $\lambda_{(\zeta, i)}: L_{(\zeta, i)} \rightarrow L_{\zeta}$ the quasi-isomorphism of complexes of coherent $\mathcal{O}_{\Sigma}$-modules

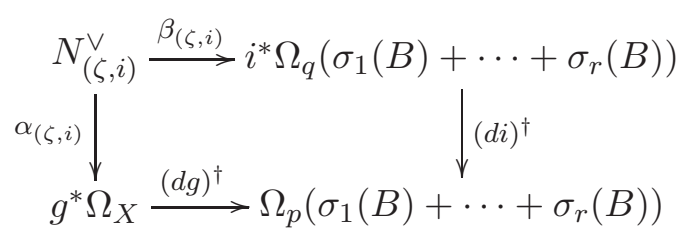

The relevance of $\lambda_{(\zeta, i)}: L_{(\zeta, i)} \rightarrow L_{\zeta}$ is that the complex $L_{(\zeta, i)}^{\vee}$ is easy to compute since $L_{(\zeta, i)}$ is a complex of locally free sheaves; it is simply

$$
\begin{gathered}
0 \\
i^{*} T_{q}\left(-\left(\sigma_{1}(B)+\cdots+\sigma_{r}(B)\right)\right) \stackrel{\beta_{(\zeta, i)}^{\dagger}}{\longrightarrow} N_{(\zeta, i)} .
\end{gathered}
$$

In most applications, $B$ will be the spectrum of a field and $S$ will be a surface.

\subsection{Contracting unstable components}

In this section, the base $B$ will always be the spectrum of an algebraically closed field. The changes necessary to get relative versions of the lemmas over a more general base are straightforward.

Let

$$
\zeta=\left(\left(\Sigma, \sigma_{1}, \ldots, \sigma_{r}\right), g: \Sigma \rightarrow X\right)
$$

be a prestable map, let

$$
\left(\Sigma^{\prime}, \sigma_{1}^{\prime}, \ldots, \sigma_{r}^{\prime}, \sigma_{1}^{\prime \prime}, \ldots, \sigma_{s}^{\prime \prime}\right)
$$

be a proper, connected, at-worst-nodal curve, and let

$$
u:\left(\Sigma^{\prime}, \sigma_{1}^{\prime}, \ldots, \sigma_{r}^{\prime}\right) \longrightarrow\left(\Sigma, \sigma_{1}, \ldots, \sigma_{r}\right)
$$

be a map which contracts some of the unstable components of $\left(\Sigma^{\prime}, \sigma_{1}^{\prime}, \ldots, \sigma_{r}^{\prime}\right)$ (i.e. $u$ contracts some of the irreducible components of $\Sigma^{\prime}$ which have arithmetic genus 0 and contain fewer than three nodes and marked points). Denote $g^{\prime}=g \circ u$ and denote by $\zeta^{\prime}$ the prestable map

$$
\zeta^{\prime}=\left(\left(\Sigma^{\prime}, \sigma_{1}^{\prime}, \ldots, \sigma_{r}^{\prime}, \sigma_{1}^{\prime \prime}, \ldots, \sigma_{s}^{\prime \prime}\right), g^{\prime}: \Sigma^{\prime} \rightarrow X\right) .
$$

What is the relationship of $\operatorname{Ext}_{\mathcal{O}_{\Sigma}}\left(L_{\zeta}, \mathcal{O}_{\Sigma}\right)$ and $\mathbb{E x t}_{\mathcal{O}_{\Sigma^{\prime}}}\left(L_{\zeta^{\prime}}, \mathcal{O}_{\Sigma^{\prime}}\right)$ ? 


\section{RATiONAL CURVES ON HYPERSURFACES, II}

Any morphism $u: \Sigma^{\prime} \rightarrow \Sigma$ as above can be factored as a sequence of elementary morphisms, defined below.

\section{DEFINITION 3.8.}

i) The morphism $u$ is a Type I elementary morphism if $\zeta=(\Sigma, g: \Sigma \rightarrow X)$ is a prestable map without marked points, $u: \Sigma^{\prime} \rightarrow \Sigma$ contracts a single unstable component to a smooth point of $\Sigma$, and $\zeta^{\prime}=\left(\Sigma^{\prime}, g^{\prime}=g \circ u: \Sigma^{\prime} \rightarrow X\right)$.

ii) The morphism $u$ is a Type II elementary morphism if $\zeta=(\Sigma, g: \Sigma \rightarrow X)$ is a prestable map without marked points, $u: \Sigma^{\prime} \rightarrow \Sigma$ contracts a single unstable component to a node of $\Sigma$, and $\zeta^{\prime}=\left(\Sigma^{\prime}, g^{\prime}=g \circ u: \Sigma^{\prime} \rightarrow X\right)$.

iii) The morphism $u$ is a Type III elementary morphism if $\zeta=\left(\Sigma,\left(\sigma_{1}, \ldots, \sigma_{r}\right), g: \Sigma \rightarrow X\right)$ is a marked prestable map, $\zeta^{\prime}$ is the same prestable map but with one extra marked point, and $u: \Sigma^{\prime} \rightarrow \Sigma$ is the identity map.

Let $S$ be a smooth surface and let $i: \Sigma \rightarrow S$ be a closed immersion. Let $s \in \Sigma \subset S$ be a closed point.

Notation 3.9. Denote by $v: S^{\prime} \rightarrow S$ the blowing-up of $S$ at $s$. Denote by $E \subset S$ the exceptional divisor. Denote by $i^{\prime}: \Sigma^{\prime} \rightarrow S^{\prime}$ the reduced total transform of $B$, i.e. the reduced scheme of $v^{-1}(\Sigma)$. Denote by $v$ also the morphism of pairs $v:\left(S^{\prime}, \Sigma^{\prime}\right) \rightarrow(S, \Sigma)$. Denote by $u: \Sigma^{\prime} \rightarrow \Sigma$ the restriction of $v$ to $\Sigma^{\prime}$. Denote by $g^{\prime}: \Sigma^{\prime} \rightarrow X$ the composition $g^{\prime}=g \circ u$. Denote by $\zeta^{\prime}$ the prestable map $\left(\Sigma^{\prime}, g^{\prime}: \Sigma^{\prime} \rightarrow X\right)$. Denote by $\Gamma \subset \Sigma^{\prime}$ the closed (not necessarily connected) subcurve which is the union of all irreducible components other than $E$, and denote $D=E \cap \Gamma$.

\section{DEFINITION 3.10.}

i) The morphism of pairs is Type $I a$ if $s \in \Sigma$ is a smooth point that lies on a stable component.

ii) The morphism of pairs is Type $I b$ if $s \in \Sigma$ is a smooth point that lies on an unstable component.

iii) The morphism of pairs is Type $I$ if it is Type Ia or Type Ib.

iv) The morphism of pairs is Type IIa if $s \in \Sigma$ is a node, and there exists a first-order deformation of $\zeta$ that smoothes the node $s$ (to first order).

v) The morphism of pairs is Type IIb if $s \in \Sigma$ is a node, and there is no first-order deformation of $\zeta$ that smoothes the node $s$ (to first order).

vi) The morphism of pairs is Type II if it is Type IIa or Type IIb.

If $v$ is Type I, then $v^{*} \Sigma=\Sigma^{\prime}$ as Cartier divisors. If $v$ is Type II, then $v^{*} \Sigma=\Sigma^{\prime}+E$ as Cartier divisors.

Lemma 3.11. For every integer $k$ the pullback morphism of sheaf hypercohomology groups, $\mathbb{H}^{k}\left(\Sigma, L_{\zeta}^{\vee}\right) \rightarrow \mathbb{H}^{k}\left(\Sigma^{\prime}, \mathbb{L} u^{*} L_{\zeta}^{\vee}\right)$, is an isomorphism.

Proof. Of course $v_{*} \mathcal{O}_{S^{\prime}}=\mathcal{O}_{S}$ and $R^{k} v_{*} \mathcal{O}_{S^{\prime}}=\{0\}$ if $k>0$. Also $v_{*}\left(\mathcal{O}_{S^{\prime}}(E)\right)=\mathcal{O}_{S}$ and $R^{k} v_{*}\left(\mathcal{O}_{S^{\prime}}(E)\right)=\{0\}$ for $k>0$. If $v$ is Type I then $\mathcal{O}_{S^{\prime}}\left(-\Sigma^{\prime}\right)=v^{*} \mathcal{O}_{S}(-\Sigma)$, and if $v$ is Type II then $\mathcal{O}_{S^{\prime}}\left(-\Sigma^{\prime}\right)=v^{*} \mathcal{O}_{S}(-\Sigma) \otimes_{\mathcal{O}_{S^{\prime}}} \mathcal{O}_{S^{\prime}}(E)$. For both types, the projection formula implies that $v_{*}\left(\mathcal{O}_{S^{\prime}}\left(-\Sigma^{\prime}\right)\right)=\mathcal{O}_{S}(-\Sigma)$ and $R^{k} v_{*}\left(\mathcal{O}_{S^{\prime}}\left(-\Sigma^{\prime}\right)\right)=\{0\}$ for $k>0$.

Associated to the short exact sequence of coherent $\mathcal{O}_{S^{\prime}}$-modules,

$$
0 \longrightarrow \mathcal{O}_{S^{\prime}}\left(-\Sigma^{\prime}\right) \longrightarrow \mathcal{O}_{S^{\prime}} \longrightarrow \mathcal{O}_{\Sigma^{\prime}} \longrightarrow 0,
$$

there is a long exact sequence of higher direct images $R^{k} v_{*}$, and the higher direct images of $\mathcal{O}_{S^{\prime}}$ and $\mathcal{O}_{S^{\prime}}\left(-\Sigma^{\prime}\right)$ have just been computed. The conclusion is that $u_{*} \mathcal{O}_{\Sigma^{\prime}}=\mathcal{O}_{\Sigma}$ and $R^{k} u_{*} \mathcal{O}_{\Sigma^{\prime}}=\{0\}$ 


\section{J. HARRIS AND J. STARR}

for $k>0$. In other words, the canonical morphism of complexes of coherent $\mathcal{O}_{\Sigma}$-modules, $\mathcal{O}_{\Sigma}[0] \rightarrow$ $\mathbb{R} u_{*} \mathcal{O}_{\Sigma^{\prime}}$, is a quasi-isomorphism. From this and the projection formula, it follows that the canonical morphism

$$
L_{(\zeta, i)}^{\vee} \longrightarrow \mathbb{R} u_{*} \mathbb{L} u^{*}\left(L_{(\zeta, i)}^{\vee}\right)
$$

is a quasi-isomorphism. Therefore the pullback morphisms

$$
\mathbb{H}^{k}\left(\Sigma, L_{\zeta}^{\vee}\right) \longrightarrow \mathbb{H}^{k}\left(\Sigma^{\prime}, \mathbb{L} u^{*} L_{\zeta}^{\vee}\right)
$$

are isomorphisms.

There is a canonical map of coherent sheaves $\mathcal{O}_{S^{\prime}}\left(\Sigma^{\prime}\right) \rightarrow v^{*} \mathcal{O}_{S}(\Sigma)$. It is an isomorphism if $v$ is Type I, and is injective with cokernel $v^{*} \mathcal{O}_{S}(\Sigma) \otimes_{\mathcal{O}_{S^{\prime}}} \mathcal{O}_{E}$ if $v$ is Type II. If $v$ is Type II, then $v^{*} \mathcal{O}_{X}(\Sigma) \otimes_{\mathcal{O}_{S^{\prime}}} \mathcal{O}_{E} \cong M \otimes_{\mathbb{C}} \mathcal{O}_{E}$ where $M=\left.\mathcal{O}_{S}(\Sigma)\right|_{s}$ is a one-dimensional vector space. If $v$ is Type I, the canonical morphism $N_{\left(\zeta^{\prime}, i^{\prime}\right)} \rightarrow u^{*} N_{(\zeta, i)}$ is an isomorphism. If $v$ is Type II, there is an exact sequence:

$$
0 \longrightarrow M \otimes_{\mathbb{C}} \operatorname{Tor}_{1}^{\mathcal{O}_{S^{\prime}}}\left(\mathcal{O}_{\Sigma^{\prime}}, \mathcal{O}_{E}\right) \longrightarrow N_{\left(\zeta^{\prime}, i^{\prime}\right)} \longrightarrow u^{*} N_{(\zeta, i)} \longrightarrow M \otimes_{\mathbb{C}} \mathcal{O}_{E} \longrightarrow 0 .
$$

Lemma 3.12. Let $N_{E / S^{\prime}}^{\vee}$ denote the conormal sheaf of $E \subset S^{\prime}$. There is a canonical isomorphism $\operatorname{Tor}_{1}^{\mathcal{O}_{S^{\prime}}}\left(\mathcal{O}_{\Sigma^{\prime}}, \mathcal{O}_{E}\right) \cong N_{E / S^{\prime}}^{\vee} \otimes \mathcal{O}_{E} \mathcal{O}_{E}(-D)$.

Proof. There is an $\mathcal{O}_{S^{\prime}}$-flat resolution of $\mathcal{O}_{E}$,

$$
0 \longrightarrow \mathcal{O}_{S^{\prime}}(-E) \longrightarrow \mathcal{O}_{S} \longrightarrow \mathcal{O}_{E} \longrightarrow 0
$$

Tensoring this resolution with $\mathcal{O}_{\Sigma^{\prime}}$ over $\mathcal{O}_{S^{\prime}}$ gives a canonical isomorphism,

$$
\operatorname{Tor}_{1}^{\mathcal{O}_{S^{\prime}}}\left(\mathcal{O}_{\Sigma^{\prime}}, \mathcal{O}_{E}\right) \cong \mathcal{I}_{\Gamma} \otimes_{\mathcal{O}_{S^{\prime}}} \mathcal{O}_{S^{\prime}}(-E) \cong N_{E / S^{\prime}}^{\vee} \otimes_{\mathcal{O}_{E}} \mathcal{O}_{E}(-D)
$$

where $\mathcal{I}_{\Gamma} \subset \mathcal{O}_{\Sigma^{\prime}}$ is the ideal sheaf of $\Gamma \subset \Sigma^{\prime}$.

In particular, if $v$ is Type II there is an exact sequence:

$$
0 \longrightarrow M \otimes_{\mathbb{C}} N_{E / S^{\prime}}^{\vee} \otimes_{\mathcal{O}_{E}} \mathcal{O}_{E}(-D) \longrightarrow N_{\left(\zeta^{\prime}, i^{\prime}\right)} \longrightarrow u^{*} N_{(\zeta, i)} \longrightarrow M \otimes_{\mathbb{C}} \mathcal{O}_{E} \longrightarrow 0 .
$$

Lemma 3.13. There is a long exact sequence,

$$
0 \longrightarrow T_{E}(-D) \longrightarrow\left(i^{\prime}\right)^{*} T_{S^{\prime}} \stackrel{d v}{\longrightarrow} u^{*} i^{*} T_{S} \longrightarrow T_{E} \otimes_{\mathcal{O}_{E}} N_{E / S^{\prime}} \longrightarrow 0 .
$$

(Both if $v$ is Type $I$ and if $v$ is Type II.)

Proof. For both types, there is a short exact sequence of coherent $\mathcal{O}_{S^{\prime}}$-modules,

$$
0 \longrightarrow v^{*} \Omega_{S} \stackrel{(d v)^{\dagger}}{\longrightarrow} \Omega_{S^{\prime}} \longrightarrow \Omega_{E} \longrightarrow 0 .
$$

There is an associated long exact sequence of higher derived functors of $\operatorname{Hom}_{\mathcal{O}_{S^{\prime}}}\left(\cdot, \mathcal{O}_{S^{\prime}}\right)$, part of which is the short exact sequence,

$$
0 \longrightarrow T_{S^{\prime}} \longrightarrow v^{*} T_{S} \longrightarrow \operatorname{Ext}_{\mathcal{O}_{S^{\prime}}}^{1}\left(\Omega_{E}, \mathcal{O}_{S^{\prime}}\right) \longrightarrow 0
$$

The resolution of $\mathcal{O}_{E}$ from the proof of Lemma 3.12 gives a canonical isomorphism,

$$
\operatorname{Ext}_{\mathcal{O}_{S^{\prime}}}^{1}\left(\mathcal{O}_{E}, \mathcal{O}_{S^{\prime}}\right) \cong N_{E / S^{\prime}}
$$

where $N_{E / S^{\prime}}$ is the dual of $N_{E / S^{\prime}}^{\vee}$. So the previous exact sequence is

$$
0 \longrightarrow T_{S^{\prime}} \longrightarrow v^{*} T_{S} \longrightarrow T_{E} \otimes_{\mathcal{O}_{E}} N_{E / S^{\prime}} \longrightarrow 0 .
$$




\section{RATIONAL CURVES ON HYPERSURFACES, II}

Of course $T_{E} \otimes_{\mathcal{O}_{E}} N_{E / S^{\prime}}$ is a locally free $\mathcal{O}_{E}$-module; in particular, we have a canonical isomorphism,

$$
\operatorname{Tor}_{1}^{\mathcal{O}_{S^{\prime}}}\left(\mathcal{O}_{\Sigma^{\prime}}, T_{E} \otimes_{\mathcal{O}_{E}} N_{E / S^{\prime}}\right) \cong \operatorname{Tor}_{1}^{\mathcal{O}_{S^{\prime}}}\left(\mathcal{O}_{\Sigma^{\prime}}, \mathcal{O}_{E}\right) \otimes_{\mathcal{O}_{E}} T_{E} \otimes_{\mathcal{O}_{E}} N_{E / S^{\prime}}
$$

Tensoring the short exact sequence above with $\mathcal{O}_{\Sigma^{\prime}}$ over $\mathcal{O}_{S^{\prime}}$ and using Lemma 3.12 produces the exact sequence,

$$
0 \longrightarrow T_{E}(-D) \longrightarrow\left(i^{\prime}\right)^{*} T_{S^{\prime}} \stackrel{d v}{\longrightarrow} u^{*} i^{*} T_{S} \longrightarrow T_{E} \otimes_{\mathcal{O}_{E}} N_{E / S^{\prime}} \longrightarrow 0 .
$$

The maps $N_{\left(\zeta^{\prime}, i^{\prime}\right)} \rightarrow u^{*} N_{(\zeta, i)}$ and $\left(i^{\prime}\right)^{*} T_{S^{\prime}} \rightarrow u^{*} i^{*} T_{S}$ are compatible with $\alpha_{\left(\zeta^{\prime}, i^{\prime}\right)}^{\dagger}$ and $u^{*} \alpha_{(\zeta, i)}^{\dagger}$. So there is an induced map of complexes $L_{\left(\zeta^{\prime}, i^{\prime}\right)}^{\vee} \rightarrow u^{*} L_{(\zeta, i)}^{\vee}$.

Notation 3.14 .

i) Denote by $d u: L_{\left(\zeta^{\prime}, i^{\prime}\right)}^{\vee} \rightarrow h^{*} L_{(\zeta, i)}^{\vee}$ the induced map of complexes.

ii) Denote by Image $(d u) \hookrightarrow u^{*} L_{(\zeta, i)}^{\vee}$ the image of $d u$ in the Abelian category of complexes of coherent $\mathcal{O}_{\Sigma^{\prime}}$-modules.

iii) If $v$ is Type I, denote $K_{\mathrm{I}}=T_{E}(-D)[0]$ and $Q_{\mathrm{I}}=\left(T_{E} \otimes_{\mathcal{O}_{E}} N_{E / S^{\prime}}\right)[0]$.

iv) If $v$ is Type II, denote

$$
\begin{aligned}
& K_{\mathrm{II}}=T_{E}(-D)[0] \oplus\left(M \otimes_{\mathbb{C}} N_{E / S^{\prime}}^{\vee}(-D)\right)[-1], \\
& Q_{\mathrm{II}}=\left(T_{E} \otimes_{\mathcal{O}_{E}} N_{E / S^{\prime}}\right)[0] \oplus\left(M \otimes_{\mathbb{C}} \mathcal{O}_{E}\right)[-1] .
\end{aligned}
$$

Lemma 3.15. Both if $v$ is Type $I$ and if $v$ is Type II, there are short exact sequences of complexes of coherent $\mathcal{O}_{\Sigma^{\prime}-\text { modules, }}$

$$
\begin{aligned}
& 0 \longrightarrow K \longrightarrow L_{\left(\zeta^{\prime}, i^{\prime}\right)}^{\vee} \stackrel{d u}{\longrightarrow} \operatorname{Image}(d u) \longrightarrow 0, \\
& 0 \longrightarrow \text { Image }(d u) \longrightarrow u^{*} L_{\zeta}^{\vee} \longrightarrow 0 \longrightarrow
\end{aligned}
$$

Proof. If $v$ is Type I, there is a commutative diagram with exact rows,

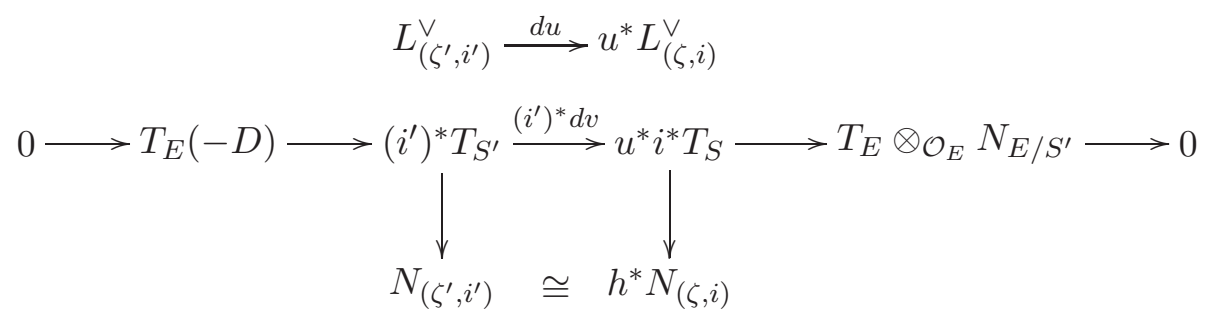

The middle two columns of this diagram give $d u: L_{\left(\zeta^{\prime}, i^{\prime}\right)}^{\vee} \rightarrow u^{*} L_{(\zeta, i)}^{\vee}$. By inspection the kernel of $d u$ is $K_{\mathrm{I}}$ and the cokernel of $d u$ is $Q_{\mathrm{I}}$.

If $v$ is Type II, there is a commutative diagram with exact rows,

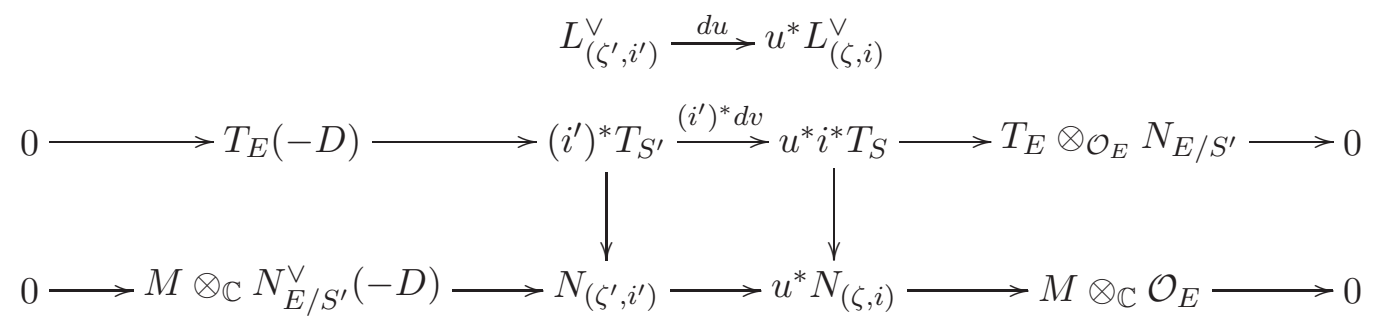

The middle two columns of this diagram give $d u: L_{\left(\zeta^{\prime}, i^{\prime}\right)}^{\vee} \rightarrow u^{*} L_{(\zeta, i)}^{\vee}$. 


\section{J. HARRIS AND J. STARR}

The claim is that the induced map of kernels, $T_{E}(-D) \rightarrow M \otimes_{\mathbb{C}} N_{E / S^{\prime}}^{\vee}(-D)$, is zero, and the induced map of cokernels, $T_{E} \otimes_{\mathcal{O}_{E}} N_{E / S^{\prime}} \rightarrow M \otimes_{\mathbb{C}} \mathcal{O}_{E}$, is zero. To see this, observe that in each map, the domain and target are locally free sheaves on $E$. So each map is really a section of a $\operatorname{Hom}_{\mathcal{O}_{E}}(\cdot, \cdot)$ sheaf. Up to canonical isomorphisms, the sheaf in both cases is simply

$$
\operatorname{Hom}_{\mathcal{O}_{E}}\left(T_{E}, M \otimes_{\mathbb{C}} N_{E / S^{\prime}}^{\vee}\right) \cong M \otimes_{\mathbb{C}} \Omega_{S^{\prime}}^{2} \otimes_{\mathcal{O}_{S}} \mathcal{O}_{E}
$$

Identifying $E$ with $\mathbb{P}^{1}$, there are isomorphisms $N_{E / S^{\prime}} \cong \mathcal{O}_{E}(-1)$ and $\mathcal{O}_{E}\left(K_{E}\right) \cong \mathcal{O}_{E}(-2)$. By the adjunction formula for divisors on surfaces, $\Omega_{S^{\prime}}^{2} \otimes_{\mathcal{O}_{S}} \mathcal{O}_{E}=\mathcal{O}_{S^{\prime}}\left(K_{S^{\prime}}\right) \otimes_{\mathcal{O}_{S}} \mathcal{O}_{E}$ is isomorphic to $\mathcal{O}_{E}\left(K_{E}\right) \otimes \mathcal{O}_{E} N_{E / S^{\prime}}^{\vee}$, i.e. $\Omega_{S^{\prime}}^{2} \otimes \mathcal{O}_{S} \mathcal{O}_{E} \cong \mathcal{O}_{E}(-2+1)=\mathcal{O}_{E}(-1)$. Since this sheaf has no nonzero global sections, both the induced maps are zero. Therefore the kernel of $d u$ is $K_{\text {II }}$ and cokernel of $d u$ is $Q_{\mathrm{II}}$.

Assume first that $v$ is Type I. Since $T_{E}(-D) \cong \mathcal{O}_{E}(1), \mathbb{H}^{0}\left(\Sigma^{\prime}, K_{\mathrm{I}}\right)=H^{0}\left(E, T_{E}(-D)\right)$ is twodimensional, and $\operatorname{dim} \mathbb{H}^{i}\left(\Sigma^{\prime}, K_{\mathrm{I}}\right)=0$ for $i \neq 0$. Similarly $\mathbb{H}^{0}\left(\Sigma^{\prime}, Q_{\mathrm{I}}\right)=H^{0}\left(E, T_{E}(E)\right)$ is two-dimensional and $\operatorname{dim} \mathbb{H}^{i}\left(\Sigma^{\prime}, Q_{\mathrm{I}}\right)=0$ for $i>0$. Therefore there is a long exact sequence of hypercohomology groups:

$$
\begin{aligned}
& 0 \rightarrow H^{0}\left(E, T_{E}(-D)\right) \rightarrow \mathbb{H}^{0}\left(\Sigma^{\prime}, L_{\left(\zeta^{\prime}, i^{\prime}\right)}^{\vee}\right) \\
& \cdots \rightarrow \mathbb{H}^{0}\left(\Sigma, L_{(\zeta, i)}^{\vee}\right) \rightarrow \cdots \\
& 0\left(E, T_{E} \otimes \mathcal{O}_{E} N_{E / S^{\prime}}\right) \rightarrow \mathbb{H}^{1}\left(\Sigma^{\prime}, L_{\left(\zeta^{\prime}, i^{\prime}\right)}^{\vee}\right) \\
& 0 \rightarrow \mathbb{H}^{1}\left(\Sigma, L_{(\zeta, i)}^{\vee}\right) \rightarrow 0, \\
& \mathbb{H}^{2}\left(\Sigma^{\prime}, L_{\left(\zeta^{\prime}, i^{\prime}\right)}^{\vee}\right) \rightarrow \mathbb{H}^{2}\left(\Sigma, L_{(\zeta, i)}^{\vee}\right) \rightarrow 0 .
\end{aligned}
$$

Lemma 3.16. If $v$ is Type Ia then there are exact sequences,

$$
\begin{aligned}
& 0 \rightarrow H^{0}\left(E, T_{E}(-D)\right) \rightarrow \mathbb{H}^{0}\left(\Sigma^{\prime}, L_{\left(\zeta^{\prime}, i^{\prime}\right)}^{\vee}\right) \rightarrow \mathbb{H}^{0}\left(\Sigma, L_{(\zeta, i)}^{\vee}\right) \rightarrow 0, \\
& 0 \rightarrow H^{0}\left(E, T_{E} \otimes \mathcal{O}_{E} N_{E / S^{\prime}}\right) \rightarrow \mathbb{H}^{1}\left(\Sigma^{\prime}, L_{\left(\zeta^{\prime}, i^{\prime}\right)}^{\vee}\right) \rightarrow \mathbb{H}^{1}\left(\Sigma, L_{(\zeta, i)}^{\vee}\right) \rightarrow 0, \\
& 0 \rightarrow \mathbb{H}^{2}\left(\Sigma^{\prime}, L_{\left(\zeta^{\prime}, i^{\prime}\right)}^{\vee}\right) \rightarrow \mathbb{H}^{2}\left(\Sigma, L_{(\zeta, i)}^{\vee}\right) \rightarrow 0 .
\end{aligned}
$$

In other words, the canonical map from the space of infinitesimal automorphisms of $\zeta^{\prime}$ to the space of infinitesimal automorphisms of $\zeta$ is surjective with two-dimensional kernel, the canonical map from the space of first-order deformations of $\zeta^{\prime}$ to the space of first-order deformations of $\zeta$ is surjective with two-dimensional kernel, and the obstruction space of $\zeta^{\prime}$ equals the obstruction space of $\zeta$.

Proof. The only claim that does not follow from the long exact sequence of cohomology is that $\mathbb{H}^{0}\left(\Sigma^{\prime}, L_{\left(\zeta^{\prime}, i^{\prime}\right)}^{\vee}\right) \rightarrow \mathbb{H}^{0}\left(\Sigma, L_{(\zeta, i)}^{\vee}\right)$ is surjective. Since the irreducible component $\Sigma_{j} \subset \Sigma$ containing $s$ is stable, Lemma 3.5 states that every infinitesimal automorphism of $\zeta$ vanishes on $\Sigma_{j}$. The infinitesimal automorphisms of $\zeta$ that vanish at $s$ are the same as the infinitesimal automorphisms of $\zeta^{\prime}$ that vanish on $E$. Therefore every infinitesimal automorphism of $\zeta$ is the image of an infinitesimal automorphism of $\zeta^{\prime}$ that vanishes on $E$.

Let $v$ be a morphism of Type Ib. Let $\Sigma_{j} \subset \Sigma$ denote the unstable component containing $s$. Let $N$ denote the one-dimensional vector space $\Theta_{\Sigma_{j}} \otimes_{\mathcal{O}_{\Sigma_{j}}} \mathcal{O}_{s}$.

Lemma 3.17. If $v$ is Type Ib then there are exact sequences,

$$
\begin{array}{r}
0 \rightarrow H^{0}\left(E, T_{E}(-D)\right) \rightarrow \mathbb{H}^{0}\left(\Sigma^{\prime}, L_{\left(\zeta^{\prime}, i^{\prime}\right)}^{\vee}\right) \rightarrow \mathbb{H}^{0}\left(\Sigma, L_{(\zeta, i)}^{\vee}\right) \rightarrow N \rightarrow 0, \\
0 \rightarrow H^{0}\left(E, T_{E} \otimes_{\mathcal{O}_{E}} N_{E / S^{\prime}}\right) / N \rightarrow \mathbb{H}^{1}\left(\Sigma^{\prime}, L_{\left(\zeta^{\prime}, i^{\prime}\right)}^{\vee}\right) \rightarrow \mathbb{H}^{1}\left(\Sigma, L_{(\zeta, i)}^{\vee}\right) \\
0 \rightarrow 0, \\
\rightarrow \mathbb{H}^{2}\left(\Sigma^{\prime}, L_{\left(\zeta^{\prime}, i^{\prime}\right)}^{\vee}\right) \rightarrow \mathbb{H}^{2}\left(\Sigma, L_{(\zeta, i)}^{\vee}\right) \rightarrow 0 .
\end{array}
$$

In other words, the canonical map from the space of infinitesimal automorphisms of $\zeta^{\prime}$ to the space of infinitesimal automorphisms of $\zeta$ has a two-dimensional kernel and a one-dimensional cokernel, the canonical map from the space of first-order deformations of $\zeta^{\prime}$ to the space of first-order deformations 


\section{RATiONAL CURVES ON HYPERSURFACES, II}

of $\zeta$ is surjective with one-dimensional kernel, and the obstruction space of $\zeta^{\prime}$ equals the obstruction space of $\zeta$.

Proof. As in Lemma 3.16, the only claim that does not follow from the long exact sequence of cohomology is that the cokernel of $d u: \mathbb{H}^{0}\left(\Sigma^{\prime}, L_{\left(\zeta^{\prime}, i^{\prime}\right)}^{\vee}\right) \rightarrow \mathbb{H}^{0}\left(\Sigma, L_{(\zeta, i)}^{\vee}\right)$ is $N$. Let $\Sigma_{j}^{\prime} \subset \Sigma^{\prime}$ denote the strict transform of $\Sigma_{j} ; \Sigma_{j}^{\prime}$ is canonically isomorphic to $\Sigma_{j}$. Composing $d u$ with the restriction map $\mathbb{H}^{0}\left(\Sigma, L_{(\zeta, i)}^{\vee}\right) \rightarrow H^{0}\left(\Sigma_{j}, \Theta_{\Sigma_{j}}\right)$ gives a map which is canonically isomorphic to the restriction map $\mathbb{H}^{0}\left(\Sigma^{\prime}, L_{\left(\zeta^{\prime}, i^{\prime}\right)}^{\vee}\right) \rightarrow H^{0}\left(\Sigma_{j}^{\prime}, \Theta_{\Sigma_{j}^{\prime}}\right)$. By Lemma 3.5, every infinitesimal automorphism of $\zeta^{\prime}$ restricts to an infinitesimal automorphism of $\Sigma_{j}^{\prime}$ that vanishes at $s$. On the other hand, the infinitesimal automorphisms of $\zeta$ that vanish at $s$ are the same as the infinitesimal automorphisms of $\zeta^{\prime}$ that vanish on $E$. Hence the image of $d u$ is precisely the subspace of infinitesimal automorphisms of $\zeta$ that vanish at $s$.

Since $\Sigma_{j}$ is unstable, either $\Sigma=\Sigma_{j}$ is a curve of arithmetic genus 1 with no markings and $g$ is constant, or $\Sigma_{j}$ is a smooth curve of genus 0 containing at most two marked points and nodes of $\Sigma$ and $\left.g\right|_{\Sigma_{j}}$ is constant. In each case, it is easy to see that there is an infinitesimal automorphism of $\Sigma_{j}$ that vanishes at all marked points and nodes of $\Sigma$ and that does not vanish at $s$. By Lemma 3.5, this infinitesimal automorphism of $\Sigma_{j}$ is the image of an infinitesimal automorphism of $\zeta$, i.e. there exists an infinitesimal automorphism of $\zeta$ that does not vanish at $s$. Therefore the cokernel of $d u$ is $N$.

Assume next that $v$ is Type II. Because the divisor $D \subset E$ has degree 2, the $\mathcal{O}_{E}$-module $N_{E / S^{\prime}}^{\vee}(-D)$ is isomorphic to $\mathcal{O}_{E}(-1)$. Since $h^{0}\left(E, \mathcal{O}_{E}(-1)\right)=h^{1}\left(E, \mathcal{O}_{E}(-1)\right)=0$, the term $M \otimes_{\mathbb{C}}$ $N_{E / S^{\prime}}^{\vee}(-D)[-1]$ in $K_{\text {II }}$ does not contribute to the hypercohomology; i.e. the hypercohomology of $K_{\mathrm{II}}$ is the sheaf cohomology of $T_{E}(-D)$. The $\mathcal{O}_{E}$-module $T_{E}(-D)$ is isomorphic to $\mathcal{O}_{E}$; hence $h^{0}\left(E, T_{E}(-D)\right)=1$ and $h^{1}\left(E, T_{E}(-D)\right)=0$. Therefore $\operatorname{dim} \mathbb{H}^{0}\left(\Sigma^{\prime}, K_{\mathrm{II}}\right)=H^{0}\left(E, T_{E}(-D)\right)$ is onedimensional, and $\operatorname{dim} \mathbb{H}^{i}\left(\Sigma^{\prime}, K_{\mathrm{II}}\right)=0$ for $k \neq 1$.

For $Q_{\mathrm{II}}$ both $T_{E} \otimes_{\mathcal{O}_{E}} N_{E / S^{\prime}}[0]$ and $M \otimes_{\mathbb{C}} \mathcal{O}_{E}[-1]$ contribute to the hypercohomology. The $\mathcal{O}_{E}$-module $T_{E} \otimes \mathcal{O}_{E} N_{E / S^{\prime}}$ is isomorphic to $\mathcal{O}_{E}(1)$. Hence $\mathbb{H}^{0}\left(\Sigma^{\prime}, Q_{\mathrm{II}}\right)=H^{0}\left(E, T_{E}(E)\right)$ is twodimensional, $\mathbb{H}^{1}\left(\Sigma^{\prime}, Q_{\mathrm{II}}\right)=M$ is one-dimensional (recall $\left.M=\left.\mathcal{O}_{S}(\Sigma)\right|_{s}\right)$, and $\operatorname{dim} \mathbb{H}^{k}\left(\Sigma^{\prime}, Q_{\mathrm{II}}\right)=0$ for $k \neq 0,1$. Therefore there is a long exact sequence in hypercohomology:

$$
\begin{aligned}
& 0 \rightarrow H^{0}\left(E, T_{E}(-D)\right) \rightarrow \mathbb{H}^{0}\left(\Sigma^{\prime}, L_{\left(\zeta^{\prime}, i^{\prime}\right)}^{\vee}\right) \rightarrow \mathbb{H}^{0}\left(\Sigma, L_{(\zeta, i)}^{\vee}\right) \rightarrow \cdots \\
& \cdots \rightarrow H^{0}\left(E, T_{E} \otimes_{\mathcal{O}_{E}} N_{E / S^{\prime}}\right) \rightarrow \mathbb{H}^{1}\left(\Sigma^{\prime}, L_{\left(\zeta^{\prime}, i^{\prime}\right)}^{\vee}\right) \rightarrow \mathbb{H}^{1}\left(\Sigma, L_{(\zeta, i)}^{\vee}\right) \rightarrow \cdots \\
& \cdots \rightarrow M \rightarrow \mathbb{H}^{2}\left(\Sigma^{\prime}, L_{\left(\zeta^{\prime}, i^{\prime}\right)}^{\vee}\right) \rightarrow \mathbb{H}^{2}\left(\Sigma, L_{(\zeta, i)}^{\vee}\right) \rightarrow 0 .
\end{aligned}
$$

Every infinitesimal automorphism of $\zeta$ vanishes at $s$. So the infinitesimal automorphisms of $\zeta$ are the same as the infinitesimal automorphisms of $\zeta^{\prime}$ that vanish on $E$. In particular, $\mathbb{H}^{0}\left(\Sigma^{\prime}, L_{\left(\zeta^{\prime}, i^{\prime}\right)}^{\vee}\right) \rightarrow$ $\mathbb{H}^{0}\left(\Sigma, L_{(\zeta, i)}^{\vee}\right)$ is surjective. The map $\left.\mathbb{H}^{1}\left(\Sigma, L_{(\zeta, i)}^{\vee}\right) \rightarrow \mathcal{O}_{S}(\Sigma)\right|_{s}$ is nonzero if and only if there are deformations of $\zeta$ that smooth the node $s$ to first order. This proves the following two lemmas, which are stated separately for notational convenience.

Lemma 3.18. If $v$ is Type IIa then there are exact sequences,

$$
\begin{array}{r}
0 \rightarrow H^{0}\left(E, T_{E}(-D)\right) \rightarrow \mathbb{H}^{0}\left(\Sigma^{\prime}, L_{\left(\zeta^{\prime}, i^{\prime}\right)}^{\vee}\right) \rightarrow \mathbb{H}^{0}\left(\Sigma, L_{(\zeta, i)}^{\vee}\right) \rightarrow 0, \\
\left.0 \rightarrow H^{0}\left(E, T_{E} \otimes_{\mathcal{O}_{E}} N_{E / S^{\prime}}\right) \rightarrow \mathbb{H}^{1}\left(\Sigma^{\prime}, L_{\left(\zeta^{\prime}, i^{\prime}\right)}^{\vee}\right) \rightarrow \mathbb{H}^{1}\left(\Sigma, L_{(\zeta, i)}^{\vee}\right) \rightarrow \mathcal{O}_{S}(\Sigma)\right|_{s} \\
0 \rightarrow 0, \\
0 \rightarrow \mathbb{H}^{2}\left(\Sigma^{\prime}, L_{\left(\zeta^{\prime}, i^{\prime}\right)}^{\vee}\right) \rightarrow \mathbb{H}^{2}\left(\Sigma, L_{(\zeta, i)}^{\vee}\right) \rightarrow 0 .
\end{array}
$$

In other words, the canonical map from the space of infinitesimal automorphisms of $\zeta^{\prime}$ to the space of infinitesimal automorphisms of $\zeta$ is surjective with a one-dimensional kernel, the canonical map from the space of first-order deformations of $\zeta^{\prime}$ to the space of first-order deformations of $\zeta$ has a 


\section{J. HARRIS AND J. STARR}

two-dimensional kernel and a one-dimensional cokernel, and the obstruction space to $\zeta^{\prime}$ equals the obstruction space to $\zeta$.

Lemma 3.19. If $v$ is Type IIb then there are exact sequences,

$$
\begin{aligned}
& 0 \rightarrow H^{0}\left(E, T_{E}(-D)\right) \rightarrow \mathbb{H}^{0}\left(\Sigma^{\prime}, L_{\left(\zeta^{\prime}, i^{\prime}\right)}^{\vee}\right) \rightarrow \mathbb{H}^{0}\left(\Sigma, L_{(\zeta, i)}^{\vee}\right) \rightarrow 0, \\
& 0 \rightarrow H^{0}\left(E, T_{E} \otimes_{\mathcal{O}_{E}} N_{E / S^{\prime}}\right) \rightarrow \mathbb{H}^{1}\left(\Sigma^{\prime}, L_{\left(\zeta^{\prime}, i^{\prime}\right)}^{\vee}\right) \rightarrow \mathbb{H}^{1}\left(\Sigma, L_{(\zeta, i)}^{\vee}\right) \rightarrow 0, \\
& \left.0 \rightarrow \mathcal{O}_{S}(\Sigma)\right|_{s} \rightarrow \mathbb{H}^{2}\left(\Sigma^{\prime}, L_{\left(\zeta^{\prime}, i^{\prime}\right)}^{\vee}\right) \rightarrow \mathbb{H}^{2}\left(\Sigma, L_{(\zeta, i)}^{\vee}\right) \rightarrow 0 .
\end{aligned}
$$

In other words, the canonical map from the space of infinitesimal automorphisms of $\zeta^{\prime}$ to the space of infinitesimal automorphisms of $\zeta$ is surjective with a one-dimensional kernel, the canonical map from the space of first-order deformations of $\zeta^{\prime}$ to the space of first-order deformations of $\zeta$ is surjective with one-dimensional kernel, and the map from the obstruction space of $\zeta^{\prime}$ to the obstruction space of $\zeta$ is surjective and has a one-dimensional kernel.

Finally, assume that $u: \Sigma^{\prime} \rightarrow \Sigma$ is a Type III elementary morphism, i.e. $u$ is the identity map, but there is one marked point $\sigma^{\prime} \in \Sigma^{\prime}$ that is not in $\Sigma$.

Definition 3.20. Let $u: \Sigma^{\prime} \rightarrow \Sigma$ be a Type III elementary morphism.

i) The morphism $u$ is Type IIIa if $\sigma^{\prime} \in \Sigma$ lies on an unstable component.

ii) The morphism $u$ is Type IIIb if $\sigma^{\prime} \in \Sigma$ lies on a stable component.

In both cases, there is a canonical short exact sequence of complexes:

$$
0 \longrightarrow L_{\zeta} \longrightarrow L_{\zeta^{\prime}} \longrightarrow \Omega_{\Sigma}\left(\sigma^{\prime}\right) \otimes_{\mathcal{O}_{\Sigma}} \mathcal{O}_{\sigma^{\prime}}[0] \longrightarrow 0
$$

Of course $\operatorname{Ext}_{\mathcal{O}_{\Sigma}}^{1}\left(\Omega_{\Sigma}\left(\sigma^{\prime}\right) \otimes_{\mathcal{O}_{\Sigma}} \mathcal{O}_{\sigma^{\prime}}, \mathcal{O}_{\Sigma}\right)$ is canonically isomorphic to $T_{\Sigma} \otimes_{\mathcal{O}_{\Sigma}} \mathcal{O}_{\sigma^{\prime}}$, and $\operatorname{dim} \mathbb{E x t}_{\mathcal{O}_{\Sigma}}^{k}\left(\Omega_{\Sigma}\left(\sigma^{\prime}\right) \otimes_{\mathcal{O}_{\Sigma}} \mathcal{O}_{\sigma^{\prime}}, \mathcal{O}_{\Sigma}\right)=0$ for $k \neq 1$. In particular, there is an induced map

$$
\mathbb{E x t}_{\mathcal{O}_{\Sigma}}^{0}\left(L_{\zeta}, \mathcal{O}_{\Sigma}\right) \longrightarrow T_{\Sigma} \otimes_{\mathcal{O}_{\Sigma}} \mathcal{O}_{\sigma^{\prime}}
$$

This map is zero if and only if $\sigma^{\prime}$ lies on a stable component of $\zeta$. Combined with the long exact sequence of hypercohomology associated to the short exact sequences, this proves the following two lemmas, which are stated separately for notational convenience.

Lemma 3.21. If $u: \Sigma^{\prime} \rightarrow \Sigma$ is Type IIIa then there are exact sequences,

$$
\begin{aligned}
0 \rightarrow \mathbb{E x t}_{\mathcal{O}_{\Sigma^{\prime}}}^{0}\left(L_{\zeta^{\prime}}, \mathcal{O}_{\Sigma^{\prime}}\right) \rightarrow \mathbb{E x t}_{\mathcal{O}_{\Sigma}}^{0}\left(L_{\zeta}, \mathcal{O}_{\Sigma}\right) \rightarrow T_{\Sigma} \otimes_{\mathcal{O}_{\Sigma}} \mathcal{O}_{\sigma^{\prime}} \rightarrow 0, \\
0 \rightarrow \mathbb{E x t}_{\mathcal{O}_{\Sigma^{\prime}}}^{1}\left(L_{\zeta^{\prime}}, \mathcal{O}_{\Sigma^{\prime}}\right) \rightarrow \mathbb{E x t}_{\mathcal{O}_{\Sigma}}^{1}\left(L_{\zeta}, \mathcal{O}_{\Sigma}\right) \rightarrow 0, \\
0 \rightarrow \mathbb{E x t}_{\mathcal{O}_{\Sigma^{\prime}}}^{2}\left(L_{\zeta^{\prime}}, \mathcal{O}_{\Sigma^{\prime}}\right) \rightarrow \mathbb{E x t}_{\mathcal{O}_{\Sigma}}^{2}\left(L_{\zeta}, \mathcal{O}_{\Sigma}\right) \rightarrow 0 .
\end{aligned}
$$

In other words, the space of infinitesimal automorphisms of $\zeta^{\prime}$ maps isomorphically to a codimension-1 linear subspace of the space of infinitesimal automorphisms of $\zeta$, the space of first-order deformations of $\zeta^{\prime}$ equals the space of first-order deformations of $\zeta$, and the obstruction space of $\zeta^{\prime}$ equals the obstruction space of $\zeta$.

Lemma 3.22. If $u: \Sigma^{\prime} \rightarrow \Sigma$ is Type IIIb then there are exact sequences,

$$
\begin{aligned}
0 & \rightarrow \mathbb{E x t}_{\mathcal{O}_{\Sigma^{\prime}}}^{0}\left(L_{\zeta^{\prime}}, \mathcal{O}_{\Sigma^{\prime}}\right) \rightarrow \mathbb{E x t}_{\mathcal{O}_{\Sigma}}^{0}\left(L_{\zeta}, \mathcal{O}_{\Sigma}\right) \rightarrow 0, \\
0 \rightarrow T_{\Sigma} \otimes_{\mathcal{O}_{\Sigma}} \mathcal{O}_{\sigma^{\prime}} & \rightarrow \mathbb{E x t}_{\mathcal{O}_{\Sigma^{\prime}}}^{1}\left(L_{\zeta^{\prime}}, \mathcal{O}_{\Sigma^{\prime}}\right) \rightarrow \mathbb{E x t}_{\mathcal{O}_{\Sigma}}^{1}\left(L_{\zeta}, \mathcal{O}_{\Sigma}\right) \rightarrow 0, \\
0 & \rightarrow \mathbb{E x t}_{\mathcal{O}_{\Sigma^{\prime}}}^{2}\left(L_{\zeta^{\prime}}, \mathcal{O}_{\Sigma^{\prime}}\right) \rightarrow \mathbb{E x t}_{\mathcal{O}_{\Sigma}}^{2}\left(L_{\zeta}, \mathcal{O}_{\Sigma}\right) \rightarrow 0 .
\end{aligned}
$$

In other words, the space of infinitesimal automorphisms of $\zeta^{\prime}$ equals the space of infinitesimal automorphisms of $\zeta$, the canonical map from the space of first-order deformations of $\zeta^{\prime}$ to the space 


\section{RATIONAL CURVES ON HYPERSURFACES, II}

of first-order deformations of $\zeta$ is surjective with one-dimensional kernel, and the obstruction space of $\zeta^{\prime}$ equals the obstruction space of $\zeta^{\prime}$.

Taken together, Lemmas 3.16-3.19, 3.21 and 3.22 describe the canonical maps $\mathbb{E x t}_{\mathcal{O}_{\Sigma^{\prime}}}^{k}\left(L_{\zeta^{\prime}}, \mathcal{O}_{\Sigma^{\prime}}\right) \rightarrow \mathbb{E x t}_{\mathcal{O}_{\Sigma}}^{k}\left(L_{\zeta}, \mathcal{O}_{\Sigma}\right)$ for any morphism $u: \Sigma^{\prime} \rightarrow \Sigma$ that removes a subset of marked points from $\Sigma^{\prime}$ and then contracts a subset of the unstable components.

\subsection{Gluing stable maps}

Let $\tau$ be a stable $A$-graph, not necessarily of genus 0 . To describe the analog of Lemma 3.3, a more precise description of the stack $\overline{\mathcal{M}}(X, \tau)$ is needed. Let $B$ be a scheme. A 1-morphism $\widetilde{\zeta}: B \rightarrow$ $\overline{\mathcal{M}}(X, \tau)$ is equivalent to a pair $(\zeta, \phi)$ consisting of a family of $r$-pointed stable maps,

$$
\zeta=\left(\left(p: \Sigma \rightarrow B, \sigma_{1}, \ldots, \sigma_{r}\right), g: \Sigma \rightarrow X\right),
$$

along with a natural assignment to each geometric point $b \in B$ of a contraction of stable $A$-graphs $\phi_{b}: \tau\left(\zeta_{b}\right) \rightarrow \tau$. The graph $\tau\left(\zeta_{b}\right)$ is the stable $A$-graph of $\left(\left(\Sigma_{b}, \sigma_{1}(b), \ldots, \sigma_{r}(b)\right), g_{b}\right)$. A contraction of stable $A$-graphs, $\phi: \tau^{\prime} \rightarrow \tau$ is a map that contracts subgraphs of $\tau^{\prime}$ to vertices of $\tau$; cf. [BM96, Definition 1.8]. Geometrically the vertices of $\tau$ give a decomposition of $\Sigma_{b}$ into connected subcurves. The main example of a contraction of stable $A$-graphs comes from a family of stable maps over a discrete valuation ring, $\zeta: \operatorname{Spec} R \rightarrow \overline{\mathcal{M}}_{g, r}(X, e)$. Let $\zeta_{0}$ denote the fiber over the geometric closed point of $R$ and let $\zeta_{\eta}$ denote the fiber over the geometric generic point of $R$. There is a canonical contraction $\phi_{(\zeta, R)}: \tau\left(\zeta_{0}\right) \rightarrow \tau\left(\zeta_{\eta}\right)$; a vertex of $\tau\left(\zeta_{0}\right)$ corresponding to an irreducible component $\Sigma_{0, j} \subset \Sigma_{0}$ maps to a vertex of $\tau\left(\zeta_{\eta}\right)$ corresponding to an irreducible component of $\Sigma_{\eta, k} \subset \Sigma_{\eta}$ if and only if $\Sigma_{0, j}$ is in the closure of $\Sigma_{\eta, k}$. The assignment $\phi$ from above is called natural if it is compatible with the action of the Galois group of $\kappa(b)$ and for each map from a discrete valuation ring to $B$, $\rho: \operatorname{Spec} R \rightarrow B$, the contractions $\phi_{\rho(0)}$ and $\phi_{\rho(\eta)}$ commute with the contraction $\phi_{\rho^{*} \zeta, R}$.

Notation 3.23. Associated to each edge $\epsilon=\left\{f_{1}, f_{2}\right\}$ of $\tau$, there is a section $\sigma_{\epsilon}: B \rightarrow \Sigma$ such that for each geometric point $b \in B, \sigma_{\epsilon}(b) \in \Sigma_{b}$ is a node. Denote by $N_{\epsilon}$ the pullback $\sigma_{\epsilon}^{*} \operatorname{Ext}_{\mathcal{O}_{\Sigma}}^{1}\left(\Omega_{p}\left(\sigma_{1}(B)+\cdots+\sigma_{r}(B)\right), \mathcal{O}_{\Sigma}\right)$. Denote by $N_{\tau}$ the direct sum $\bigoplus_{\epsilon} N_{\epsilon}$ where $\epsilon$ ranges over all edges of $\tau$.

Definition 3.24. Let $T$ be a scheme. Let $C$ be a complex of coherent sheaves on $T$ and let $n$ be an integer. The good $(\leqslant r)$-truncation, $C^{\leqslant r}$, is the complex of coherent sheaves on $T$,

$$
\left(C^{\leqslant r}\right)^{k}= \begin{cases}C^{k}, & k<r, \\ \operatorname{Ker}\left(d^{r}: C^{r} \rightarrow C^{r+1}\right), & k=r, \\ \{0\}, & k>r .\end{cases}
$$

The differentials on $C^{\leqslant r}$ are the obvious ones. The association $C \mapsto C \leqslant r$ defines a functor on the category of complexes of coherent sheaves on $T$. This functor takes quasi-isomorphisms to quasiisomorphisms and sends null-homotopic maps to null-homotopic maps; thus it induces a well-defined functor on the derived category of coherent sheaves on $T$. There is a natural transformation to the identity functor, $C^{\leqslant r} \rightarrow C$.

The $\mathcal{O}_{B}$-module $N_{\epsilon}$ is invertible and the localization of $\operatorname{Ext}_{\mathcal{O}_{\Sigma}}^{1}\left(\Omega_{p}\left(\sigma_{1}(B)+\cdots+\sigma_{r}(B)\right), \mathcal{O}_{\Sigma}\right)$ along $\sigma_{\epsilon}(B)$ is canonically isomorphic to $\left(\sigma_{\epsilon}\right)_{*} N_{\epsilon}$. By construction there is a map of complexes of coherent $\mathcal{O}_{\Sigma}$-modules, $L_{\zeta}^{\vee} \rightarrow \mathbb{R H o m}_{\mathcal{O}_{\Sigma}}\left(\Omega_{p}\left(\sigma_{1}(B)+\cdots+\sigma_{r}(B)\right), \mathcal{O}_{\Sigma}\right)$. For all $k>1$, $\operatorname{Ext}_{\mathcal{O}_{B}}^{k}\left(\Omega_{B}\left(\sigma_{1}(B)+\cdots+\sigma_{r}(B)\right), \mathcal{O}_{B}\right)=\{0\}$. So the complex is quasi-isomorphic to its ( $\left.\leqslant 1\right)$-good truncation,

$$
\mathbb{R}^{\leqslant 1} \operatorname{Hom}_{\mathcal{O}_{\Sigma}}\left(\Omega_{p}\left(\sigma_{1}(B)+\cdots+\sigma_{r}(B)\right), \mathcal{O}_{\Sigma}\right) \stackrel{\text { qism }}{\longrightarrow} \mathbb{R H o m}_{\mathcal{O}_{\Sigma}}\left(\Omega_{p}\left(\sigma_{1}(B)+\cdots+\sigma_{r}(B)\right), \mathcal{O}_{\Sigma}\right) .
$$




\section{J. HARRIS AND J. STARR}

Notation 3.25. After replacing $L_{\zeta}^{\vee}$ by a quasi-isomorphic complex, there is a morphism of complexes denoted $a_{(\zeta, \tau)}: L_{\zeta}^{\vee} \rightarrow \mathbb{R}^{\leqslant 1} \operatorname{Hom}_{\mathcal{O}_{\Sigma}}\left(\Omega_{p}\left(\sigma_{1}(B)+\cdots+\sigma_{r}(B)\right), \mathcal{O}_{\Sigma}\right)$ factoring the original morphism. There is a map of complexes of $\mathcal{O}_{\Sigma}$-modules denoted

$$
b_{(\zeta, \tau)}: \mathbb{R}^{\leqslant 1} \operatorname{Hom}_{\mathcal{O}_{\Sigma}}\left(\Omega_{p}\left(\sigma_{1}(B)+\cdots+\sigma_{r}(B)\right), \mathcal{O}_{\Sigma}\right) \longrightarrow \operatorname{Ext}_{\mathcal{O}_{\Sigma}}^{1}\left(\Omega_{p}\left(\sigma_{1}(B)+\cdots+\sigma_{r}(B)\right), \mathcal{O}_{\Sigma}\right)[-1] .
$$

Denote by

$$
c_{(\zeta, \tau)}: \operatorname{Ext}_{\mathcal{O}_{\Sigma}}^{1}\left(\Omega_{p}\left(\sigma_{1}(B)+\cdots+\sigma_{r}(B)\right), \mathcal{O}_{\Sigma}\right)_{\tau} \longrightarrow \operatorname{Ext}_{\mathcal{O}_{\Sigma}}^{1}\left(\Omega_{p}\left(\sigma_{1}(B)+\cdots+\sigma_{r}(B)\right), \mathcal{O}_{\Sigma}\right)
$$

the kernel of

$$
\operatorname{Ext}_{\mathcal{O}_{\Sigma}}^{1}\left(\Omega_{p}\left(\sigma_{1}(B)+\cdots+\sigma_{r}(B)\right), \mathcal{O}_{\Sigma}\right) \longrightarrow \bigoplus_{\epsilon}\left(\sigma_{\epsilon}\right)_{*} N_{\epsilon}
$$

Denote by

$$
d_{(\zeta, \tau)}: \mathbb{R}^{\leqslant 1} \operatorname{Hom}_{\mathcal{O}_{\Sigma}}\left(\Omega_{p}\left(\sigma_{1}(B)+\cdots+\sigma_{r}(B)\right), \mathcal{O}_{\Sigma}\right)_{\tau} \longrightarrow \mathbb{R}^{\leqslant 1} \operatorname{Hom}_{\mathcal{O}_{\Sigma}}\left(\Omega_{p}\left(\sigma_{1}(B)+\cdots+\sigma_{r}(B)\right), \mathcal{O}_{\Sigma}\right)
$$

the fiber product of $b_{(\zeta, \tau)}$ and $c_{(\zeta, \tau)}$. Denote by

$$
e_{(\zeta, \tau)}: L_{(\zeta, \tau)}^{\vee} \longrightarrow L_{\zeta}^{\vee}
$$

the fiber product of $a_{(\zeta, \tau)}$ and $d_{(\zeta, \tau)}$.

Let $|\tau|$ denote the underlying modular graph of $\tau$, i.e. the graph of $\tau$ along with the genus function on vertices, but without the degree function on vertices; cf. [BM96]. There is a (highly nonseparated) Artin stack of $|\tau|$-prestable curves, $\mathfrak{M}(|\tau|)$, and a 1-morphism $\overline{\mathcal{M}}(X, \tau) \rightarrow \mathfrak{M}(|\tau|)$. The relative obstruction theory for this 1-morphism is described in [Beh97]. From this the absolute obstruction theory of $\overline{\mathcal{M}}(X, \tau)$ readily follows.

Lemma 3.26. Let $X$ be a smooth quasi-projective scheme. Let $\tau$ be a stable A-graph. Let

$$
\zeta=\left(\left(p: \Sigma \rightarrow \overline{\mathcal{M}}(X, \tau), \sigma_{1}, \ldots, \sigma_{r}\right), g: \Sigma \rightarrow X\right)
$$

denote the universal family of stable maps over $\overline{\mathcal{M}}(X, \tau)$. There exists an obstruction theory for $\overline{\mathcal{M}}(X, \tau)$ in the sense of [BF98, Definition 4.4] of the form

$$
\left(\mathbb{R} p_{*}\left(L_{(\zeta, \tau)}^{\vee}\right)[1]\right)^{\vee} \longrightarrow L_{\overline{\mathcal{M}}(X, \tau)} .
$$

This obstruction theory is perfect, and there is a distinguished triangle in the derived category of complexes of coherent $\mathcal{O}_{\overline{\mathcal{M}}(X, \tau)}$-modules,

$$
\mathbb{R} p_{*}\left(L_{(\zeta, \tau)}^{\vee}\right)[1] \longrightarrow \mathbb{R} p_{*}\left(L_{\zeta}^{\vee}\right)[1] \longrightarrow N_{\tau} \longrightarrow \mathbb{R} p_{*}\left(L_{\zeta, \tau}^{\vee}\right)[2]
$$

Remark 3.27. The definition of $L_{(\zeta, \tau)}^{\vee}$ seems very complicated, but in fact it is quite simple. Let $\left(\left(\Sigma, \sigma_{1}, \ldots, \sigma_{r}\right), g\right)$ be a stable $\tau$-map. Let $i: \Sigma \rightarrow S$ be a closed immersion from $\Sigma$ to a smooth surface $S$. Let $N_{i}$ denote the normal sheaf of $i$. The complex $L_{\zeta}^{\vee}$ is quasi-isomorphic to the complex $L_{(\zeta, i)}^{\vee}$. There is a surjective map $N_{(\zeta, i)} \rightarrow N_{i}\left(\sigma_{1}(B)+\cdots+\sigma_{r}(B)\right)$. Define $N_{(\zeta, i, \tau)} \subset N_{(\zeta, i)}$ to be the subsheaf which is the inverse image of $\mathcal{I} \cdot N_{i}\left(\sigma_{1}(B)+\cdots+\sigma_{r}(B)\right) \subset N_{i}\left(\sigma_{1}(B)+\cdots+\sigma_{r}(B)\right)$, where $\mathcal{I}$ is the ideal sheaf of $\cup_{\epsilon} \sigma_{\epsilon}$. Then $e_{(\zeta, \tau)}: L_{(\zeta, \tau)}^{\vee} \rightarrow L_{\zeta}^{\vee}$ is quasi-isomorphic to the following map of complexes.

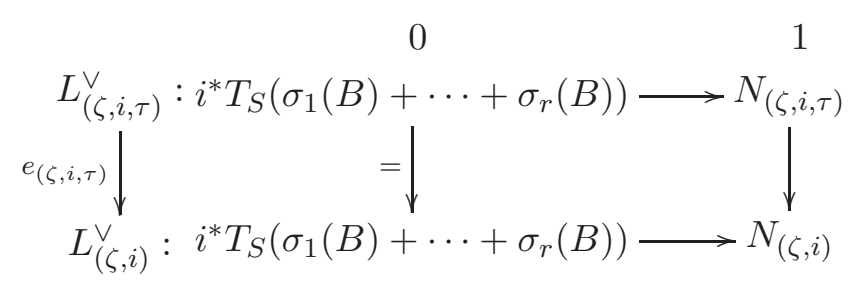




\section{RATIONAL CURVES ON HYPERSURFACES, II}

Let $\epsilon=\left\{f_{1}, f_{2}\right\}$ be a disconnecting edge of $\tau$. Let $\tau_{1} \subset \tau$ be the maximal connected subgraph which contains $f_{1}$ and not $f_{2}$, and let $\tau_{2} \subset \tau$ be the maximal connected subgraph which contains $f_{2}$ and not $f_{1}$. There are 'forgetful' 1-morphisms $F_{i}: \overline{\mathcal{M}}(X, \tau) \rightarrow \overline{\mathcal{M}}\left(X, \tau_{i}\right)$ for $i=1,2$. Let $\widetilde{\zeta}=(\zeta, \phi)$ be a map in $\overline{\mathcal{M}}\left(X, \tau_{i}\right)$, where $\zeta=\left(\left(\Sigma, \sigma_{1}, \ldots, \sigma_{r}\right), g\right)$. Let $\sigma_{\epsilon} \in \Sigma$ be the node corresponding to $\epsilon$. Let $\widetilde{\zeta}_{i}=\left(\zeta_{i}, \phi_{i}\right) \in \overline{\mathcal{M}}\left(X, \tau_{i}\right)$ be the image $F_{i}(\widetilde{\zeta})$. Denote $\zeta_{i}=\left(\left(\Sigma_{i}, \sigma_{i, 0}, \ldots, \sigma_{i, r_{i}}\right), g_{i}\right)$ for $i=1,2$ where $\sigma_{i, 0} \in \Sigma_{i}$ is the point corresponding to the flag $f_{i}$ of $\tau_{i}$, i.e. $\sigma_{i, 0}=\sigma_{\epsilon}$.

\section{LEMMA 3.28. If}

i) the dimension of the obstruction group of $\overline{\mathcal{M}}\left(X, \tau_{i}\right)$ at $\widetilde{\zeta}_{i}$ is $0, i=1,2$, and

ii) the evaluation morphism $\operatorname{ev}_{f_{1}}: \overline{\mathcal{M}}\left(X, \tau_{1}\right) \rightarrow X$ is smooth at $\widetilde{\zeta}_{1}$,

then the dimension of the obstruction group of $\overline{\mathcal{M}}(X, \tau)$ at $\widetilde{\zeta}$ is 0 , and there is a short exact sequence,

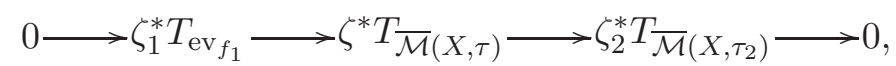

where $T_{\mathrm{ev}_{f_{1}}}$ is the dual of the sheaf of relative differentials of $\mathrm{ev}_{f_{1}}$.

Proof. The proof follows from the fact that $\overline{\mathcal{M}}(X, \tau)$ is an open substack of the 2-fibered product:

$$
\overline{\mathcal{M}}\left(X, \tau_{1}\right) \times_{\mathrm{ev}_{f_{1}}, X \mathrm{ev}_{f_{2}}} \overline{\mathcal{M}}\left(X, \tau_{2}\right) .
$$

Let $\phi: \tau \rightarrow \tau^{\prime}$ be the minimal contraction of stable $A$-graphs that contracts the edge $\left\{f_{1}, f_{2}\right\}$. The induced 1-morphism,

$$
\overline{\mathcal{M}}(X, \phi): \overline{\mathcal{M}}(X, \tau) \longrightarrow \overline{\mathcal{M}}\left(X, \tau^{\prime}\right),
$$

is unramified and the image has codimension at most 1 . In some circumstances, this morphism is the normalization of a Cartier divisor.

\section{LEMMA 3.29. If}

i) $\tau$ has genus 0 ,

ii) the dimension of the obstruction group of $\overline{\mathcal{M}}\left(X, \tau_{2}\right)$ at $\widetilde{\zeta}_{2}$ is 0 , and

iii) the $\mathcal{O}_{\Sigma_{1}}$-module $g_{1}^{*} T_{X}$ is generated by global sections,

then the dimension of the obstruction group of $\overline{\mathcal{M}}\left(X, \tau^{\prime}\right)$ at $\overline{\mathcal{M}}(X, \phi)(\widetilde{\zeta})$ is 0 , the irreducible component of $\overline{\mathcal{M}}(X, \tau)$ containing $\widetilde{\zeta}$ maps to a Cartier divisor under $\overline{\mathcal{M}}(X, \phi)$, and there is a short exact sequence,

$$
\left.\left.\left.\left.0 \longrightarrow T_{\overline{\mathcal{M}}(X, \tau)}\right|_{\tilde{\zeta}} \longrightarrow T_{\overline{\mathcal{M}}\left(X, \tau^{\prime}\right)}\right|_{\widetilde{\zeta}} \longrightarrow \Theta_{\Sigma_{1}}\right|_{\sigma_{1,0}} \otimes \Theta_{\Sigma_{2}}\right|_{\sigma_{2,0}} \longrightarrow 0
$$

Proof. There is a short exact sequence of complexes,

$$
0 \longrightarrow L_{\Sigma}\left(\sigma_{1}+\cdots+\sigma_{r}\right) \longrightarrow L_{\zeta} \longrightarrow g^{*} \Omega_{X}[1] \longrightarrow 0,
$$

that gives rise to a long exact sequence in hypercohomology, part of which is

$$
\left.\mathbb{H}^{1}\left(\Sigma, L_{\zeta}^{\vee}\right) \longrightarrow \mathbb{H}^{1}\left(\Sigma, L_{\Sigma}^{\vee}\left(-\left(\sigma_{1}+\cdots+\sigma_{r}\right)\right)\right) \longrightarrow H^{1}\left(\Sigma, g^{*} T_{X}\right)\right) \longrightarrow \mathbb{H}^{2}\left(\Sigma, L_{\zeta}^{\vee}\right) \longrightarrow 0
$$

The goal is to prove that $\operatorname{dim} \mathbb{H}^{2}\left(\Sigma, L_{\zeta}^{\vee}\right)=0$, i.e. that $\mathbb{H}^{1}\left(\Sigma, L_{\Sigma}^{\vee}\left(-\left(\sigma_{1}+\cdots+\sigma_{r}\right)\right)\right) \rightarrow H^{1}\left(\Sigma, g^{*} T_{X}\right)$ is surjective. After replacing $L_{\Sigma}$ and $L_{\zeta}$ by quasi-isomorphic complexes of locally free $\mathcal{O}_{\Sigma}$-modules, 


\section{J. HARRIS AND J. STARR}

there is a diagram of distinguished triangles of coherent $\mathcal{O}_{B}$-modules

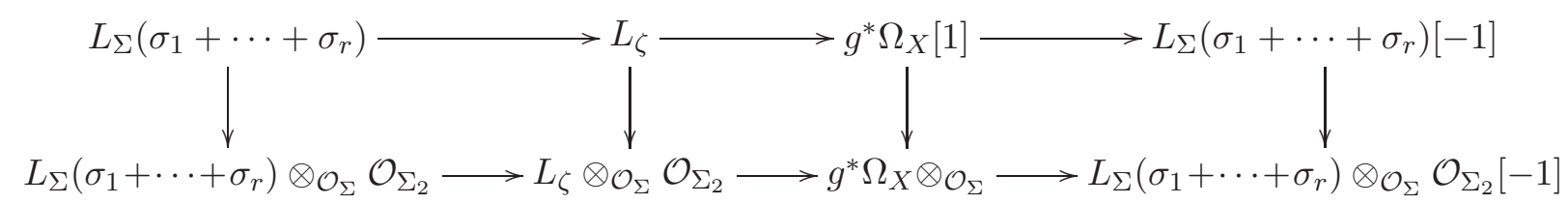

that gives rise to a commutative diagram of exact sequences in hypercohomology:

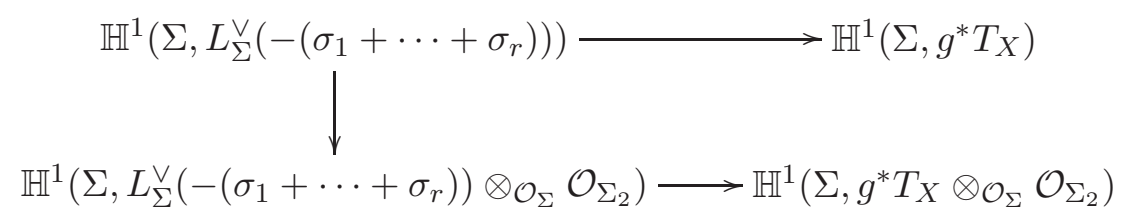

To prove that the top horizontal arrow is surjective, it suffices to prove the following requirements:

i) the left vertical arrow is surjective,

ii) the right vertical arrow is an isomorphism, and

iii) the bottom vertical arrow is surjective.

Let $S$ be a smooth surface, and let $i: \Sigma \rightarrow S$ be an unramified morphism. Then $L_{\Sigma}^{\vee}\left(-\left(\sigma_{1}+\cdots+\sigma_{r}\right)\right)$ is represented in the derived category of coherent sheaves by the following complex:

$$
L_{i}^{\vee}\left(-\left(\sigma_{1}+\cdots+\sigma_{r}\right)\right): i^{*} T_{S}\left(-\left(\sigma_{1}+\cdots+\sigma_{r}\right)\right) \stackrel{\beta_{i}^{\dagger}}{\longrightarrow} N_{i}\left(-\left(\sigma_{1}+\cdots+\sigma_{r}\right)\right)
$$

Let $K$ denote the kernel of $\beta_{i}^{\dagger}$ and let $Q$ denote the cokernel of $\beta_{i}^{\dagger}$. The sheaf $K$ is torsion-free and is locally free of rank 1 on a dense open subset of $\Sigma$. The sheaf $Q$ is torsion. There is a short exact sequence of complexes

$$
0 \longrightarrow K[0] \longrightarrow L_{i}^{\vee}\left(-\left(\sigma_{1}+\cdots+\sigma_{r}\right)\right) \longrightarrow Q[-1] \longrightarrow 0 .
$$

This gives rise to a long exact sequence in hypercohomology, part of which is

$$
H^{1}(\Sigma, K) \longrightarrow \mathbb{H}^{1}\left(\Sigma, L_{i}^{\vee}\left(-\left(\sigma_{1}+\cdots+\sigma_{r}\right)\right)\right) \longrightarrow H^{0}(\Sigma, Q) \longrightarrow 0 .
$$

Let $K^{\prime}$ denote the kernel of $\beta_{i}^{\dagger} \otimes \mathcal{O}_{\Sigma} \mathcal{O}_{\Sigma_{2}}$ and let $Q^{\prime}$ denote the cokernel. There are induced maps of coherent sheaves on $\Sigma, K \rightarrow K^{\prime}$ and $Q \rightarrow Q^{\prime}$. These maps give rise to a commutative diagram with exact rows:

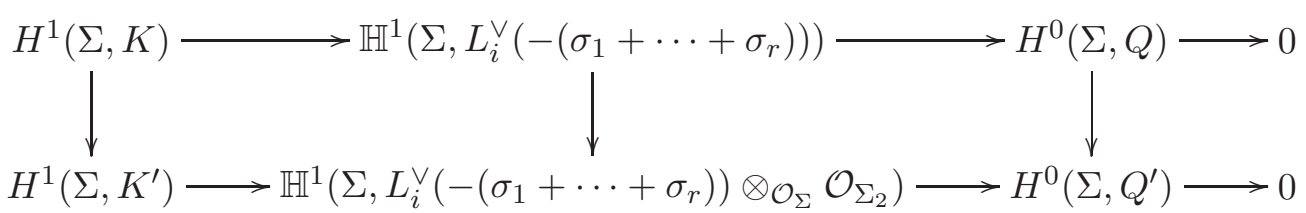

Now $Q \rightarrow Q^{\prime}$ is a surjective map of torsion sheaves, so the map on the right is surjective. The cokernel of $K \rightarrow K^{\prime}$ is torsion, and hence the map on the left is surjective. Therefore the map in the middle is surjective, i.e. requirement $\mathrm{i}$ holds.

There is a short exact sequence of $\mathcal{O}_{\Sigma}$-modules,

$$
0 \longrightarrow\left(g_{1}\right)^{*} T_{X}\left(-\sigma_{1,0}\right) \longrightarrow g^{*} T_{X} \longrightarrow g^{*} T_{X} \otimes_{\mathcal{O}_{\Sigma}} \mathcal{O}_{\Sigma_{2}} \longrightarrow 0 .
$$

By hypothesis $\left(g_{1}\right)^{*} T_{X}$ is generated by global sections; hence $h^{1}\left(\Sigma_{1},\left(g_{1}\right)^{*} T_{X}\left(-\sigma_{1,0}\right)\right)=0$ by Lemma 2.3. Therefore $H^{1}\left(\Sigma, g^{*} T_{X}\right) \rightarrow H^{1}\left(\Sigma, g^{*} T_{X} \otimes_{\mathcal{O}_{\Sigma}} \mathcal{O}_{\Sigma_{2}}\right)$ is an isomorphism, i.e. requirement ii is true. 


\section{RATIONAL CURVES ON HYPERSURFACES, II}

Denote by $j: \Sigma_{2} \rightarrow \Sigma$ the canonical closed immersion. There is a morphism of complexes of $\mathcal{O}_{\Sigma}$-modules:

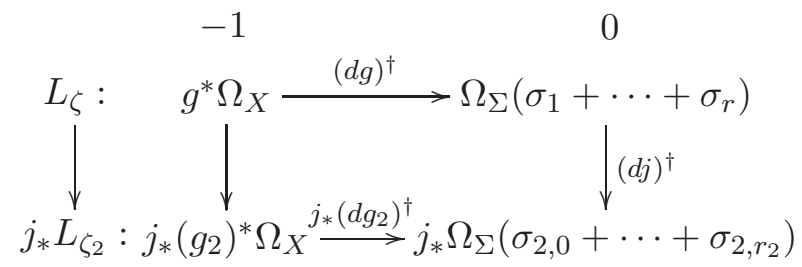

By adjunction, there is a morphism of complexes of $\mathcal{O}_{\Sigma_{2}}$-modules, $L_{\zeta} \otimes_{\mathcal{O}_{\Sigma}} \mathcal{O}_{\Sigma_{2}} \rightarrow L_{\zeta_{2}}$. So there is a commutative diagram in hypercohomology:

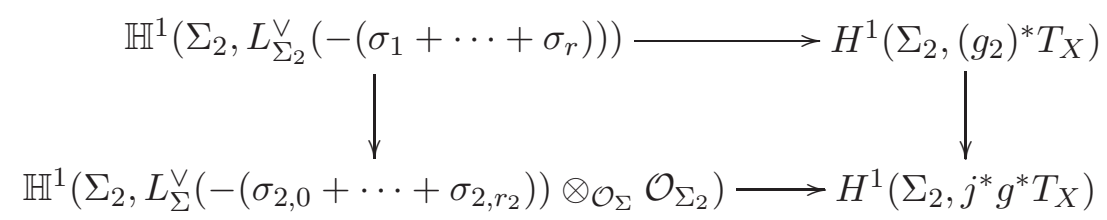

Of course $\left(g_{2}\right)^{*} T_{X}=j^{*} g^{*} T_{X}$, so $H^{1}\left(\Sigma_{2},\left(g_{2}\right)^{*} T_{X}\right) \rightarrow H^{1}\left(\Sigma_{2}, j^{*} g^{*} T_{X}\right)$ is an isomorphism. By hypothesis, the obstruction group of $\zeta_{2}$ vanishes; hence $\mathbb{H}^{1}\left(\Sigma_{2}, L_{\Sigma_{2}}^{\vee}\left(-\left(\sigma_{2,0}+\cdots+\sigma_{2, r_{2}}\right)\right) \rightarrow\right.$ $H^{1}\left(\Sigma_{2},\left(g_{2}\right)^{*} T_{X}\right)$ is surjective. Therefore also $\mathbb{H}^{1}\left(\Sigma_{2}, L_{\Sigma}^{\vee}\left(-\left(\sigma_{1}+\cdots+\sigma_{r}\right)\right) \otimes_{\mathcal{O}_{\Sigma}} \mathcal{O}_{\Sigma_{2}}\right) \rightarrow H^{1}\left(\Sigma_{2}, j^{*} g^{*} T_{X}\right)$ is surjective. This proves requirement iii. Therefore the dimension of the obstruction group of $\overline{\mathcal{M}}\left(X, \tau^{\prime}\right)$ at $\overline{\mathcal{M}}(X, \phi)(\widetilde{\zeta})$ is 0 .

Because the obstruction groups of $\overline{\mathcal{M}}(X, \tau)$ and $\overline{\mathcal{M}}\left(X, \tau^{\prime}\right)$ both vanish, each stack is smooth of the expected dimension at $\widetilde{\zeta}$. The expected dimension of $\overline{\mathcal{M}}(X, \tau)$ is one less than the expected dimension of $\overline{\mathcal{M}}\left(X, \tau^{\prime}\right)$, because $\tau$ has one extra edge (before stabilization). Therefore the image of the irreducible component of $\overline{\mathcal{M}}(X, \tau)$ containing $\widetilde{\zeta}$ is a Cartier divisor in $\overline{\mathcal{M}}\left(X, \tau^{\prime}\right)$.

Finally, the short exact sequence follows from the 2-Cartesian diagram of Artin stacks:

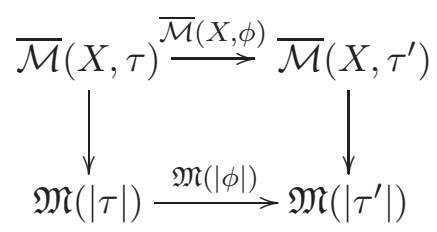

The image of $\mathfrak{M}(|\phi|)$ is a Cartier divisor. Because the image of $\overline{\mathcal{M}}(X, \phi)$ is a Cartier divisor, the normal bundle of $\overline{\mathcal{M}}(X, \phi)$ is simply the pullback of the normal bundle of $\mathfrak{M}(|\phi|)$, which is the bundle whose fiber at $\widetilde{\zeta}$ is the space of first-order deformations of the node $\sigma_{\epsilon} \in \Sigma$. It is well known that this space is canonically isomorphic to $\left.\left.\Theta_{\Sigma_{1}}\right|_{\sigma_{1,0}} \otimes \Theta_{\Sigma_{2}}\right|_{\sigma_{2,0}}$.

Remark 3.30. There is a relative version of Lemma 3.29. Let $B$ be a scheme and let $\widetilde{\zeta}=(\zeta, \phi)$ be a family of $\tau$-maps over $B$. If, for every geometric point $b \in B$, the map $\widetilde{\zeta}_{b}$ satisfies the hypotheses of Lemma 3.29, then the obstruction group of $\overline{\mathcal{M}}(X, \sigma)$ at $\widetilde{\zeta}_{b}$ vanishes, the image of $\overline{\mathcal{M}}(X, \tau)$ in $\overline{\mathcal{M}}\left(X, \tau^{\prime}\right)$ is a Cartier divisor, and there exists a short exact sequence of locally free $\mathcal{O}_{B}$-modules,

$$
0 \longrightarrow \widetilde{\zeta}^{*} T_{\overline{\mathcal{M}}(X, \tau)} \longrightarrow \widetilde{\zeta}^{*} T_{\overline{\mathcal{M}}\left(X, \tau^{\prime}\right)} \longrightarrow \sigma_{1,0}^{*} \Theta_{\Sigma_{1} / B} \otimes_{\mathcal{O}_{B}} \sigma_{2,0}^{*} \Theta_{\Sigma_{2} / B} \longrightarrow 0 .
$$

\section{Conditions on families of stable maps}

This section defines and proves basic results about the many conditions discussed in $\S 1.2$. Because of the process of modification given in the next section, it is necessary to work with families of stable maps that are parametrized by reducible curves of genus 0 . This is the setting in which all definitions are made. 


\section{J. HARRIS AND J. STARR}

Let $\zeta=\left(\left(\Sigma, \sigma_{1}, \ldots, \sigma_{r}\right), g: \Sigma \rightarrow X\right)$ be an $r$-pointed genus-0 stable map to $X$.

Definition 4.1. The stable map $\zeta$ is very stable if the prestable map $(\Sigma, g: B \rightarrow X)$ is stable.

Let $X$ be a quasi-projective scheme. Let $B$ be a scheme. Let $\zeta: B \rightarrow \overline{\mathcal{M}}_{g, r}(X, e)$ be a 1-morphism. Notation 4.2. The 1-morphism $\zeta: B \rightarrow \overline{\mathcal{M}}_{g, r}(X, e)$ consists of a datum,

$$
\zeta=\left(\left(p_{\zeta}: \Sigma_{\zeta} \rightarrow B, \sigma_{(\zeta, 1)}, \ldots, \sigma_{(\zeta, r)}\right), g_{\zeta}: \Sigma \rightarrow X\right),
$$

where

i) $p_{\zeta}: \Sigma_{\zeta} \rightarrow B$ is a proper, flat family of connected prestable curves of arithmetic genus $g$,

ii) $\sigma_{(\zeta, i)}: B \rightarrow \Sigma_{\zeta}, i=1, \ldots, r$, is a collection of everywhere disjoint sections with image in the smooth locus of $p_{\zeta}$, and

iii) $g_{\zeta}: \Sigma_{\zeta} \rightarrow X$ is a morphism of schemes,

that satisfies the stability condition in Definition 3.1. Denote by $h_{\zeta, i}: B \rightarrow X$ the composition $g_{\zeta} \circ \sigma_{(\zeta, i)}$. When there is no risk of confusion, the subscript $\zeta$ will be omitted.

Let $X$ be a smooth, quasi-projective variety. Let $\pi: B \rightarrow T$ be a proper, flat family of connected prestable curves of arithmetic genus 0 . Let $\zeta: B \rightarrow \overline{\mathcal{M}}_{0,1}(X, 1)$ be a 1-morphism $((p: \Sigma \rightarrow B, \sigma), f:$ $\Sigma \rightarrow X)$.

Definition 4.3. The 1-morphism $\zeta: B \rightarrow \overline{\mathcal{M}}_{0,1}(X, 1)$ is twisting (respectively very twisting) if:

i) $(\pi: B \rightarrow T, h: B \rightarrow X)$ is a family of stable maps to $X$, i.e. a 1 -morphism $\xi: T \rightarrow \overline{\mathcal{M}}_{0,0}(X, e)$ for some $e \geqslant 0$,

ii) the dimension of the obstruction group of $\overline{\mathcal{M}}_{0,0}(X, e)$ at each point of $\xi(T)$ is 0 ,

iii) the dimension of the relative obstruction group of the evaluation morphism ev $: \overline{\mathcal{M}}_{0,1}(X, 1) \rightarrow$ $X$ at each point of $\zeta(T)$ is 0 ,

iv) denoting by $T_{\mathrm{ev}}$ the dual of the sheaf of relative differentials $\Omega_{\mathrm{ev}}$, the pullback $\zeta^{*} T_{\mathrm{ev}}$ is $\pi$-relatively generated by global sections (respectively $\pi$-relatively deformation ample), and

v) denoting by pr: $\overline{\mathcal{M}}_{0,1}(X, 1) \rightarrow \overline{\mathcal{M}}_{0,0}(X, 1)$ the projection map, and denoting by $T_{\text {pr }}$ the dual of the sheaf of relative differentials $\Omega_{\mathrm{pr}}$, the pullback $\zeta^{*} T_{\mathrm{pr}}$ is $\pi$-relatively generated by global sections, i.e. the line bundle $\sigma^{*} \mathcal{O}_{\Sigma}(\sigma(B))$ is $\pi$-relatively generated by global sections.

\section{Remark 4.4.}

i) The sheaf $\zeta^{*} T_{\mathrm{pr}}$ is canonically isomorphic to $\sigma^{*} \mathcal{O}_{\Sigma}(\sigma(B))$.

ii) The product morphism $(p, g): \Sigma \rightarrow B \times X$ is a closed immersion whose ideal sheaf is everywhere locally defined by a regular sequence, i.e. it is a regular embedding. Denote by $\mathcal{N}$ the normal bundle of this regular embedding. Then condition iii of Definition 4.3 is equivalent to the condition that $R^{1} p_{*}(\mathcal{N}(-\sigma(B)))=\{0\}$. Under this hypothesis, $\zeta^{*} T_{\text {ev }}$ is canonically isomorphic to $p_{*}(\mathcal{N}(-\sigma(B)))$.

iii) Condition ii of Definition 4.3 is superfluous. Since the prestable family of maps $(\pi: B \rightarrow T, \xi$ : $B \rightarrow X)$ is stable, also $\left(\pi: B \rightarrow T, \zeta: B \rightarrow \overline{\mathcal{M}}_{0,1}(X, 1)\right)$ is stable by Lemma 3.6, part i.

iv) The conditions in Definition 4.3 impose some restrictions on the degrees of the locally free sheaves involved. By [Pan99, Lemma 2.2.2], the total degree of $\sigma^{*} \mathcal{O}_{\Sigma}(\sigma(B))$ is simply $\delta=2 e-e^{\prime}$ where $e$ is the degree of $h: B \rightarrow \mathbb{P}^{N}$ and $e^{\prime}$ is the degree of $f: \Sigma \rightarrow \mathbb{P}^{N}$. If $\zeta$ is twisting then $2 e \geqslant e^{\prime}$.

Let $B$ be a prestable, connected curve of arithmetic genus 0. Let $B_{1}, B_{2} \subset B$ be connected subcurves of $B$ such that $B_{1} \cap B_{2}=\{q\}$ is a single node, and $B=B_{1} \cup B_{2}$. Let $\zeta: B \rightarrow \overline{\mathcal{M}}_{0,1}(X, 1)$ be a 1-morphism. 


\section{RATIONAL CURVES ON HYPERSURFACES, II}

Lemma 4.5. If $\left.\zeta\right|_{B_{i}}: B_{i} \rightarrow \overline{\mathcal{M}}_{0,1}(X, 1)$ is twisting for $i=1,2$, then $\zeta$ is twisting. If both $\zeta_{1}, \zeta_{2}$ are twisting, and if at least one of them is very twisting, then $\zeta$ is very twisting.

Proof. This follows from Lemma 2.11.

Let $\pi: B \rightarrow T$ be a proper, flat family of connected, prestable curves of arithmetic genus 0 . Let $\zeta: B \rightarrow \overline{\mathcal{M}}_{0,1}(X, 1)$ be a 1-morphism. For every morphism of schemes $g: T^{\prime} \rightarrow T$, denote by $\pi_{T^{\prime}}: B_{T^{\prime}} \rightarrow T^{\prime}$ the base change of $\pi$ by $g$, and denote by $\zeta_{T^{\prime}}: B_{T^{\prime}} \rightarrow \overline{\mathcal{M}}_{0,1}(X, 1)$ the composition of $B_{T^{\prime}} \rightarrow B$ with $\zeta$.

Lemma 4.6. There is an open subscheme $U_{\text {twist }} \subset T$ (respectively $U_{\text {vtwist }} \subset T$ ) with the following property: for every morphism of schemes $g: T^{\prime} \rightarrow T$, the pullback family $\pi_{T^{\prime}}: B_{T^{\prime}} \rightarrow T^{\prime}$ and $\zeta_{T^{\prime}}$ : $B_{T^{\prime}} \rightarrow \overline{\mathcal{M}}_{0,1}(X, 1)$ is twisting (respectively very twisting) if and only if $g\left(T^{\prime}\right) \subset U_{\text {twist }}$ (respectively $\left.f\left(T^{\prime}\right) \subset U_{\text {vtwist }}\right)$.

Proof. By [Beh97, Lemma 1] there is an open subscheme $U_{1} \subset T$ with the property that, for every morphism $g,\left(\pi_{T^{\prime}}: B_{T^{\prime}} \rightarrow T^{\prime}, h_{T^{\prime}}: B_{T^{\prime}} \rightarrow X\right)$ is a family of stable maps if and only if $g\left(T^{\prime}\right) \subset U_{1}$. For every morphism $g$ such that $\zeta_{T^{\prime}}$ is twisting, $g\left(T^{\prime}\right) \subset U_{1}$. Hence the lemma for $\zeta_{U_{1}}$ implies the lemma for $\zeta$. After replacing $T$ by $U_{1}$, the morphism $h: B \rightarrow X$ is a family of stable maps over $T$, i.e. a 1-morphism $\xi: T \rightarrow \overline{\mathcal{M}}_{0,0}(X, e)$ for some integer $e$.

Because $\pi$ has relative dimension 1 , the cohomology sheaf $\mathcal{H}^{k}\left(\mathbb{R} \pi_{*}\left(L_{h}^{\vee}\right)\right)=\{0\}$ for $k \geqslant 2$. By cohomology and base change, for every geometric point $t$ of the support of the sheaf $\mathcal{H}^{1}\left(\mathbb{R} \pi_{*}\left(L_{h}^{\vee}\right)\right)$, $\operatorname{dim} \mathbb{H}^{1}\left(B_{t}, L_{h_{t}}^{\vee}\right)>0$, i.e. the obstruction group of $\xi_{t}$ does not vanish. Therefore the dimension of the obstruction group of $\overline{\mathcal{M}}_{0,0}(X, e)$ at each point of $\xi_{T^{\prime}}\left(T^{\prime}\right)$ is 0 if and only if $g\left(T^{\prime}\right)$ is contained in the complement of the support of $\mathcal{H}^{1}\left(\mathbb{R} \pi_{*}\left(L_{h}^{\vee}\right)\right)$. The complement of the support of this sheaf is an open subset of $T$. After replacing $T$ by this open set, the dimension of the obstruction group of $\overline{\mathcal{M}}_{0,0}(X, e)$ at each point of $\xi(T)$ is 0 .

For similar reasons, $T$ can be replaced by the complement of

$$
\pi\left[\operatorname{Supp}\left(R^{1} p_{*}(\mathcal{N}(-\sigma(B)))\right)\right]
$$

where $\mathcal{N}$ is as in part ii of Remark 4.4. After replacing $T$ by this open subset, for every $g, \zeta_{T^{\prime}}$ satisfies conditions i, ii, and iii of Definition 4.3. In order that condition v is satisfied, it is necessary and sufficient that $g\left(T^{\prime}\right)$ is contained in the complement of the support of the sheaf

$$
\operatorname{Coker}\left(\pi^{*} \pi_{*} \sigma^{*} \mathcal{O}_{\Sigma}(\sigma) \rightarrow \sigma^{*} \mathcal{O}_{\Sigma}(\sigma)\right) .
$$

After replacing $T$ by the complement of the support of this sheaf, for every $g, \zeta_{T^{\prime}}$ satisfies conditions i, ii, iii and v of Definition 4.3.

Define $U_{\text {twist }}$ to be the complement in $T$ of the image under $\pi$ of the support of

$$
\operatorname{Coker}\left(\pi^{*} \pi_{*} \zeta^{*} T_{\mathrm{ev}} \rightarrow \zeta^{*} T_{\mathrm{ev}}\right) .
$$

For every morphism $g,\left(\zeta^{*} T_{\mathrm{ev}}\right)_{T^{\prime}}$ is $\pi_{T^{\prime}}$-relatively generated by global sections if and only if $g\left(T^{\prime}\right) \subset$ $U_{\text {twist }}$. Therefore, for every morphism $g, \zeta_{T^{\prime}}$ is twisting if and only if $g\left(T^{\prime}\right) \subset U_{\text {twist }}$. By Lemma 2.9, there exists an open subset $U_{\text {vtwist }} \subset T$ such that for every $g,\left(\zeta^{*} T_{\mathrm{ev}}\right)_{T^{\prime}}$ is $\pi_{T^{\prime}}$-relatively deformation ample if and only if $g\left(T^{\prime}\right) \subset U_{\text {vtwist }}$. Therefore $\zeta_{T^{\prime}}$ is very twisting if and only if $g\left(T^{\prime}\right) \subset U_{\text {vtwist }}$.

Let $(\pi: B \rightarrow T, h: B \rightarrow X)$ be a family of genus-0 stable maps, i.e. a 1-morphism $\xi: T \rightarrow$ $\overline{\mathcal{M}}_{0,0}(X, e)$ for some $e \geqslant 0$. For every morphism of schemes $g: T^{\prime} \rightarrow T$, denote by $\pi_{T^{\prime}}: B_{T^{\prime}} \rightarrow T^{\prime}$ the base change of $\pi$ by $g$, and denote by $\xi_{T^{\prime}}: B_{T^{\prime}} \rightarrow \overline{\mathcal{M}}_{0,1}(X, 1)$ the composition of $B_{T^{\prime}} \rightarrow B$ with $\xi$. 


\section{J. HARRIS AND J. STARR}

Definition 4.7. The family of stable maps $\xi: T \rightarrow \overline{\mathcal{M}}_{0,0}(X, e)$ is twistable (respectively very twistable) if there exists a surjective étale morphism $u: T^{\prime} \rightarrow T$ and a morphism $\zeta: B_{T^{\prime}} \rightarrow$ $\overline{\mathcal{M}}_{0,1}(X, 1)$ such that $\xi_{\zeta}=\xi_{T^{\prime}}$ and such that $\zeta$ is twisting (respectively very twisting).

Proposition 4.8. There is an open subscheme $U_{\text {t-able }} \subset T$ (respectively $U_{\text {vt-able }} \subset T$ ) such that for every morphism of schemes $g: T^{\prime} \rightarrow T, \xi_{T^{\prime}}$ is twistable (respectively very twistable) if and only if $g\left(T^{\prime}\right) \subset U_{\text {t-able }}\left(\right.$ respectively $\left.g\left(T^{\prime}\right) \subset U_{\text {vt-able }}\right)$.

Proof. It suffices to check that, if $t \in T$ is a geometric point such that $h_{t}: B_{t} \rightarrow X$ is twistable (respectively very twistable), then there is an étale neighborhood of $t \in T$ over which $\xi$ is twistable (respectively very twistable). Denote by $\zeta_{t}: B_{t} \rightarrow \overline{\mathcal{M}}_{0,1}(X, 1)$ the twisting morphism. Consider $\overline{\mathcal{M}}_{0,1}(X, 1)$ as a quasi-projective scheme via the Plücker and Segre embeddings of $\mathbb{G}(1, n) \times$ $\mathbb{P}^{n} \hookrightarrow \mathbb{P}^{\frac{1}{2} n(n+1)^{2}-1}$. Let $\beta$ denote the degree of the stable map $\zeta_{t}$.

Define $\mathcal{M}=T \times \overline{\mathcal{M}}_{0,0}\left(\overline{\mathcal{M}}_{0,1}(X, 1), \beta\right)$, i.e. $\mathcal{M}$ parametrizes pairs $(s, \zeta)$ consisting of a point $s \in T$ together with a genus-0 stable map $\zeta: B \rightarrow \overline{\mathcal{M}}_{0,1}(X, 1)$ of degree $\beta$. Denote the universal stable map over $\overline{\mathcal{M}}_{0,0}\left(\overline{\mathcal{M}}_{0,1}(X, 1), \beta\right)$ by

$$
\begin{gathered}
\rho: \mathcal{B} \rightarrow \overline{\mathcal{M}}_{0,0}\left(\overline{\mathcal{M}}_{0,1}(X, 1), \beta\right), \\
\zeta: \mathcal{B} \rightarrow \overline{\mathcal{M}}_{0,1}(X, 1) .
\end{gathered}
$$

As in Notation 4.2 , let $p: \Sigma \rightarrow \mathcal{B}$ be the pullback by $\zeta$ of the universal curve over $\overline{\mathcal{M}}_{0,1}(X, 1)$, let $\sigma: \mathcal{B} \rightarrow \Sigma$ be the pullback of the universal section, let $g: \Sigma \rightarrow X$ be the pullback of the universal map, and let $h=g \circ \sigma$. This gives a family of prestable maps,

$$
\widetilde{\xi}=\left(\rho: \mathcal{B} \rightarrow \overline{\mathcal{M}}_{0,0}\left(\overline{\mathcal{M}}_{0,1}(X, 1), \beta\right), h: \mathcal{B} \rightarrow X\right) .
$$

By [Beh97, Lemma 1] there is a maximal open substack $\mathcal{U}_{e} \subset \overline{\mathcal{M}}_{0,0}\left(\overline{\mathcal{M}}_{0,1}(X, 1), \beta\right)$ over which $\widetilde{\xi}$ is stable of degree $e$. By hypothesis, $\left(t, \zeta_{t}\right)$ is in $T \times \mathcal{U}_{e}$.

Because $\zeta_{t}$ is twisting, the hypotheses of Lemma 3.6, part iii are satisfied where $f: X \rightarrow Y$ corresponds to ev : $\overline{\mathcal{M}}_{0,1}(X, 1) \rightarrow X$ and where $(\Sigma, g: \Sigma \rightarrow X)$ corresponds to $\left(B_{t}, \zeta_{t}: B_{t} \rightarrow\right.$ $\left.\overline{\mathcal{M}}_{0,1}(X, 1)\right)$. Therefore at the point $\left(B_{t}, \zeta_{t}: B_{t} \rightarrow \overline{\mathcal{M}}_{0,1}(X, 1)\right)$, the stack $\mathcal{U}_{e}$ is smooth and the morphism $\widetilde{\xi}: \mathcal{U}_{e} \rightarrow \overline{\mathcal{M}}_{0,0}(X, e)$ is smooth by the Jacobian criterion.

Consider the 1-morphism $\left(1_{T}, \xi\right): T \rightarrow T \times \overline{\mathcal{M}}_{0,0}(X, e)$. Denote by $\mathcal{M}$ the 2 -fibered product of the 1-morphism $\left(1_{T}, \xi\right)$ and the 1-morphism $\left(1_{T}, \widetilde{\xi}\right): T \times \mathcal{U}_{e} \rightarrow T \times \overline{\mathcal{M}}_{0,0}(X, e)$. The 2-fibered product $\mathcal{M}$ is the stack whose objects are triples $(t, \zeta, \theta)$ consisting of a point $t \in T$, an object $\zeta: B \rightarrow$ $\overline{\mathcal{M}}_{0,1}(X, 1)$ of $\mathcal{U}_{e}$, and an equivalence $\theta: \xi_{t} \rightarrow \widetilde{\zeta}$ of objects in the groupoid $\overline{\mathcal{M}}_{0,0}(X, e)(\operatorname{Spec} \kappa(t))$. Because $\left(1_{T}, \widetilde{\xi}\right)$ is smooth at $\left(t,\left(B_{t}, \zeta_{t}\right)\right)$, the projection $\operatorname{pr}_{1}: \mathcal{M} \rightarrow T$ is smooth at $\left(t,\left(B_{t}, \zeta_{t}\right)\right)$. Hence there exists an étale 1-morphism $f: M \rightarrow \mathcal{M}$ from a scheme $M$ to $\mathcal{M}$ such that $\left(t,\left(B_{t}, \zeta_{t}\right)\right)$ is in the image of $f$ and such that the composite morphism $\operatorname{pr}_{1} \circ f: M \rightarrow T$ is smooth. By [BLR90, Proposition 2.2.14], there exists an étale morphism $u:\left(T^{\prime}, t^{\prime}\right) \rightarrow(T, t)$ and a section $z: T^{\prime} \rightarrow M$ such that $f\left(z\left(t^{\prime}\right)\right)$ is $\left(t,\left(B_{t}, \zeta_{t}\right)\right)$. Denote by $\zeta_{\text {pre }}: T^{\prime} \rightarrow \mathcal{U}_{e}$ the composition $\operatorname{pr}_{2} \circ f \circ z$.

Denote by $\zeta: B^{\prime} \rightarrow \overline{\mathcal{M}}_{0,1}(X, 1)$ the pullback by $\zeta_{\text {pre }}: T^{\prime} \rightarrow \mathcal{U}_{e}$ of the universal stable map. By construction, $\widetilde{\xi}(\zeta): B^{\prime} \rightarrow \overline{\mathcal{M}}_{0,0}(X, e)$ is equivalent to $u^{*} \xi: u^{*} B \rightarrow \overline{\mathcal{M}}_{0,0}(X, e)$. Hence, after replacing $T^{\prime}$ by a surjective, étale cover, $B^{\prime}=u^{*} B$ as $T^{\prime}$-schemes, and $\widetilde{\xi}(\zeta)=u^{*} \xi$. The fiber of $\zeta: u^{*} B \rightarrow \overline{\mathcal{M}}_{0,1}(X, 1)$ over every preimage of $\left(t,\left(B_{t}, \zeta_{t}\right)\right)$ is twisting. So by Lemma 4.6 , after replacing $T^{\prime}$ by a Zariski open subscheme whose image contains $t, \zeta: u^{*} B \rightarrow \overline{\mathcal{M}}_{0,1}(X, 1)$ is twisting. Similarly, if $\left(t,\left(B_{t}, \zeta_{t}\right)\right)$ is very twisting, after replacing $T^{\prime}$ by a Zariski open subscheme whose image contains $t, \zeta$ is very twisting. On the Zariski open subscheme of $T$ that is the image of $u: T^{\prime} \rightarrow T$, the family $\xi: B \rightarrow \overline{\mathcal{M}}_{0,0}(X, e)$ is twistable (respectively very twistable). 


\section{RATIONAL CURVES ON HYPERSURFACES, II}

Let

$$
\xi_{i}=\left(\left(\pi_{i}: B_{i} \rightarrow T, \sigma_{i}: T \rightarrow B_{i}\right), h_{i}: B_{i} \rightarrow X\right), \quad i=1,2,
$$

be two families of 1-pointed stable maps such that $h_{1} \circ \sigma_{1}=h_{2} \circ \sigma_{2}$. Denote by

$$
\xi=(\pi: B \rightarrow T, h: B \rightarrow X)
$$

the family obtained by taking $B$ to be the union of $B_{1}$ and $B_{2}$ where the section $\sigma_{1}$ is identified with the section $\sigma_{2}$.

Lemma 4.9. If for $i=1,2$ the family of unpointed stable maps $\left(\pi_{i}: B_{i} \rightarrow T, h_{i}: B_{i} \rightarrow X\right)$ is twistable, and if for every geometric point $t \in T$, the variety parametrizing free lines that contain $h(\sigma(t))$ is irreducible, then $\xi$ is twistable. If also one of $\xi_{1}, \xi_{2}$ is very twistable, then $\xi$ is very twistable.

Proof. This follows from Lemma 4.5. First of all, using Proposition 4.8, it suffices to prove the result when $T=$ Spec $k$ for some algebraically closed field $k$.

For each of $i=1,2$, let $\mathcal{M}_{i}$ denote the 2-fibered product constructed in the proof of Proposition 4.8 , i.e. the objects of $\mathcal{M}_{i}$ are pairs $\left(\zeta_{i}, \theta_{i}\right)$ consisting of a twisting family (respectively very twisting family), $\zeta_{i}: B_{i} \rightarrow \overline{\mathcal{M}}_{0,1}(X, 1)$, such that the induced map

$$
\widetilde{\zeta}_{i}=\left(\left(B_{i}, \sigma_{i}\right), g_{i} \circ \rho_{i}: B_{i} \rightarrow X\right)
$$

is stable, and an equivalence of objects, $\theta_{i}: \xi_{i} \rightarrow \widetilde{\zeta}_{i}$. Since each of $\xi_{i}$ is twistable, each of $\mathcal{M}_{i}$ is nonempty.

By the proof of Proposition 4.8, each of $\mathcal{M}_{i}$ is smooth. By the definition of twisting families, for each $i=1,2$ the morphism

$$
e_{i}: \mathcal{M}_{i} \longrightarrow \overline{\mathcal{M}}_{0,1}(X, 1), \zeta_{i} \longmapsto \zeta_{i}\left(\sigma_{i}\right)
$$

has image contained in the unobstructed locus of ev $: \overline{\mathcal{M}}_{0,1}(X, 1) \rightarrow X$. Let $P \subset \overline{\mathcal{M}}_{0,1}(X, 1)$ denote the preimage under ev of the point $p=h_{1}\left(\sigma_{1}\right)=h_{2}\left(\sigma_{2}\right)$. The image of $e_{i}$ is contained in the smooth locus of $P$. The claim is that $e_{i}: \mathcal{M}_{i} \rightarrow P$ is smooth. The obstruction space at a point $\zeta_{i}$ is a quotient of the cohomology group $H^{1}\left(B_{i}, \zeta_{i}^{*} T_{\mathrm{ev}}\left(-\sigma_{i}\right)\right)$. By Definition 4.3, condition iv, $\zeta_{i}^{*} T_{\mathrm{ev}}$ is generated by global sections. Hence, by Lemma 2.3, part ii, this cohomology group is zero, and $e_{i}$ is smooth.

Since both $e_{1}: \mathcal{M}_{1} \rightarrow P$ and $e_{2}: \mathcal{M}_{2} \rightarrow P$ are smooth, both have nonempty, open image contained in the locus of free lines. By hypothesis, the open subset of $P$ parametrizing free lines is irreducible. Therefore the image of $e_{1}$ and the image of $e_{2}$ intersect. Choose a family $\zeta_{1} \in \mathcal{M}_{1}$ and $\zeta_{2} \in \mathcal{M}_{2}$ such that $e_{1}\left(\zeta_{1}\right)=e_{2}\left(\zeta_{2}\right)$. Then $\zeta_{1}$ and $\zeta_{2}$ can be glued to obtain a morphism $\zeta: B \rightarrow \overline{\mathcal{M}}_{0,1}(X, 1)$ such that $\left.\zeta\right|_{B_{1}}=\zeta_{1}$ and $\left.\zeta\right|_{B_{2}}=\zeta_{2}$. By Lemma 4.5, $\zeta$ is twisting. Moreover, if one of $\zeta_{1}, \zeta_{2}$ is very twisting, then $\zeta$ is very twisting, and $\widetilde{\zeta}=\xi$. Therefore $\xi$ is twistable, and it is very twistable if one of $\xi_{1}, \xi_{2}$ is very twistable.

Hypothesis 4.10. Let $U \subset \overline{\mathcal{M}}_{0,1}(X, 1)$ denote the preimage of $U_{\text {t-able }} \subset \overline{\mathcal{M}}_{0,0}(X, 1)$ under pr. The evaluation morphism ev : $U \rightarrow X$ has Zariski dense image, i.e. a general point of $X$ is contained in a twistable line.

Let $\pi: B \rightarrow T$ be a family of prestable curves of arithmetic genus 0 .

Definition 4.11. A 1-morphism $\zeta: B \rightarrow \overline{\mathcal{M}}_{0,1}(X, e)$ is positive (respectively very positive) if:

i) $(\pi: B \rightarrow T, h: B \rightarrow X)$ is a family of stable maps, i.e. a 1-morphism $\xi: T \rightarrow \overline{\mathcal{M}}_{0,0}(X, \epsilon)$ for some $\epsilon \geqslant 0$, 


\section{J. HARRIS AND J. STARR}

ii) the dimension of the obstruction group of $\overline{\mathcal{M}}_{0,0}(X, \epsilon)$ is 0 at each point of $\xi(T)$,

iii) the dimension of the obstruction group of $\overline{\mathcal{M}}_{0,0}(X, e)$ is 0 at each point of $\operatorname{pr}(\zeta(T))$,

iv) the pullback $(\mathrm{pr} \circ \zeta)^{*} T_{\overline{\mathcal{M}}_{0,0}(X, e)}$ is $\pi$-relatively deformation ample, and

v) the pullback $\sigma^{*} \mathcal{O}_{\Sigma}(\sigma)$ is $\pi$-relatively generated by global sections (respectively $\pi$-relatively ample).

Remark 4.12. This definition is similar to Definition 4.3. It differs in that e need not equal 1 and that pro $\zeta$ has image in the unobstructed locus, instead of requiring $\zeta$ to have image in the unobstructed locus of the morphism ev.

Lemma 4.13. Let $\pi: B \rightarrow T$ be a family of prestable curves of arithmetic genus 0 and let $\zeta$ : $B \rightarrow \overline{\mathcal{M}}_{0,1}(X, 1)$ be a 1-morphism. There is an open subscheme $U_{\text {pos }} \subset T$ (respectively $U_{\text {vpos }} \subset T$ ) with the following property: for every morphism of schemes $f: T^{\prime} \rightarrow T$, the pullback family $f^{*} \pi: f^{*} B \rightarrow T^{\prime}$ and $f^{*} \zeta: f^{*} B \rightarrow \overline{\mathcal{M}}_{0,1}(X, 1)$ is positive (respectively very positive) if and only if $f\left(T^{\prime}\right) \subset U_{\text {pos }}$ (respectively $\left.f\left(T^{\prime}\right) \subset U_{\text {vpos }}\right)$.

Proof. The proof is almost identical to the proof of Lemma 4.6.

Lemma 4.14. Let $B$ be a prestable curve of arithmetic genus 0 , and let $\zeta: B \rightarrow \overline{\mathcal{M}}_{0,1}(X, e)$ be a positive 1-morphism whose image is contained in the locus of very stable maps.

i) If $B$ is smooth, then $\zeta: B \rightarrow \overline{\mathcal{M}}_{0,1}(X, e)$ is free, i.e. $\zeta^{*} T_{\overline{\mathcal{M}}_{0,1}(X, e)}$ is generated by global sections. If $\zeta$ is very positive, then $\zeta$ is very free, i.e. $\zeta^{*} T_{\overline{\mathcal{M}}_{0,1}(X, e)}$ is ample.

ii) The dimension of the obstruction group of the 1-morphism $\zeta: B \rightarrow \overline{\mathcal{M}}_{0,1}(X, e)$ is 0 . In particular there is a discrete valuation ring, $R$, a family of prestable curves, $\pi: \mathcal{B} \rightarrow \operatorname{Spec} R$, and a positive 1-morphism $\zeta_{R}: \mathcal{B} \rightarrow \overline{\mathcal{M}}_{0,1}(X, e)$ such that the geometric closed fiber is $\zeta$, such that the geometric generic fiber $\mathcal{B}_{\eta}$ is smooth, and such that $\zeta_{R}(\mathcal{B})$ is contained in the locus of very stable maps.

Proof. i) By hypothesis, $\zeta$ is positive (respectively very positive). By the relative version of Lemma 3.21, the image of $\zeta$ is in the smooth locus of $\overline{\mathcal{M}}_{0,1}(X, e)$ and there is a short exact sequence,

$$
0 \longrightarrow \sigma^{*} \mathcal{O}_{\Sigma}(\sigma) \longrightarrow \zeta^{*} T_{\overline{\mathcal{M}}_{0,1}(X, e)} \longrightarrow(\mathrm{pr} \circ \zeta)^{*} T_{\overline{\mathcal{M}}_{0,0}(X, e)} \longrightarrow 0 .
$$

By Definition 4.11, parts iv and v, $(\operatorname{pr} \circ \zeta)^{*} T_{\overline{\mathcal{M}}_{0,0}(X, e)}$ is deformation ample and $\sigma^{*} \mathcal{O}_{\Sigma}(\sigma)$ is generated by global sections (respectively deformation ample). Hence $\zeta^{*} T_{\overline{\mathcal{M}}_{0,1}(X, e)}$ is generated by global sections (respectively deformation ample by Lemma 2.10, part ii). Since $B$ is smooth, $\zeta$ is free (respectively very free).

ii) Let $R^{\prime}$ be a discrete valuation ring and let $\pi: \mathcal{B}^{\prime} \rightarrow$ Spec $R^{\prime}$ be a smoothing of $B$, i.e. there is an isomorphism $i: B \rightarrow \mathcal{B}_{0}^{\prime}$ and the generic fiber $\mathcal{B}_{\eta}^{\prime}$ is smooth. For technical reasons it is necessary to compactify $\overline{\mathcal{M}}_{0,1}(X, e)$. Let $j: X \hookrightarrow \bar{X}$ be an open immersion of $X$ into a projective scheme, and let $j_{*}: \overline{\mathcal{M}}_{0,1}(X, e) \hookrightarrow \overline{\mathcal{M}}_{0,1}(\bar{X}, e)$ be the corresponding open immersion of moduli stacks. By [FP95], $\overline{\mathcal{M}}_{0,1}(\bar{X}, e)$ is a proper Deligne-Mumford stack with projective coarse moduli space. Form the $R^{\prime}$-stack, $\mathcal{B}^{\prime} \times \overline{\mathcal{M}}_{0,1}(\bar{X}, e)$. With respect to an ample invertible sheaf on the coarse moduli space, let $\epsilon$ denote the degree of the map $(i, \zeta): B \rightarrow \mathcal{B}^{\prime} \times \overline{\mathcal{M}}_{0,1}(\bar{X}, e)$. Then $(i, \zeta)$ is an object of the Abramovich-Vistoli stack,

$$
\mathcal{K}=\mathcal{K}_{0,0}\left(\mathcal{B}^{\prime} \times \overline{\mathcal{M}}_{0,1}(\bar{X}, e), \epsilon\right) \rightarrow \operatorname{Spec} R^{\prime},
$$

that parametrizes twisted stable maps to $\mathcal{B}^{\prime} \times \overline{\mathcal{M}}_{0,1}(\bar{X}, e)$ over Spec $R^{\prime}$; cf. [AV02]. The stack $\mathcal{K} \rightarrow$ $\operatorname{Spec} R^{\prime}$ is a Deligne-Mumford stack that is proper over Spec $R^{\prime}$, and the coarse moduli space is projective over $\operatorname{Spec} R^{\prime}$. 


\section{RATIONAL CURVES ON HYPERSURFACES, II}

The deformation theory of twisted stable maps is developed in [AV02, § 5], and is exactly analogous to the deformation theory of stable maps. In particular, the obstruction space to the morphism $o: \mathcal{K} \rightarrow \operatorname{Spec} R^{\prime}$ at $[(i, \zeta)]$ is a quotient of $H^{1}\left(B, \zeta^{*} T_{\overline{\mathcal{M}}_{0,1}(X, e)}\right)$. Since $\zeta^{*} T_{\overline{\mathcal{M}}_{0,1}(X, e)}$ is generated by global sections, by Lemma 2.3, part $\mathrm{i}, h^{1}\left(B, \zeta^{*} T_{\overline{\mathcal{M}}_{0,1}(X, e)}\right)=0$. Therefore $o$ is smooth at $[(i, \zeta)]$. By [BLR90, Proposition 2.2.14], there exists an étale morphism of discrete valuation rings, $\operatorname{Spec} R \rightarrow \operatorname{Spec} R^{\prime}$ and a section $s: \operatorname{Spec} R \rightarrow \mathcal{K}$ of $o$. In other words, there exists a family of twisted stable maps over $\operatorname{Spec} R$ whose closed fiber is isomorphic to the pullback of $(i, \zeta)$, i.e.

$$
\left(\pi: \mathcal{B} \longrightarrow \operatorname{Spec} R^{\prime}, \lambda: \mathcal{B} \longrightarrow \operatorname{Spec} R \times \operatorname{Spec} R^{\prime}\left(\mathcal{B}^{\prime} \times \overline{\mathcal{M}}_{0,1}(\bar{X}, e)\right)\right)
$$

Because $B$ is a scheme, the family of curves over $\operatorname{Spec} R$ is a scheme, i.e. $\pi: \mathcal{B} \rightarrow$ Spec $R$ is a morphism of schemes. Because the composition of $(i, \zeta)$ with projection onto the closed fiber of $\mathcal{B}^{\prime}$ is an isomorphism, the composition

$$
\mathcal{B} \stackrel{\lambda}{\longrightarrow} \operatorname{Spec} R \times{ }_{\operatorname{Spec}} R^{\prime}\left(\mathcal{B}^{\prime} \times \overline{\mathcal{M}}_{0,1}(\bar{X}, e)\right) \stackrel{\operatorname{pr}_{\mathcal{B}^{\prime}}}{\longrightarrow} \operatorname{Spec} R \times_{\operatorname{Spec}} R^{\prime} \mathcal{B}^{\prime}
$$

is an isomorphism. Since the image of $(i, \zeta)$ is contained in the open substack $\mathcal{B}^{\prime} \times \overline{\mathcal{M}}_{0,1}(X, e)$, the image of $\lambda$ is contained in the open substack $\operatorname{Spec} R \times_{\operatorname{Spec}} R^{\prime}\left(\mathcal{B}^{\prime} \times \overline{\mathcal{M}}_{0,1}(X, e)\right)$. Putting the pieces together, $\pi: \mathcal{B} \rightarrow$ Spec $R$ is a smoothing of $B$, and $\lambda$ induces a 1-morphism of $R$-stacks, $\zeta_{R}: \mathcal{B} \rightarrow \overline{\mathcal{M}}_{0,1}(X, e)$, such that the closed fiber is $\zeta$.

By Lemma $4.13, \zeta_{R}$ is positive. Since the locus of very stable maps in $\overline{\mathcal{M}}_{0,1}(X, e)$ is open, the image of $\zeta_{R}$ is contained in this locus.

Remark 4.15. The use of the Abramovich-Vistoli stack above is a bit contrived. The most natural stack to use is the Hom stack parametrizing 1-morphisms between two flat, proper, tame DeligneMumford stacks. Martin Olsson has proved existence and some foundational properties of the Hom stack in the generality needed above, but the details have not yet been published.

The following is the main definition of this section.

Definition 4.16. Let $\pi: B \rightarrow T$ be a family of prestable curves of arithmetic genus 0 . An inducting pair of degree $e$ is a pair of 1-morphisms

$$
\left(\zeta_{1}: B \longrightarrow \overline{\mathcal{M}}_{0,1}(X, 1), \bar{\zeta}_{e}: B \longrightarrow \overline{\mathcal{M}}_{0,1}(X, e)\right),
$$

such that

i) $\zeta_{1}$ is very twisting,

ii) $\bar{\zeta}_{e}$ is very positive and the image of $\bar{\zeta}_{e}$ is contained in the locus of very stable maps, and

iii) the morphisms $h_{\zeta_{1}}: B \rightarrow X$ and $h_{\bar{\zeta}_{e}}: B \rightarrow X$ are equal.

Lemma 4.17. Let $\pi: B \rightarrow T$ be a family of prestable curves of arithmetic genus 0 , and let

$$
\left(\zeta_{1}: B \longrightarrow \overline{\mathcal{M}}_{0,1}(X, 1), \bar{\zeta}_{e}: B \longrightarrow \overline{\mathcal{M}}_{0,1}(X, e)\right)
$$

be a pair of 1-morphisms such that $h_{\zeta_{1}}=h_{\bar{\zeta}_{e}}$. Then there is an open subscheme $U_{\text {induct }} \subset T$ with the following property: for every morphism of schemes $f: T^{\prime} \rightarrow T$, the pullback of $\left(\zeta_{1}, \bar{\zeta}_{e}\right)$ is inducting if and only if $f\left(T^{\prime}\right) \subset U$.

Proof. Define $U_{\text {induct }}$ to be the intersection of the open subset $U_{\text {vtwist }} \subset T$ from Lemma 4.6 for $\zeta_{1}$ and the open subset $U_{\text {vpos }} \subset T$ from Lemma 4.13 for $\bar{\zeta}_{e}$.

The final definition of this section is the following. 


\section{J. HARRIS AND J. STARR}

Definition 4.18. Let $\left(\pi: B \rightarrow T, \bar{\zeta}_{e}: B \rightarrow \overline{\mathcal{M}}_{0,1}(X, e)\right)$ be a very positive family whose image is contained in the locus of very stable maps. The 1-morphism $\bar{\zeta}_{e}$ is inductable if there exists a surjective étale morphism $u: T^{\prime} \rightarrow T$ and a morphism $\zeta_{1}: u^{*} B \rightarrow \overline{\mathcal{M}}_{0,1}(X, 1)$ such that

i) $h_{\zeta_{1}}=u^{*} h_{\bar{\zeta}_{e}}$, and

ii) $\left(\zeta_{1}, \bar{\zeta}_{e}\right)$ is an inducting pair.

Lemma 4.19. Let $\left(\pi: B \rightarrow T, \bar{\zeta}_{e}: B \rightarrow \overline{\mathcal{M}}_{0,1}(X, e)\right)$ be a very free family. There is an open subscheme $U_{\text {i-able }} \subset T$ with the following property: for every morphism of schemes $f: T^{\prime} \rightarrow T$, the pullback $\left(f^{*} \pi: f^{*} B \rightarrow T^{\prime}, f^{*} \bar{\zeta}_{e}: f^{*} B \rightarrow \overline{\mathcal{M}}_{0,1}(X, e)\right)$ is inductable if and only if $f\left(T^{\prime}\right) \subset U_{\text {i-able }}$.

Proof. Apply Proposition 4.8 to

$$
\xi:=\left(\pi: B \rightarrow T, h_{\bar{\zeta}_{e}}: B \rightarrow X\right) .
$$

\section{The induction argument}

In this section it is proved that if $X$ satisfies Hypotheses 1.5, 1.6, 1.7, and 4.10, and if there exists an inductable 1-morphism $\bar{\zeta}_{e}: B \rightarrow \overline{\mathcal{M}}_{0,1}(X, e)$, then there exists an inductable 1-morphism $\bar{\zeta}_{e+1}: B \rightarrow \overline{\mathcal{M}}_{0,1}(X, e+1)$. The basic idea is, given an inducting pair $\left(\zeta_{1}, \bar{\zeta}_{e+1}\right)$, to form the family of 'connected sums', i.e. the family of reducible curves obtained by gluing the families $\zeta_{1}$ and $\bar{\zeta}_{e+1}$ along the two sections. However, this is a family of unpointed curves, and an inducting family is a family of pointed curves. By hypothesis, the section of $\bar{\zeta}_{e}, \bar{\sigma}: B \rightarrow \bar{\Sigma}$, is such that the Cartier divisor $\bar{\sigma}(B) \subset \bar{\Sigma}$ moves. A general member of the linear system $|\bar{\sigma}(B)|$ is the image of a section, $\sigma^{\prime}: B \rightarrow \bar{\Sigma}$. Except at finitely many points of $B$, the sections $\bar{\sigma}$ and $\sigma^{\prime}$ are disjoint. Away from these points, the section of the family of reducible curves is taken to be $\sigma^{\prime}$. The stable limit over the finitely many points of $B$ is obtained by blowing-up $\bar{\Sigma}$ along the zero-dimensional scheme $\bar{\sigma}(B) \cap \sigma^{\prime}(B)$. Unfortunately, the resulting family of pointed, reducible curves is no longer very positive.

To make the resulting family very positive, the family is altered by a modification.

\subsection{Modification}

Definition 5.1. An input triple is a triple $I=\left(\zeta, L, \sigma_{i}\right)$ where

i) $\zeta=\left(\left(\Sigma, \sigma_{1}, \ldots, \sigma_{r}\right), g: \Sigma \rightarrow X\right)$ is an $r$-pointed stable map to $X$ of arithmetic genus $g$,

ii) $L \subset \Sigma$ is an irreducible component that is smooth, and

iii) $\sigma_{i} \in L$ is a marked point.

Let $I=\left(\zeta, L, \sigma_{i}\right)$ be an input triple. Denote by $M \subset \Sigma$ the union of all irreducible components other than $L$. Denote by $R=\left(\rho_{1}, \ldots, \rho_{c}\right)$ the intersection $L \cap M$, denote by $S=\left(\sigma_{j_{1}}, \ldots, \sigma_{j_{d}}\right)$ the marked points that are contained in $L$ other than $\sigma_{i}$, and denote by $S^{\prime}=\left(\sigma_{k_{1}}, \ldots, \sigma_{k_{e}}\right)$ the marked points that are contained in $M$.

Denote by $\Delta: L \rightarrow L \times L$ the diagonal, and denote by $u: \Lambda \rightarrow L \times L$ the blowing-up of $L \times L$ along $\Delta(R \cup S)$. For each closed point $\lambda \in R \cup S$, denote by $F_{\lambda} \subset \Lambda$ the proper transform of $L \times\{\lambda\} \subset L \times L$. Denote by $F_{\Delta} \subset \Lambda$ the proper transform of $\Delta(L) \subset L \times L$. For each $\lambda \in R \cup S$, there exists a unique section of $\operatorname{pr}_{1} \circ u: \Lambda \rightarrow L, \sigma_{I, \lambda}: L \rightarrow \Lambda$, such that $F_{\lambda}=\sigma_{I, \lambda}(L)$. Also there is a section of $\operatorname{pr}_{1} \circ u, \sigma_{I, \Delta}$, such that $\sigma_{I, \Delta}(L)=F_{\Delta}$.

Consider the projection $\operatorname{pr}_{1}: L \times M \rightarrow L$. For each closed point $\lambda \in R \cup S^{\prime}$ there is a unique section of $\operatorname{pr}_{1}, \sigma_{I, \lambda}^{\prime}$, such that $\sigma_{I, \lambda}^{\prime}(L)=L \times\{\lambda\}$. Let $\Sigma_{I}$ be the simple normal crossing surface containing $\Lambda$ and $L \times M$ that is obtained by identifying the divisor $\sigma_{I, \lambda}(L) \subset \Lambda$ with $\sigma_{I, \lambda}^{\prime}(L) \subset L \times M$ for each $\lambda \in R$. The divisors are identified by $\sigma_{I, \lambda}(t) \leftrightarrow \sigma_{I, \lambda}^{\prime}(t)$ for each $t \in L$. There is a unique 


\section{RATIONAL CURVES ON HYPERSURFACES, II}

morphism $p_{I}: \Sigma_{I} \rightarrow L$ whose restriction to $\Lambda$ is $\operatorname{pr}_{1} \circ u$ and whose restriction to $L \times M$ is $\operatorname{pr}_{1}$. There is a unique morphism $g_{I}: \Sigma_{I} \rightarrow X$ whose restriction to $\Lambda$ is $\left.g\right|_{L} \circ \mathrm{pr}_{2} \circ u$ and whose restriction to $L \times M$ is $\left.g\right|_{M} \circ \operatorname{pr}_{2}$. For each integer $j=1, \ldots, r$ such that $j \neq i$, there exists a section of $p_{I}$, $\sigma_{I, j}: L \rightarrow \Sigma_{I}$, given by

$$
\sigma_{I, j}= \begin{cases}\sigma_{I, \lambda}, & \lambda=\sigma_{j} \in S, \\ \sigma_{I, \lambda}^{\prime}, & \lambda=\sigma_{j} \in S^{\prime} .\end{cases}
$$

Also there exists a section $\sigma_{I, i}: L \rightarrow \Sigma_{I}$ given by $\sigma_{I, i}=\sigma_{I, \Delta}$. By construction, the sections $\sigma_{I, j}$ are pairwise disjoint. Hence the datum

$$
\zeta_{I}=\left(\left(p_{I}: \Sigma_{I} \rightarrow L, \sigma_{I, 1}, \ldots, \sigma_{I, r}\right), g_{I}: \Sigma_{I} \rightarrow X\right)
$$

is a family of $r$-pointed stable maps to $X$ of arithmetic genus $g$. The fiber over $\sigma_{i} \in L$ is canonically isomorphic to $\zeta$.

Definition 5.2. The family of stable maps, $\zeta_{I}$, is the modification associated to $I$.

The relevance of this construction is the following. Let $B$ be a prestable curve and let

$$
\zeta=\left(\left(p: \Sigma \rightarrow B, \sigma_{1}, \ldots, \sigma_{r}\right), g: \Sigma \rightarrow X\right)
$$

be a family of $r$-pointed stable maps to $X$ of arithmetic genus $g$. Let $b \in B$ be a smooth point, let $L \subset \Sigma_{b}$ be an irreducible component that is smooth, and let $\sigma_{i}(b) \in L$ be a marked point. Then $I=\left(\zeta_{b}, L, \sigma_{i}(b)\right)$ is an input triple. Let $\zeta_{I}$ be the modification associated to $I$. The fiber $\zeta_{I, \sigma_{i}(b)}$ is canonically isomorphic to the fiber $\zeta_{b}$. Let $\widetilde{B}$ be the prestable curve containing $B$ and $L$ obtained by identifying $b \in B$ with $\sigma_{i}(b) \in L$. There is a unique family of $r$-pointed stable maps to $X$ of arithmetic genus $g$,

$$
\widetilde{\zeta}=\left(\left(\widetilde{p}: \widetilde{\Sigma} \rightarrow \widetilde{B}, \widetilde{\sigma}_{1}, \ldots, \widetilde{\sigma}_{r}\right), \widetilde{g}: \widetilde{\Sigma} \rightarrow X\right),
$$

whose restriction to $B$ is $\zeta$ and whose restriction to $L$ is $\zeta_{I}$.

Definition 5.3. The family of stable maps, $\widetilde{\zeta}$, is the modification of $\zeta$ associated to $\left(b, L, \sigma_{i}(b)\right)$.

Let $I=\left(\zeta, L, \sigma_{i}\right)$ be an input triple. Denote by $\zeta_{\widehat{\sigma}_{i}}$ the $(r-1)$-pointed stable map obtained by omitting the marked point $\sigma_{i}$.

Lemma 5.4. Let $I=\left(\zeta, L, \sigma_{i}\right)$ be an input triple.

i) The dimension of the obstruction space of $\zeta_{\widehat{\sigma}_{i}}$ is 0 and, for each closed point $\lambda \in R$, there exists a first-order deformation of $\zeta_{\widehat{\sigma}_{i}}$ that smoothes the node $\lambda$ if and only if the same is true for $\zeta$.

ii) For every closed point $\lambda \in L$, the dimension of the obstruction space of $\zeta_{I, \lambda}$ is 0 if and only if both the dimension of the obstruction space of $\zeta_{\widehat{\sigma}_{i}}$ is 0 and for each closed point $\lambda \in R$ there exists a first-order deformation of $\zeta_{\widehat{\sigma_{i}}}$ that smoothes the node $\lambda$.

iii) If the equivalent conditions of part ii are satisfied then there is an exact sequence,

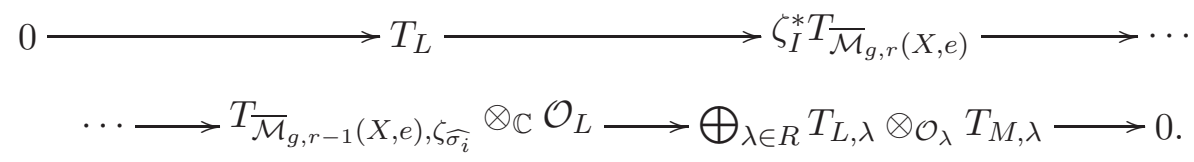

iv) If the equivalent conditions of part iii are satisfied, if $L$ has genus 0 , and if the map

$$
T_{\overline{\mathcal{M}}_{g, r-1}(X, e), \zeta_{\widehat{\sigma_{i}}}} \longrightarrow \bigoplus_{\lambda \in R} T_{L, \lambda} \otimes T_{M, \lambda}
$$

is surjective, then $h^{1}\left(L, \zeta_{I}^{*} T_{\overline{\mathcal{M}}_{g, r}(X, e)}\right)=0$. 


\section{J. HARRIS AND J. STARR}

Proof. Part i follows by Lemma 3.22. Parts ii and iii follow by Lemmas 3.18, 3.19, 3.21 and 3.22. If $L$ has genus 0 , then $T_{L}$ is generated by global sections. So part iv follows from the long exact sequences in cohomology associated to the exact sequence in part ii and by Lemma 2.3 part i.

Let $\zeta$ be a stable map of arithmetic genus 0 and let $I=\left(\zeta, L, \sigma_{i}\right)$ be an input pair.

Lemma 5.5. If the dimension of the obstruction group of $\left.g\right|_{L}: L \rightarrow X$ is 0 , and if the pullback of $T_{X}$ by $\left.g\right|_{M}: M \rightarrow X$ is generated by global sections, then for every closed point $\lambda \in L$, the dimension of the obstruction space of $\zeta_{I, \lambda}$ is 0 , and $h^{1}\left(L, \zeta_{I}^{*} T_{\overline{\mathcal{M}}_{0, r}(X, e)}\right)=0$.

Proof. By Lemmas 3.17, 3.21 and 3.22, the family of pointed stable curves,

$$
\left(\left(\mathrm{pr}_{1} \circ u: \Lambda \longrightarrow L, \sigma_{\lambda}, \sigma_{\Delta}\right),\left.g\right|_{L} \circ \mathrm{pr}_{2} \circ u: \Lambda \longrightarrow X\right),
$$

is such that for each point of $L$ the dimension of the obstruction space is 0 . Consider the families obtained by successively gluing on the connected components of $L \times M$. Applying Lemma 3.29 to each of these families, the hypotheses of Lemma 5.4, part iv are satisfied for $\zeta_{I}$.

Let $B$ be a prestable curve of arithmetic genus 0 , and let

$$
\zeta=\left(\left(p: \Sigma \rightarrow B, \sigma_{1}, \ldots, \sigma_{r}\right), g: \Sigma \rightarrow X\right)
$$

be a family of $r$-pointed stable maps of arithmetic genus 0 . Let $b \in B$ be a smooth point, let $L \subset \Sigma_{b}$ be an irreducible component that is not contracted by $g$, and let $\sigma_{i}(b) \in L$ be a marked point. Let $M$ be the union of all irreducible components of $\Sigma_{b}$ other than $L$.

LEMmA 5.6. If for every point $b^{\prime} \in B$ the dimension of the obstruction group of $\zeta_{b^{\prime}}$ is 0 , if $\zeta^{*} T_{\overline{\mathcal{M}}_{0, r}(X, e)}$ is generated by global sections, if the dimension of the obstruction group of $\left.g\right|_{L}: L \rightarrow X$ is 0 , and if the pullback of $T_{X}$ by $\left.g\right|_{M}: M \rightarrow X$ is generated by global sections, then for every point $b^{\prime} \in \widetilde{B}$ the dimension of the obstruction group of $\widetilde{\zeta}_{b^{\prime}}$ is 0 and $h^{1}\left(\widetilde{B}, \widetilde{\zeta}^{*} T_{\overline{\mathcal{M}}_{0, r}(X, e)}\right)=0$.

Therefore the dimension of the obstruction group of $\widetilde{\zeta}: \widetilde{B} \rightarrow \overline{\mathcal{M}}_{0, r}(X, e)$ is 0 . In particular there is a discrete valuation ring, $R$, a family of prestable curves, $\pi: \mathcal{B} \rightarrow \operatorname{Spec} R$, and a 1-morphism $\zeta_{R}: \mathcal{B} \rightarrow \overline{\mathcal{M}}_{0, r}(X, e)$ such that the geometric closed fiber is $\widetilde{\zeta}$ and such that the geometric generic fiber $\mathcal{B}_{\eta}$ is smooth.

Proof. The hypotheses of Lemma 5.5 are satisfied for the modification associated to $I=\left(\zeta_{b}, L, \sigma_{i}(b)\right)$, $\widetilde{\zeta}_{L}: L \rightarrow \overline{\mathcal{M}}_{0, r}(X, e)$. Hence the dimension of the obstruction group of $\widetilde{\zeta}$ at each closed point of $L$ is 0 and $h^{1}\left(L, \widetilde{\zeta}_{L}^{*} T \overline{\mathcal{M}}_{0, r}(X, e)=0\right.$. There is a short exact sequence of coherent sheaves on $\widetilde{B}$,

$$
0 \longrightarrow \zeta^{*} T_{\overline{\mathcal{M}}_{0, r}(X, e)}(-b) \longrightarrow \widetilde{\zeta}^{*} T_{\overline{\mathcal{M}}_{0, r}(X, e)} \longrightarrow \widetilde{\zeta}_{L}^{*} T_{\overline{\mathcal{M}}_{0, r}(X, e)} \longrightarrow 0 .
$$

By Lemma 2.3, part ii, $h^{1}\left(B, \zeta^{*} T_{\overline{\mathcal{M}}_{0, r}(X, e)}(-b)\right)=0$. Hence by the long exact sequence of cohomology associated to the short exact sequence above, $h^{1}\left(\widetilde{B}, \widetilde{\zeta}^{*} T_{\overline{\mathcal{M}}_{0, r}(X, e)}\right)=0$.

The second part of the lemma follows by the same proof as in Lemma 4.14, part ii.

\subsection{The induction argument}

Notation 5.7. The notation for the generators of the group of $\mathbb{Q}$-Cartier divisor classes on $\overline{\mathcal{M}}_{0,1}\left(\mathbb{P}^{n}, e\right)$ is taken from [Pan99].

i) For each integer $0 \leqslant i \leqslant\lfloor e\rfloor$ denote by $\Delta_{i}$, or sometimes $\Delta_{i, e-i}$, the class of the $\mathbb{Q}$-Cartier divisor that is the closure of the locus parametrizing embedded curves that have one irreducible component of degree $i$, one irreducible component of degree $e-i$, and where the marked point is on the irreducible component of degree $i$. 


\section{RATIONAL CURVES ON HYPERSURFACES, II}

ii) Denote by $\mathcal{L}$ the first Chern class of ev $^{*} \mathcal{O}_{\mathbb{P}^{n}}(1)$.

iii) Denote by $\mathcal{H}$ the class of the $\mathbb{Q}$-Cartier divisor parametrizing stable maps $g: \Sigma \rightarrow \mathbb{P}^{n}$ such that $g(\Sigma) \cap \Lambda \neq \emptyset$, where $\Lambda \subset \mathbb{P}^{n}$ is a fixed linear $\mathbb{P}^{n-2}$.

Let $B$ be a prestable curve of genus 0 . Let $\zeta=((p: \Sigma \rightarrow B, \sigma), g: \Sigma \rightarrow X)$ be a family of stable maps of genus 0 and degree $e>1$. Denote by $h: B \rightarrow X$ the composition $g \circ \sigma$.

Definition 5.8. The 1-morphism $\zeta: B \rightarrow \overline{\mathcal{M}}_{0,1}(X, e)$ is typical if:

i) the curve $B$ is smooth,

ii) for every closed point $b \in B$, the obstruction group of $\zeta_{b}$ has dimension 0 ,

iii) for every point $b \in B$, the stable map $\zeta_{b}$ has only the trivial automorphism,

iv) for every integer $j=1, \ldots, e-1$, the preimage $\zeta^{-1}\left(\Delta_{j}\right)$ consists of finitely many points, and for each $b \in \zeta^{-1}\left(\Delta_{j}\right)$, the curve $\Sigma_{b}$ has only two irreducible components,

v) for every point $b \in \zeta^{-1}\left(\Delta_{1}\right), \Sigma_{b}$ is a union of two irreducible components $L \cup M$ with $\sigma(b) \in L$ such that $\left.g\right|_{L}: L \rightarrow X$ is an isomorphism to a twistable line.

Lemma 5.9. Let $\pi: B \rightarrow T$ be a family of prestable curves of arithmetic genus 0 and let $\zeta$ : $B \rightarrow \overline{\mathcal{M}}_{0,1}(X, e)$ be a 1-morphism. There is an open subscheme $U_{\text {typ }} \subset T$ with the following property: for every morphism of schemes $f: T^{\prime} \rightarrow T$, the pullback family $f^{*} \pi: f^{*} B \rightarrow T^{\prime}$ and $f^{*} \zeta: f^{*} B \rightarrow \overline{\mathcal{M}}_{0,1}(X, e)$ is typical if and only if $f\left(T^{\prime}\right) \subset U_{\text {typ }}$.

Proof. Each of the conditions in Definition 5.8 is clearly an open condition.

Lemma 5.10. If $X$ satisfies Hypotheses 1.5, 1.6, 1.7, and 4.10, if for every closed point $b \in B$ the obstruction group of $\zeta_{b}$ has dimension 0 , and if $\zeta^{*} T_{\overline{\mathcal{M}}_{0,1}(X, e)}$ is generated by global sections, then there exists a discrete valuation ring, $R$, a family of prestable curves, $\pi: \mathcal{B} \rightarrow$ Spec $R$, and a 1-morphism $\zeta_{R}: \mathcal{B} \rightarrow \overline{\mathcal{M}}_{0,1}(X, e)$ such that the geometric closed fiber is $\zeta$, such that the geometric generic fiber $\mathcal{B}_{\eta}$ is smooth, and such that $\left(\zeta_{R}\right)_{\eta}$ is typical.

Proof. By the same argument as in the proof of Lemma 4.13, there exists a family $\zeta_{R}: \mathcal{B} \rightarrow$ $\overline{\mathcal{M}}_{0,1}(X, e)$ such that $\mathcal{B}_{\eta}$ is smooth. The condition that for every point the obstruction group has dimension 0 is stable under generization, so it also holds for $\left(\zeta_{R}\right)_{\eta}$. Similarly, $\left(\zeta_{R}\right)_{\eta}^{*} T_{\overline{\mathcal{M}}_{0,1}(X, e)}$ is generated by global sections. By [Kol96, Proposition 3.7], the family $\zeta_{R}$ may be chosen so that $\left(\zeta_{R}\right)\left(B_{\eta}\right)$ is disjoint from any given finite collection of closed substacks of codimension at least 2 . By Hypothesis 1.5, the locus of stable maps such that the domain curve has three or more irreducible components has codimension at least 2 . Let $Z \subset \Delta_{i}$ be a closed substack whose image in the coarse moduli space of $\Delta_{i}$ is an ample divisor. Again by Hypothesis 1.5, $Z$ has codimension 2 in $\overline{\mathcal{M}}_{0,1}(X, e)$. So $\left(\zeta_{R}\right)\left(B_{\eta}\right)$ does not intersect $Z$, and therefore it is not contained in $\Delta_{i}$. By Hypothesis 1.7 , the locus of stable maps that have a nontrivial automorphism has codimension at least 2. Finally, by Hypotheses 1.6 and 4.10, the locus in $\Delta_{1}$ parametrizing stable maps $g: L \cup M \rightarrow X$ such that $\left.g\right|_{L}: L \rightarrow X$ is an isomorphism to a twistable line is a dense open in $\Delta_{1}$. In particular the complement in $\Delta_{1}$ is a closed substack that has codimension 2 in $\overline{\mathcal{M}}_{0,1}(X, e)$. Therefore $\left(\zeta_{R}\right)_{\eta}$ is typical.

By Hypothesis 4.10, a general line $L \subset X$ is twistable. Denote by

$$
\zeta_{L}=\left(\left(p_{L}: \Sigma_{L} \rightarrow L, \sigma_{L}\right), g_{L}: \Sigma_{L} \rightarrow X\right)
$$

a twisting family such that $h_{L}: L \rightarrow X$ is the inclusion. By Remark 4.4, part iv, the degree of $g_{L}$ is either 1 or 2, i.e. either $g_{L}: \Sigma_{L} \rightarrow X$ is a birational map to a linear $\mathbb{P}^{2}$ in $X$ (obtained by blowing-up a point on the $\mathbb{P}^{2}$ ), or $g_{L}$ is an isomorphism of $\Sigma_{L}$ to a nonsingular quadric surface in $X$. 


\section{J. HARRIS AND J. STARR}

Definition 5.11. Let $X$ be a variety that satisfies Hypotheses 1.6 and 4.10. The variety $X$ is planar type if for a general line $L$ there exists a twisting family such that the degree of $g_{L}$ is 1 . The variety is quadric type if it is not planar type.

Remark 5.12. It is not hard to prove that, if $X$ is planar type, then there exists an integer $r \geqslant 2$ such that, for a general point $x \in X$, the set of lines in $X$ that contain $x$ sweep out a linear $\mathbb{P}^{r}$. Let $Q$ denote the irreducible component of the Hilbert scheme of the $\mathbb{P}^{r}$ in $X$ that contains the $\mathbb{P}^{r}$ constructed in this way. Then $X$ is birationally a $\mathbb{P}^{r}$-bundle over $Q$ and, with its natural Plücker embedding, $Q$ is not uniruled by lines. If $X$ satisfies Hypothesis 1.5, then $Q$ is not uniruled by rational curves of any degree. If also $X$ contains a very twistable curve, then $Q$ is a point and $X$ is isomorphic to $\mathbb{P}^{r}$ (not merely birational to $\mathbb{P}^{r}$ ). Thus, in what follows, $X$ will usually be of quadric type.

Let $\left(\zeta_{1}, \bar{\zeta}_{e}\right)$ be an inducting pair denoted by

$$
\begin{aligned}
& \zeta_{1}=((p: \Sigma \rightarrow B, \sigma), g: \Sigma \rightarrow X), \\
& \bar{\zeta}_{e}=((\bar{p}: \bar{\Sigma} \rightarrow B, \bar{\sigma}), \bar{g}: \bar{\Sigma} \rightarrow X) .
\end{aligned}
$$

Denote

$$
\begin{aligned}
& \delta=\operatorname{deg}\left(\zeta_{1}^{*}(2 \mathcal{L}-\mathcal{H})\right) \\
& \bar{\delta}=\operatorname{deg}\left(\bar{\zeta}_{e}^{*}\left(\frac{2}{e} \mathcal{L}-\frac{1}{e^{2}} \mathcal{H}-\sum_{i=1}^{e-1} \frac{(e-i)^{2}}{e^{2}} \Delta_{i}\right)\right) .
\end{aligned}
$$

By $\left[\right.$ Pan99, Lemma 2.2.2], $\operatorname{deg}\left(\sigma^{*} \mathcal{O}_{\Sigma}(\sigma(B))\right)=\delta$ and $\operatorname{deg}\left(\bar{\sigma}^{*} \mathcal{O}_{\bar{\Sigma}}(\bar{\sigma}(B))\right)=\bar{\delta}$. In particular, both $\delta$ and $\bar{\delta}$ are nonnegative integers, and $\bar{\delta}$ is positive by Definition 4.11 .

Theorem 5.13. For each integer $d=1, \ldots, \bar{\delta}$, there exists an inducting pair $\left(\xi_{d, 1}, \bar{\xi}_{d, e+1}\right)$ such that $\bar{\xi}_{d, e+1}$ is typical and such that the following conditions are satisfied,

$$
\left\{\begin{array}{rlrl}
\operatorname{deg}\left(\bar{\xi}_{d, e+1}^{*} \mathcal{H}\right) & =\operatorname{deg}\left(\bar{\zeta}_{e}^{*} \mathcal{H}\right)+\operatorname{deg}\left(\zeta_{1}^{*} \mathcal{H}\right), & & \\
\operatorname{deg}\left(\xi_{d, 1}^{*} \mathcal{H}\right) & =\operatorname{deg}\left(\zeta_{1}^{*} \mathcal{H}\right)+d, & & \text { if } X \text { is planar type } \\
\operatorname{deg}\left(\xi_{d, 1}^{*} \mathcal{H}\right) & =\operatorname{deg}\left(\zeta_{1}^{*} \mathcal{H}\right)+2 d, & & \text { if } X \text { is quadric type } \\
\operatorname{deg}\left(\xi_{d, 1}^{*} \mathcal{L}\right) & =\operatorname{deg}\left(\zeta_{1}^{*} \mathcal{L}\right)+d, & & \\
\operatorname{deg}\left(\bar{\xi}_{d, e+1}^{*} \mathcal{L}\right) & =\operatorname{deg}\left(\bar{\zeta}_{e}^{*} \mathcal{L}\right)+d, & & i=2, \ldots, e-1 \\
\operatorname{deg}\left(\bar{\xi}_{d, e+1}^{*} \Delta_{i}\right) & =\operatorname{deg}\left(\bar{\zeta}_{e}^{*} \Delta_{i-1}\right), & & \text { if } i>1 \\
\operatorname{deg}\left(\bar{\xi}_{d, e+1}^{*} \Delta_{e}\right) & =\operatorname{deg}\left(\bar{\zeta}_{e}^{*} \Delta_{e-1}\right)+\delta+d, & & \text { if } i>1 \\
\operatorname{deg}\left(\bar{\xi}_{d, e+1}^{*} \Delta_{1}\right) & =\bar{\delta}-d, & & \text { if } i=1 . \\
\operatorname{deg}\left(\bar{\xi}_{d, 2}^{*} \Delta_{1}\right) & =\delta+\bar{\delta}, &
\end{array}\right.
$$

The families are denoted

$$
\begin{aligned}
\bar{\xi}_{d, e+1} & =\left(\left(\bar{p}_{d, e+1}: \bar{\Sigma}_{d, e+1} \rightarrow B, \bar{\sigma}_{d, e+1}\right), \bar{g}_{d, e+1}: \bar{\Sigma}_{d, e+1} \rightarrow X\right), \\
\xi_{d, 1} & =\left(\left(p_{d, 1}: \Sigma_{d, 1} \rightarrow B, \sigma_{d, 1}\right), g_{d, 1}: \Sigma_{d, 1} \rightarrow X\right) .
\end{aligned}
$$

Also $\bar{h}_{d, e+1}$ denotes $\bar{g}_{d, e+1} \circ \bar{\sigma}_{d, e+1}$ and $h_{d, 1}$ denotes $g_{d, 1} \circ \sigma_{d, 1}$.

Proof. By Lemma 4.14, part ii and Lemma 4.19, it suffices to consider the case that $B$ is smooth. By Lemma 4.14, part i, $\bar{\zeta}_{e}: B \rightarrow \overline{\mathcal{M}}_{0,1}(X, e)$ is very free. Hence, by the same argument as in the proof of Lemma 4.14, part ii and [Kol96, Proposition 3.7], it suffices to consider the case that $\bar{\zeta}_{e}\left(B_{e}\right)$ is in general position, i.e. for any finite collection of codimension- 2 subvarieties $\left(Z_{\alpha} \mid \alpha=1, \ldots, M\right)$ 


\section{RATIONAL CURVES ON HYPERSURFACES, II}

and any finite collection of divisors $\left(D_{\beta} \mid \beta=1, \ldots, N\right), \bar{\zeta}_{e}\left(B_{e}\right)$ is disjoint from each $Z_{\alpha}$ and has zero-dimensional intersection with each $D_{\beta}$. The proof is long and is split up into a number of families and claims.

The family $\zeta^{(1)}$. Denote by $\Sigma^{(1)}$ the surface containing $\Sigma$ and $\bar{\Sigma}$ obtained by identifying the divisor $\sigma(B) \subset \Sigma$ and $\bar{\sigma}(B) \subset \bar{\Sigma}$ via $\sigma(b) \leftrightarrow \bar{\sigma}(b)$. Denote by $p^{(1)}: \Sigma^{(1)} \rightarrow B$ the unique morphism whose restriction to $\Sigma$ is $p$ and whose restriction to $\bar{\Sigma}$ is $\bar{p}$. Denote by $g^{(1)}: \Sigma^{(1)} \rightarrow X$ the unique morphism whose restriction to $\Sigma$ is $g$ and whose restriction to $\bar{\Sigma}$ is $\bar{g}$. Then $\zeta^{(1)}=\left(p^{(1)}: \Sigma^{(1)} \rightarrow B\right.$, $\left.g^{(1)}: \Sigma^{(1)} \rightarrow X\right)$ is a family of stable maps in the boundary divisor $\Delta_{1} \subset \overline{\mathcal{M}}_{0,0}(X, e+1)$. Denote by $\tau$ the stable $A$-graph with two vertices $v$ and $\bar{v}$ of degree 1 and $e$ respectively, with no tails, and with one edge connecting $v$ and $\bar{v}$. Then $\zeta^{(1)}$ factors through the canonical 1-morphism of Behrend-Manin $\operatorname{stacks} \overline{\mathcal{M}}(X, \tau) \rightarrow \overline{\mathcal{M}}_{0,0}(X, e+1)$.

CLAIM 5.14. The pullback $\left(\zeta^{(1)}\right)^{*} T_{\overline{\mathcal{M}}_{0,0}(X, e)}$ is ample.

By Lemma 3.28, there is a short exact sequence,

$$
0 \longrightarrow \zeta_{1}^{*} T_{\mathrm{ev}} \longrightarrow\left(\zeta^{(1)}\right)^{*} T_{\overline{\mathcal{M}}(X, \tau)} \longrightarrow \bar{\zeta}_{e}^{*} T_{\overline{\mathcal{M}}_{0,1}(X, e)} \longrightarrow 0 .
$$

Since $\zeta_{1}$ is very twisting, by Definition $4.3, \zeta_{1}^{*} T_{\mathrm{ev}}$ is ample. Since $\bar{\zeta}_{e}$ is very positive, by Lemma 4.14 , $\bar{\zeta}_{e}^{*} T_{\overline{\mathcal{M}}_{0,1}(X, e)}$ is ample. Hence $\left(\zeta^{(1)}\right)^{*} T_{\overline{\mathcal{M}}(X, \tau)}$ is ample. By Lemma 3.29 , there is a short exact sequence,

$$
0 \longrightarrow\left(\zeta^{(1)}\right)^{*} T_{\overline{\mathcal{M}}(X, \tau)} \longrightarrow\left(\zeta^{(1)}\right)^{*} T_{\overline{\mathcal{M}}_{0,0}(X, e+1)} \longrightarrow \sigma^{*} \mathcal{O}_{\Sigma}(\sigma) \otimes \bar{\sigma}^{*} \mathcal{O}_{\bar{\Sigma}}(\bar{\sigma}) \longrightarrow 0 .
$$

By Definitions 4.3 and 4.11, both $\sigma^{*} \mathcal{O}_{\Sigma}(\sigma)$ and $\bar{\sigma}^{*} \mathcal{O}_{\bar{\Sigma}}(\bar{\sigma})$ are ample. Therefore the tensor product is ample, and $\left(\zeta^{(1)}\right) * T_{\overline{\mathcal{M}}_{0,0}(X, e+1)}$ is ample.

The family $\zeta^{(2)}$. The family $\zeta^{(1)}$ cannot be an inductable family, because it is a family of unpointed curves rather than 1-pointed curves. The next approximation to $\xi_{d, e+1}$ 'adds' a marked section to $\zeta^{(1)}$. The self-intersection of $\bar{\sigma}(B) \subset \bar{\Sigma}$ is the degree of the invertible sheaf $\bar{\sigma}^{*} \mathcal{O}_{\bar{\Sigma}}(\bar{\sigma}(B))$, which is also

$$
\bar{\delta}=\operatorname{deg}\left(2 \bar{\zeta}_{e}^{*} \mathcal{L}-\bar{\zeta}_{e}^{*} \mathcal{H}\right)
$$

Let $\varsigma: B \rightarrow \bar{\Sigma}$ be a section such that $\varsigma(B) \subset \bar{\Sigma}$ is a general member of the linear system $|\bar{\sigma}(B)|$. Because $\bar{\sigma}^{*} \mathcal{O}_{\bar{\Sigma}}(\bar{\sigma}(B))$ is generated by global sections, there exists $\varsigma$ such that $\varsigma(B)$ has only transverse intersections with $\bar{\sigma}(B)$. Denote the points of intersection by $q_{1}, \ldots, q_{\bar{\delta}} \in \bar{\Sigma}$. Denote by $w: \bar{\Sigma}^{(2)} \rightarrow \bar{\Sigma}$ the blowing-up of $\bar{\Sigma}$ at the points $q_{1}, \ldots, q \bar{\delta}_{\bar{\delta}}$. Let $\bar{p}^{(2)}: \bar{\Sigma}^{(2)} \rightarrow B$ denote the projection $\bar{p} \circ w$. Let $\bar{g}^{(2)}$ denote $\bar{g} \circ w$. Let $\bar{\sigma}^{(2)}: B \rightarrow \bar{\Sigma}^{(2)}$ and $\varsigma^{(2)}: B \rightarrow \bar{\Sigma}^{(2)}$ denote the proper transforms of $\bar{\sigma}$ and $\varsigma$ respectively. Notice that $\bar{\sigma}^{(2)}(B)$ and $\varsigma^{(2)}(B)$ are disjoint by construction. So the data

$$
\bar{\zeta}_{e}^{(2)}=\left(\left(\bar{p}^{(2)}: \bar{\Sigma}^{(2)} \rightarrow B, \bar{\sigma}^{(2)}, \varsigma^{(2)}\right), \bar{g}^{(2)}: \bar{\Sigma}^{(2)} \rightarrow X\right)
$$

is a family of stable 2-pointed maps, i.e. a 1-morphism $\bar{\zeta}_{e}^{(2)}: B \rightarrow \overline{\mathcal{M}}_{0,2}(X, e)$.

CLAim 5.15. The pullback $\left(\bar{\zeta}_{e}^{(2)}\right)^{*} T_{\overline{\mathcal{M}}_{0,2}(X, e)}$ is generated by global sections.

By Lemmas 3.16, 3.21 and 3.22, there is a short exact sequence:

$$
0 \longrightarrow\left(\varsigma^{(2)}\right)^{*} \mathcal{O}_{\bar{\Sigma}^{(2)}}\left(\varsigma^{(1)}(B)\right) \longrightarrow\left(\bar{\zeta}_{e}^{(2)}\right)^{*} T_{\overline{\mathcal{M}}_{0,2}(X, e)} \longrightarrow \bar{\zeta}_{e}^{*} T_{\overline{\mathcal{M}}_{0,1}(X, e)} \longrightarrow 0 .
$$




\section{J. HARRIS AND J. STARR}

By construction, the self-intersection of $\varsigma^{(2)}(B) \subset \bar{\Sigma}^{(2)}$ is 0, i.e. $\left(\varsigma^{(2)}\right)^{*} \mathcal{O}_{\bar{\Sigma}^{(2)}}\left(\varsigma^{(2)}(B)\right)$ is the trivial invertible sheaf $\mathcal{O}_{B}$. By hypothesis $\bar{\zeta}_{e}^{*} T_{\overline{\mathcal{M}}_{0,1}(X, e)}$ is generated by global sections. Therefore by Lemma 2.3, part iii, $\left(\bar{\zeta}_{e}^{(2)}\right)^{*} T_{\overline{\mathcal{M}}_{0,2}(X, e)}$ is generated by global sections.

Denote by $\Sigma^{(2)}$ the surface containing $\bar{\Sigma}^{(2)}$ and $\Sigma$ obtained by identifying the divisors $\bar{\sigma}^{(2)}(B) \subset$ $\bar{\Sigma}^{(2)}$ and $\sigma(B) \subset \Sigma$ via $\bar{\sigma}^{(2)}(b) \leftrightarrow \sigma(b)$. Denote by $p^{(2)}: \Sigma^{(2)} \rightarrow B$ the unique morphism whose restriction to $\bar{\Sigma}^{(2)}$ is $\bar{p}^{(2)}$ and whose restriction to $\Sigma$ is $p$. Denote by $g^{(2)}: \Sigma^{(2)} \rightarrow X$ the unique morphism whose restriction to $\bar{\Sigma}^{(2)}$ is $\bar{g}^{(2)}$ and whose restriction to $\Sigma$ is $g$. Denote by $\sigma^{(2)}: B \rightarrow \Sigma^{(2)}$ the morphism obtained by composing $\varsigma^{(2)}$ with the inclusion $\bar{\Sigma}^{(2)} \subset \Sigma^{(2)}$. Then

$$
\zeta^{(2)}=\left(\left(p^{(2)}: \Sigma^{(2)} \rightarrow B, \sigma^{(2)}\right), g^{(2)}: \Sigma^{(2)} \rightarrow X\right)
$$

is a family of stable maps in the boundary $\Delta_{e} \subset \overline{\mathcal{M}}_{0,1}(X, e+1)$. Denote by $\tau^{(2)}$ the stable $A$-graph with two vertices $v$ and $\bar{v}$ of degree 1 and $e$ respectively, a single tail attached to $\bar{v}$, and a single edge connecting $v$ and $\bar{v}$. Then $\zeta^{(2)}$ factors through the canonical 1-morphism of Behrend-Manin stacks $\overline{\mathcal{M}}\left(X, \tau^{(2)}\right) \rightarrow \overline{\mathcal{M}}_{0,1}(X, e+1)$.

CLAim 5.16. The pullback $\left(\zeta^{(2)}\right)^{*} T_{\overline{\mathcal{M}}_{0,1}(X, e)}$ is generated by global sections.

By Lemma 3.28, there is a short exact sequence,

$$
0 \longrightarrow \zeta_{1}^{*} T_{\mathrm{ev}} \longrightarrow\left(\zeta^{(2)}\right)^{*} T_{\overline{\mathcal{M}}\left(X, \tau^{(2)}\right)} \longrightarrow\left(\bar{\zeta}_{e}^{(2)}\right)^{*} T_{\overline{\mathcal{M}}_{0,2}(X, e)} \longrightarrow 0 .
$$

By Claim 5.15, the third term is generated by global sections. Since $\zeta_{1}$ is very twisting, by Definition $4.3, \zeta_{1}^{*} T_{\mathrm{ev}}$ is ample. In particular it is generated by global sections. By Lemma 2.3, part iii, $\left(\zeta^{(2)}\right)^{*} T_{\overline{\mathcal{M}}\left(X, \tau^{(2)}\right)}$ is generated by global sections. By Lemma 3.29 there is a short exact sequence,

$$
0 \rightarrow\left(\zeta^{(2)}\right)^{*} T_{\overline{\mathcal{M}}\left(X, \tau^{(2)}\right)} \rightarrow\left(\zeta^{(2)}\right)^{*} T_{\overline{\mathcal{M}}_{0,1}(X, e+1)} \rightarrow \sigma^{*} \mathcal{O}_{\Sigma}(\sigma(B)) \otimes_{\mathcal{O}_{B}}\left(\bar{\sigma}^{(2)}\right)^{*} \mathcal{O}_{\bar{\Sigma}^{(2)}}\left(\bar{\sigma}^{(2)}(B)\right) \rightarrow 0 .
$$

By construction, $\mathcal{O}_{\bar{\Sigma}^{(2)}}\left(\bar{\sigma}^{(2)}(B)\right)$ is isomorphic to $\mathcal{O}_{B}$. Since $\zeta_{1}$ is very twisting, by Definition 4.3, $\sigma^{*} \mathcal{O}_{\Sigma}(\sigma(B))$ is generated by global sections. Hence the third term in the short exact sequence is generated by global sections. Since also the first term is generated by global sections, by Lemma 2.3, part iii, $\left(\zeta^{(2)}\right)^{*} T_{\overline{\mathcal{M}}_{0,1}(X, e+1)}$ is generated by global sections.

If $e>1$, the image of $\zeta^{(2)}$ intersects the divisor $\Delta_{1}$ transversely at the images of the points $q_{1}, \ldots, q_{\bar{\delta}}$. In particular, the degree of the $\mathbb{Q}$-Cartier divisor class $\left(\zeta^{(2)}\right)^{*} \mathcal{O}_{\overline{\mathcal{M}}_{0,1}(X, e+1)}\left(\Delta_{1}\right)$ is $\bar{\delta}$, which is positive. If $e=1$, then $\Delta_{1}=\Delta_{e}$. In this case $\overline{\mathcal{M}}\left(X, \tau^{(2)}\right)$ is the normalization of $\Delta_{1,1}$ in a neighborhood of $\zeta^{(2)}(B)$. So the degree of $\left(\zeta^{(2)}\right)^{*} \mathcal{O}_{\overline{\mathcal{M}}_{0,1}(X, 2)}\left(\Delta_{1}\right)$ is the sum of the degree of the pullback of the normal sheaf of $\overline{\mathcal{M}}\left(X, \tau^{(2)}\right) \rightarrow \overline{\mathcal{M}}_{0,1}(X, 2)$ and the degree of the divisor $q_{1}+\cdots+q_{\bar{\delta}}$, i.e. $\delta+\bar{\delta}$. So also in this case the degree of $\left(\zeta^{(2)}\right)^{*} \mathcal{O}_{\overline{\mathcal{M}}_{0,1}(X, e+1)}\left(\Delta_{1}\right)$ is positive.

Similar computations give that $\operatorname{deg}\left(\left(\zeta^{(2)}\right)^{*} \Delta_{i}\right)=\bar{\zeta}_{e}^{*} \Delta_{i-1}$ for $i=2, \ldots, e-1$. The curve $\zeta^{(2)}(B)$ is contained in the divisor $\Delta_{e}$, which is the image of $\overline{\mathcal{M}}\left(X, \tau^{(2)}\right)$. As noted above, by Lemma 3.29, the morphism $\overline{\mathcal{M}}\left(X, \tau^{(2)}\right) \rightarrow \overline{\mathcal{M}}_{0,1}(X, e+1)$ is unramified and the pullback by $\zeta^{(2)}$ of the normal sheaf has degree $\operatorname{deg}\left(\sigma^{*} \mathcal{O}_{\Sigma}(\sigma(B))\right)=\delta$. If $e>1$, then the image of $\zeta^{(2)}(B)$ is contained in the smooth locus of $\Delta_{e}$ so that the degree of $\left(\zeta^{(2)}\right)^{*} \mathcal{O}_{\overline{\mathcal{M}}_{0,1}(X, e+1)}\left(\Delta_{e}\right)$ is precisely $\delta$. If $e=1$, then each point $q_{1}, \ldots, q_{\bar{\delta}}$ maps to a point of $\Delta_{1}$ where $\Delta_{1}$ intersects itself transversely. So if $e=1$, the total degree of $\left(\zeta^{(2)}\right)^{*} \mathcal{O}_{\overline{\mathcal{M}}_{0,1}(X, 2)}\left(\Delta_{1}\right)$ is $\delta+\bar{\delta}$ (this is the same result from the last paragraph; it is included for the sake of consistency).

It is evident that

$$
\operatorname{deg}\left(\left(\zeta^{(2)}\right)^{*} \mathcal{H}\right)=\operatorname{deg}\left(\bar{\zeta}_{e}^{*} \mathcal{H}\right)+\operatorname{deg}\left(\zeta_{1}^{*} \mathcal{H}\right)
$$




\section{RATIONAL CURVES ON HYPERSURFACES, II}

and $\operatorname{deg}\left(\left(\zeta^{(2)}\right)^{*} \mathcal{L}\right)=\operatorname{deg}\left(\bar{\zeta}_{e}^{*} \mathcal{L}\right)$. As a 'consistency check' for the computations of these degrees, observe that, by [Pan99, Lemma 2.2.2],

$$
\operatorname{deg}\left(\sigma^{(2)}\right)^{*} \mathcal{O}_{\Sigma^{(2)}}\left(\sigma^{(2)}(B)\right)=\operatorname{deg}\left(\left(\zeta^{(2)}\right)^{*}\left(\frac{2}{e+1} \mathcal{L}-\frac{1}{(e+1)^{2}} \mathcal{H}-\sum_{i=1}^{e} \frac{(e+1-i)^{2}}{(e+1)^{2}} \Delta_{i}\right)\right) .
$$

Substituting the values above, using the formulas for $\delta$ and $\bar{\delta}$, and using that $\zeta_{1}^{*} \mathcal{L}=\bar{\zeta}_{e+1}^{*} \mathcal{L}$, the degree of the right-hand side is 0 . Of course this is correct; $\sigma^{(2)}(B) \subset \Sigma^{(2)}$ has self-intersection 0 by construction.

The family $\bar{\xi}_{0, e+1}$. Before proceeding to the construction of the families $\bar{\xi}_{d, e+1}$, the family $\zeta^{(2)}$ is deformed to a typical family $\bar{\xi}_{0, e+1}$; cf. Definition 5.8. The families $\bar{\xi}_{d, e+1}$ will be constructed as suitable modifications of $\bar{\xi}_{0, e+1}$.

Since $\zeta^{(2)}: B \rightarrow \overline{\mathcal{M}}_{0,1}(X, e+1)$ is free, i.e. $\left(\zeta^{(2)}\right)^{*} T_{\overline{\mathcal{M}}_{0,1}(X, e+1)}$ is generated by global sections, it follows by the same argument as in the proof of Lemma 4.14, part ii and [Kol96, Proposition 3.7] that there exists a deformation $\overline{x i}_{0, e+1}: B \rightarrow \overline{\mathcal{M}}_{0,1}(X, e+1)$ of $\widetilde{\zeta}$ that is in general position. Here deformation means that both $\zeta^{(2)}$ and $\bar{\xi}_{0, e+1}$ have a common generization. Moreover, for any finite collection of open conditions that are satisfied by $\zeta^{(2)}$, the deformation $\bar{\xi}_{0, e+1}$ may be chosen also to satisfy these conditions. Denote the family of stable maps by

$$
\bar{\xi}_{0, e+1}=\left(\left(\bar{p}_{0, e+1}: \bar{\Sigma}_{0, e+1} \rightarrow B, \bar{\sigma}_{0, e+1}\right), \bar{g}_{0, e+1}: \bar{\Sigma}_{0, e+1} \rightarrow X\right) .
$$

Denote by $\bar{h}_{0, e+1}: B \rightarrow X$ the composition $\bar{g}_{0, e+1} \circ \bar{\sigma}_{0, e+1}$.

Claim 5.17. There exists a deformation $\bar{\xi}_{0, e+1}$ of $\zeta^{(2)}$ such that:

i) $\bar{\xi}_{0, e+1}$ is typical,

ii) $\left(\operatorname{pr} \circ \bar{\xi}_{0, e+1}\right) * T_{\overline{\mathcal{M}}_{0,0}(X, e+1)}$ is ample,

iii) $\bar{h}_{0, e+1}: B \rightarrow X$ is very twistable,

iv) and $\left(\bar{\sigma}_{0, e+1}\right) * \mathcal{O}_{\bar{\Sigma}_{0, e+1}}\left(\bar{\sigma}_{0, e+1}(B)\right)$ is isomorphic to $\mathcal{O}_{B}$.

It suffices to prove that, for a general deformation $\bar{\xi}_{0, e+1}$ of $\zeta^{(2)}, \bar{\xi}_{0, e+1}$ satisfies the properties in Claim 5.17, i.e. for every irreducible component of the $\operatorname{Hom} \operatorname{stack} \operatorname{Hom}\left(B, \overline{\mathcal{M}}_{0,1}(X, e)\right)$ that contains $\zeta^{(2)}$, each of the conditions i-iv holds on a dense open substack.

i) By Lemmas 5.9 and 5.10, a general deformation of $\zeta^{(2)}$ is typical.

ii) The pullback by $\operatorname{pr} \circ \zeta^{(2)}: B \rightarrow \overline{\mathcal{M}}_{0,0}(X, e+1)$ of $T_{\overline{\mathcal{M}}_{0,0}(X, e+1)}$ equals $\left(\zeta^{(1)}\right)^{*} T_{\overline{\mathcal{M}}_{0,0}(X, e)}$. By Claim 5.14, this is ample. The condition on deformations of $\zeta^{(2)}$ that the pullback of $T_{\overline{\mathcal{M}}_{0,0}(X, e)}$ is ample is an open condition by Lemmas 2.8 and 2.9 (or more direct arguments). Therefore the pullback $\left(\operatorname{pr} \circ \bar{\xi}_{0, e+1}\right) * T_{\overline{\mathcal{M}}_{0,0}(X, e)}$ is ample.

iii) The morphism $g^{(2)} \circ \sigma^{(2)}: B \rightarrow X$ equals $\bar{g} \circ \varsigma$, and this is a deformation of $\bar{g} \circ \bar{\sigma}=g \circ \sigma$. Because $\zeta_{1}$ is very twisting, $g \circ \sigma$ is very twistable. By Proposition 4.8, $\varsigma$ can be chosen so that $g^{(2)} \circ \sigma^{(2)}$ is very twistable. Since $\bar{h}_{0, e+1}$ is a deformation of $g^{(2)} \circ \sigma^{(2)}$, by Proposition $4.8, \bar{\xi}_{0, e+1}$ can be chosen so that $\bar{h}_{0, e+1}$ is very twistable.

iv) Since $\left(\sigma^{(2)}\right)^{*} \mathcal{O}_{\Sigma^{(2)}}\left(\sigma^{(2)}(B)\right)$ is isomorphic to $\mathcal{O}_{B}$, also $\left(\bar{\sigma}_{0, e+1}\right)^{*} \mathcal{O}_{\bar{\Sigma}_{0, e+1}}\left(\bar{\sigma}_{0, e+1}(B)\right)$ is isomorphic to $\mathcal{O}_{B}$. This proves Claim 5.17. In particular, $\bar{\xi}_{0, e+1}$ is positive and $\bar{\xi}_{0, e+1}(B)$ is contained in the locus of very stable maps. Unfortunately it is not very positive!

To be a bit more precise in the proof of condition iii, there exists a deformation of $\zeta_{1}, \xi_{0,1}: B \rightarrow$ $\overline{\mathcal{M}}_{0,1}(X, 1)$ that is very twisting and such that $h_{0,1}:=g_{0,1} \circ \sigma_{0,1}: B \rightarrow X$ equals $\bar{h}_{0, e+1}$. 


\section{J. HARRIS AND J. STARR}

Because $\bar{\xi}_{0, e+1}$ is a deformation of $\zeta^{(2)}$, the intersection number of $\bar{\xi}_{0, e+1}(B)$ with any divisor of $\overline{\mathcal{M}}_{0,1}(X, e+1)$ is equal to the intersection number of $\zeta^{(2)}(B)$ with that divisor. These intersection numbers were computed above. Similarly, the intersection number of $\xi_{0,1}(B)$ with any divisor in $\overline{\mathcal{M}}_{0,1}(X, 1)$ is equal to the intersection number of $\zeta_{1}(B)$ with that divisor.

The families $\bar{\xi}_{d, e+1}$. The families $\bar{\xi}_{d, e+1}$ and $\xi_{d, 1}$ are constructed by induction on $d$. The base case $d=0$ is the pair $\left(\xi_{0,1}, \bar{\xi}_{0, e+1}\right)$ constructed above, and the induction step consists of performing a single modification and then deforming.

Let $d$ be an integer $1 \leqslant d \leqslant \bar{\delta}$. By way of induction, suppose that a pair $\left(\xi_{d-1,1}, \bar{\xi}_{d-1, e+1}\right)$ has been constructed such that $\xi_{d-1,1}: B \rightarrow \overline{\mathcal{M}}_{0,1}(X, 1)$ is very twisting, $\bar{\xi}_{d-1, e+1}: B \rightarrow \overline{\mathcal{M}}_{0,1}(X, e+1)$ is very positive and typical (in particular $\bar{\xi}_{d-1, e+1}(B)$ is contained in the locus of very stable maps), the map $h_{d-1,1}: B \rightarrow X$ equals the map $\bar{h}_{d-1, e+1}: B \rightarrow X$, and the degree conditions of Equation (9) hold for $d-1$. In particular, because $d \leqslant \bar{\delta}, \operatorname{deg}\left(\bar{\xi}_{d-1, e+1}^{*} \Delta_{1}\right) \geqslant 1$.

Let $b \in B$ be a closed point such that the stable map $\left(\bar{\xi}_{d-1, e+1}\right)_{b}$ is in $\Delta_{1}$. By Definition 5.8, this stable map is of the form,

$$
\left(\bar{\xi}_{d-1, e+1}\right)_{b}=\left(\left(L \cup M, \bar{\sigma}_{d-1, e+1}(b)\right),\left(\bar{g}_{d-1, e+1}\right)_{b}: L \cup M \rightarrow X\right),
$$

where $\bar{\sigma}_{d-1, e+1}(b) \in L$ and $\left.g\right|_{L}: L \rightarrow X$ is an isomorphism to a twistable line.

Denote by $\widetilde{B}$ the prestable curve of genus 0 containing $B$ and $L$ obtained by identifying the divisor $b \in B$ with the divisor $\bar{\sigma}_{d-1, e+1}(b) \in L$. Denote by

$$
\widetilde{\xi}_{d-1, e+1}=\left(\left(\widetilde{p}_{d-1, e+1}: \widetilde{\Sigma}_{d-1, e+1} \rightarrow \widetilde{B}, \widetilde{\sigma}_{d-1, e+1}\right), \widetilde{g}_{d-1, e+1}: \widetilde{B} \rightarrow X\right)
$$

the modification of $\bar{\xi}_{d-1, e+1}$ associated to $\left(b, L, \bar{\sigma}_{d-1, e+1}(b)\right)$; cf. Definition 5.3.

By construction, for each divisor in $\overline{\mathcal{M}}_{0,1}(X, e+1)$, the difference of the intersection number with $\widetilde{\xi}_{d-1, e+1}(\widetilde{B})$ and the intersection number with $\bar{\xi}_{d-1, e+1}(B)$ equals the intersection number with $\widetilde{\xi}_{d-1, e+1}(L)$, i.e.

$$
\begin{cases}\widetilde{\xi}_{d-1, e+1}^{*} \mathcal{H}=\bar{\xi}_{d-1, e+1}^{*} \mathcal{H}, & \\ \widetilde{\xi}_{d-1, e+1}^{*} \mathcal{L}=\bar{\xi}_{d-1, e+1}^{*} \mathcal{L}+1, & \\ \widetilde{\xi}_{d-1, e+1}^{*} \Delta_{i}=\bar{\xi}_{d-1, e+1}^{*} \Delta_{i}, & i=2, \ldots, e-1, \\ \widetilde{\xi}_{d-1, e+1}^{*} \Delta_{1}=\bar{\xi}_{d-1, e+1}^{*} \Delta_{1}-1, & i>1, \\ \widetilde{\xi}_{d-1, e+1}^{*} \Delta_{e}=\bar{\xi}_{d-1, e+1}^{*} \Delta_{e}+1, & i>1, \\ \widetilde{\xi}_{d-1,2}^{*} \Delta_{1}=\bar{\xi}_{d-1, e+1}^{*} \Delta_{1}, & i=1 .\end{cases}
$$

Just a few remarks should be made about this list. The second line arises because $\widetilde{h}_{d-1, e+1}: L \rightarrow X$ equals $\left.g\right|_{L}: L \rightarrow X$, which is an isomorphism to a line. The fourth line arises by applying Lemma 3.29 and using the fact that the pullback of $\mathcal{O}_{\overline{\mathcal{M}}_{0,1}(X, e+1)}\left(\Delta_{1}\right)$ equals

$$
\sigma_{I, \lambda}^{*} \mathcal{O}_{\Lambda}\left(\sigma_{I, \lambda}(L)\right) \otimes\left(\sigma_{I, \lambda}^{\prime}\right)^{*} \mathcal{O}_{L \times M}\left(\sigma_{I, \lambda}^{\prime}(L)\right),
$$

in the notation of $\S 5.1$, where $\lambda$ is the point in $L \cap M$. Of course the second factor in the tensor product is isomorphic to $\mathcal{O}_{L}$ and the first factor is $\mathcal{O}_{L}(-\lambda)$ (because of the blowing-up of $L \times L$ at $(\lambda, \lambda)$ ); hence the net degree change is -1 . Similarly, the fifth line arises because the intersection of $\widetilde{\xi}_{d-1, e+1}(L)$ and $\Delta_{e}$ is exactly $\widetilde{\xi}_{d-1, e+1}(\lambda)$; hence the degree increases by 1 . In the case $e=1$, the last two contributions exactly cancel each other, which gives the last line.

As a 'consistency check', substituting the computations above into the formula for the selfintersection of $\widetilde{\sigma}_{d-1, e+1}(\widetilde{B}) \subset \widetilde{\Sigma}_{d-1, e+1}$ [Pan99, Lemma 2.2.2] yields a net change of +1 . Of course 


\section{RATIONAL CURVES ON HYPERSURFACES, II}

this is correct because the change equals the self-intersection of $\sigma_{I, \Delta}(L) \subset \Lambda$, which is $T_{L}(-\lambda)$ (because of the blowing-up of $L \times L$ at $(\lambda, \lambda)$ ), and this has degree $2-1=1$.

Claim 5.18. The 1-morphism $\widetilde{\xi}_{d-1, e+1}: \widetilde{B} \rightarrow \overline{\mathcal{M}}_{0,1}(X, e+1)$ is very positive, the image is contained in the locus of stable maps with only the trivial automorphism, and the hypotheses of Lemma 5.6 are satisfied.

First of all, the restriction of $\widetilde{h}_{d-1, e+1}: \widetilde{B} \rightarrow X$ to $B$ is $\bar{h}_{d-1, e+1}$ and the restriction to $L$ is $\left.g\right|_{L}: L \rightarrow X$. By hypothesis, $\bar{h}_{d-1, e+1}$ is stable, and $\left.g\right|_{L}$ is stable because it is a closed immersion. Therefore $\bar{h}_{d-1, e+1}$ is stable. By hypothesis, the dimension of the obstruction group of $\bar{h}_{d-1, e+1}$ is 0 . Moreover, possibly after deforming $\bar{\xi}_{d-1, e+1}$, it may be assumed that the node of $L \cap M$ maps to a very general point of $X$. Therefore $\left.g\right|_{L}: L \rightarrow X$ is free by [Deb01, Proposition 4.14]. So the dimension of the obstruction group of $\left.g\right|_{L}$ is 0 . Also, by Hypothesis 4.10 it may be assumed that ev $: \overline{\mathcal{M}}_{0,1}(X, 1) \rightarrow X$ is smooth at $\left(\left(L, \bar{\sigma}_{d-1, e+1}(b)\right),\left.g\right|_{L}: L \rightarrow X\right)$. So by Lemma 3.28, the dimension of the obstruction group of $\widetilde{h}_{d-1, e+1}$ is 0 . Hence parts i and ii of Definition 4.11 are satisfied.

By hypothesis, the dimension of the obstruction group of $\overline{\mathcal{M}}_{0,0}(X, e+1)$ at each point of $\operatorname{pr}\left(\widetilde{\xi}_{d-1, e+1}(B)\right)=\operatorname{pr}\left(\bar{\xi}_{d-1, e+1}(B)\right)$ is $0 . \operatorname{pr}\left(\widetilde{\xi}_{d-1, e+1}(L)\right)$ is the point $\operatorname{pr}\left(\left(\widetilde{\xi}_{d-1, e+1}\right)_{b}\right)$, which is one of the points above. Thus part iii of Definition 4.11 is satisfied. Similarly, the restriction to $B$ of the pullback

$$
\left(\operatorname{pr} \circ \widetilde{\xi}_{d-1, e+1}\right)^{*} T_{\overline{\mathcal{M}}_{0,0}(X, e+1)}
$$

equals the pullback associated to $\operatorname{pr} \circ \bar{\xi}_{d-1, e+1}$, which is deformation ample by hypothesis. The restriction to $L$ is the pullback of a vector bundle by a constant map, and hence it is isomorphic to a direct sum of copies of $\mathcal{O}_{L}$. In particular the restriction to $L$ is generated by global sections. By Lemma 2.11, the pullback is deformation ample on all of $\widetilde{B}$; hence part iv of Definition 4.11 is satisfied.

Finally, the restriction to $B$ of the pullback

$$
\tilde{\sigma}_{d-1, e+1}^{*} \mathcal{O}_{\widetilde{\Sigma}_{d-1, e+1}}\left(\widetilde{\sigma}_{d-1, e+1}(\widetilde{B})\right)
$$

is equal to the analogous sheaf for $\bar{\xi}_{d-1, e+1}$, and this is generated by global sections by construction. The restriction to $L$ is the pullback by the diagonal of $T_{L}$, twisted down by $\bar{\sigma}_{d-1, e+1}(b)$, i.e. $\mathcal{O}_{L}(2-1)=\mathcal{O}_{L}(1)$. Thus by Lemma 2.11 , the restriction to all of $\widetilde{B}$ is deformation ample. Hence part v of Definition 4.11 is satisfied; therefore $\widetilde{\xi}_{d-1, e+1}$ is very positive.

By hypothesis $\widetilde{\xi}_{d-1, e+1}(B)=\bar{\xi}_{d-1, e+1}(B)$ is contained in the locus of stable maps that have only the trivial automorphism. In particular, $\left(\bar{\xi}_{d-1, e+1}\right)_{b}$ has only the trivial automorphism, from which it easily follows that $\widetilde{\xi}_{d-1, e+1}(L)$ is contained in the locus of stable maps with only the trivial automorphism. Therefore the image of all of $\widetilde{B}$ is contained in the locus of stable maps with only the trivial automorphism.

Because $\bar{\xi}_{d-1, e+1}: B \rightarrow \overline{\mathcal{M}}_{0,1}(X, e+1)$ is positive and the image is contained in the locus of very stable curves, the hypotheses of Lemma 3.22 are satisfied and there is a short exact sequence of locally free $\mathcal{O}_{B}$-modules,

$$
\begin{gathered}
0 \rightarrow \bar{\sigma}_{d-1, e+1}^{*} \mathcal{O}_{\bar{\Sigma}_{d-1, e+1}}\left(\bar{\sigma}_{d-1, e+1}(B)\right) \rightarrow \cdots \\
\bar{\xi}_{d-1, e+1}^{*} T_{\overline{\mathcal{M}}_{0,1}(X, e+1)} \rightarrow\left(\operatorname{pr} \circ \bar{\xi}_{d-1, e+1}\right)^{*} T_{\overline{\mathcal{M}}_{0,0}(X, e+1)} \rightarrow 0 .
\end{gathered}
$$

By hypothesis, the third term is deformation ample, and the first term is generated by global sections. By Lemma 2.3, part iii, the pullback of $T_{\overline{\mathcal{M}}_{0,1}(X, e+1)}$ is generated by global sections. Because the node of $L \cap M$ is mapped to a very general point of $X$, by [Deb01, Proposition 4.14], 


\section{J. HARRIS AND J. STARR}

the pullback of $T_{X}$ to $L$ and the pullback to $M$ are each generated by global sections; in particular the dimension of the obstruction group of $\left.g\right|_{L}: L \rightarrow X$ is 0 . Hence the hypotheses of Lemma 5.6 are satisfied; therefore Claim 5.18 is true.

Of course the next step will be to apply Lemma 5.6 to construct $\bar{\xi}_{d, e+1}$. But first the family $\widetilde{\xi}_{d-1,1}$ is constructed. By hypothesis, the line $L$ is twistable. Denote by

$$
\xi_{L, 1}=\left(\left(p_{L, 1}: \Sigma_{L, 1} \rightarrow L, \sigma_{L, 1}\right), g_{L, 1}: \Sigma_{L, 1} \rightarrow X\right)
$$

a twisting family such that $h_{L, 1}=\left.g\right|_{L}$. If $X$ is of planar type, assume that the degree of $g_{L, 1}$ is 1 ; otherwise the degree of $g_{L, 1}$ is 2 . By Hypotheses 1.6 and 4.10, Lemma 4.9 applies to $\xi_{d-1,1}$ and $\xi_{L, 1}$, i.e. possibly after deforming the two families (without deforming $\widetilde{h}_{d-1, e+1}$ ),

$$
\left(\xi_{d-1,1}\right)_{b}=\left(\xi_{L, 1}\right)_{\bar{\sigma}_{d-1, e+1}(b)}
$$

Define $\widetilde{\xi}_{d-1,1}: \widetilde{B} \rightarrow \overline{\mathcal{M}}_{0,1}(X, 1)$ to be the unique 1-morphism whose restriction to $B$ is $\xi_{d-1,1}$ and whose restriction to $L$ is $\xi_{L, 1}$. By Lemma $4.5, \widetilde{\xi}_{d-1,1}$ is very twisting. By construction, $\widetilde{h}_{d-1,1}=\widetilde{h}_{d-1, e+1}$. Also,

$$
\operatorname{deg}\left(\widetilde{\xi}_{d-1,1}^{*} \mathcal{H}\right)=\operatorname{deg}\left(\xi_{d-1,1}^{*} \mathcal{H}\right)+\operatorname{deg}\left(g_{L, 1}\right) .
$$

By definition, $\operatorname{deg}\left(g_{L, 1}\right)$ is 1 if $X$ is planar type, and 2 if $X$ is quadric type. Finally,

$$
\operatorname{deg}\left(\widetilde{\xi}_{d-1,1}^{*} \mathcal{L}\right)=\operatorname{deg}\left(\xi_{d-1,1}^{*} \mathcal{L}\right)+1
$$

because $h_{L, 1}: L \rightarrow X$ is an isomorphism to a line.

By Lemma 5.6, there exists a 1-morphism $\bar{\xi}_{d, e+1}: B \rightarrow \overline{\mathcal{M}}_{0,1}(X, e+1)$, i.e. both $\widetilde{\xi}_{d-1, e+1}$ and $\bar{\xi}_{d, e+1}$ have a common generization. Since the image of $\widetilde{\xi}_{d-1, e+1}$ is contained in the locus of stable maps with only the trivial automorphism, $\bar{\xi}_{d, e+1}$ can be chosen with the same property. Because the dimension of the obstruction group at every point of the image of $\widetilde{\xi}_{d-1, e+1}$ equals 0 , the same is true for $\bar{\xi}_{d, e+1}$. Hence the image of $\bar{\xi}_{d, e+1}$ is contained in the locus of $\overline{\mathcal{M}}_{0,1}(X, e+1)$ that is a smooth scheme. Because $\widetilde{\xi}_{d-1, e+1}$ can be chosen to contain a very general point of $\overline{\mathcal{M}}_{0,1}(X, e+1)$ (because $\bar{\xi}_{d-1, e+1}$ is typical), the same is true of $\bar{\xi}_{d, e+1}$. By [Deb01, Proposition 4.14], the pullback $\bar{\xi}_{d, e+1}^{*} T_{\overline{\mathcal{M}}_{0,1}(X, e+1)}$ is generated by global sections. Therefore, after deforming further, $\bar{\xi}_{d, e+1}$ can be chosen to be typical. Because $\widetilde{\xi}_{d-1, e+1}$ is very positive, by Lemma $4.13, \bar{\xi}_{d, e+1}$ can be chosen to be very positive. Also, by the proof of Proposition $4.8, \bar{\xi}_{d, e+1}$ can be chosen so that there exists a deformation $\xi_{d, 1}$ of $\widetilde{\xi}_{d-1,1}$ that is very twisting and such that $h_{d, 1}=\bar{h}_{d, e+1}$.

Of course for any divisor in $\overline{\mathcal{M}}_{0,1}(X, e+1)$ (respectively $\overline{\mathcal{M}}_{0,1}(X, 1)$ ), the intersection number

with $\bar{\xi}_{d, e+1}$ (respectively $\xi_{d, 1}$ ) equals the intersection number with $\widetilde{\xi}_{d-1, e+1}$ (respectively $\widetilde{\xi}_{d-1,1}$ ), and these are computed above. This finishes the proof of the induction step. Hence, by induction on $d$, for each $d=1, \ldots, \bar{\delta}$, there exists an inducting pair $\left(\xi_{d, 1}, \bar{\xi}_{d, e+1}\right)$ as claimed. This completes the proof of Theorem 5.13.

\section{Twistable lines on hypersurfaces}

In this section it is proved that if $n+1 \geqslant d^{2}$, if $X \subset \mathbb{P}^{n}$ is a general hypersurface of degree $d$, and if $L \subset X$ is a general line on $X$, then $L$ is twistable.

Remark 6.1. There is one exceptional case, namely $d=1$. For $n=1,2$, there is no twistable line on a hyperplane in $\mathbb{P}^{n}$, i.e. there is no twistable line on $\mathbb{P}^{n-1}$. For $n \geqslant 3$ there is a twistable line on $\mathbb{P}^{n-1}$. In this section it is proved there is a twistable line on $\mathbb{P}^{n-1}$ if $n \geqslant 4$. Let $((\pi: \Sigma \rightarrow B, \sigma), g)$ be a twisting family in $\mathbb{P}^{n}$ such that $h: B \rightarrow \mathbb{P}^{n}$ is a line, and let $p \in \mathbb{P}^{n}-g(\Sigma)$. Denote by 


\section{RATIONAL CURVES ON HYPERSURFACES, II}

$\operatorname{proj}_{p}: \mathbb{P}^{n} \rightarrow \mathbb{P}^{n-1}$ projection from $p$. Then $\left((\pi: \Sigma \rightarrow B, \sigma), \operatorname{proj}_{p} \circ g\right)$ is a twisting family in $\mathbb{P}^{n-1}$ and $\operatorname{proj}_{p} \circ h: B \rightarrow \mathbb{P}^{n-1}$ is a line. Therefore there is also a twistable line on $\mathbb{P}^{2}$.

Notation 6.2. Denote $N_{d}=\left(\begin{array}{c}n+d \\ n\end{array}\right)-1$ and denote by $\mathbb{P}^{N_{d}}$ the projective space parametrizing hypersurfaces $X \subset \mathbb{P}^{n}$ of degree $d$. Denote by $\mathcal{X} \subset \mathbb{P}^{N_{d}} \times \mathbb{P}^{n}$ the universal family of degree- $d$ hypersurfaces in $\mathbb{P}^{n}$. Denote by $\mathbb{G}(1, n)$ the Grassmannian variety of lines in $\mathbb{P}^{n}$. Denote by $F(\mathcal{X}) \subset \mathbb{P}^{N_{d}} \times \mathbb{G}(1, n)$ the parameter space of pairs $([X],[L])$ consisting of a hypersurface of degree $d, X \subset \mathbb{P}^{n}$, and a line $L \subset X$. Denote by $P(t)=(t+1)^{2}$ the Hilbert polynomial of a quadric surface in $\mathbb{P}^{3}$. Denote by $U \subset \operatorname{Hilb}_{\mathbb{P} n}^{P(t)}$ the open subscheme of the Hilbert scheme that parametrizes subschemes of $\mathbb{P}^{n}$ projectively equivalent to a smooth quadric surface in $\mathbb{P}^{3} \subset \mathbb{P}^{n}$. Denote by $V \subset U \times \mathbb{G}(1, n)$ the parameter space of pairs $([\Sigma],[L])$ consisting of a smooth quadric surface, $\Sigma$, and a line $L \subset \Sigma$. Denote by $V \rightarrow \tilde{U} \rightarrow U$ the Stein factorization of the projection $\mathrm{pr}_{U}: V \rightarrow U$. Denote by $W \subset \mathbb{P}^{N_{d}} \times U \times \mathbb{G}(1, n)$ the parameter space of triples $([X],[\Sigma],[L])$ consisting of a hypersurface of degree $d, X \subset \mathbb{P}^{n}$, a smooth quadric surface $\Sigma \subset X$, and a line $L \subset \Sigma$.

Observe that the projection $F(\mathcal{X}) \rightarrow \mathbb{G}(1, d)$ is a projective bundle of relative dimension $N_{d}-(d+1)$. Observe that $\tilde{U} \rightarrow U$ is a finite, étale morphism of degree 2 . Observe that $V \rightarrow \tilde{U}$ is a $\mathbb{P}^{1}$-bundle. Observe that $W \rightarrow V$ is a projective space bundle of relative dimension $N_{d}-(d+1)^{2}$.

Let $([X],[\Sigma],[L])$ be a triple in $W$. There is a map (well defined up to nonzero scalar) $\partial_{X}$ : $\mathbb{C}^{n+1} \rightarrow H^{0}\left(\mathbb{P}^{n}, \mathcal{O}_{\mathbb{P}^{n}}(d-1)\right)$ that evaluates the partial derivatives of a defining equation of $X$. Compose this map with the restriction map $H^{0}\left(\mathbb{P}^{n}, \mathcal{O}_{\mathbb{P}^{n}}(d-1)\right) \rightarrow H^{0}\left(\Sigma, \mathcal{O}_{\Sigma}(d-1)\right)$, and denote the composition by

$$
\partial_{X, \Sigma}: \mathbb{C}^{n+1} \longrightarrow H^{0}\left(\Sigma, \mathcal{O}_{\Sigma}(d-1)\right) .
$$

Denote $E=\mathcal{O}_{W}^{\oplus(n+1)}$. Denote by $G$ the unique quotient of $H^{0}\left(\mathbb{P}^{n}, \mathcal{O}_{\mathbb{P}^{n}}(d-1)\right) \otimes_{\mathbb{C}} \mathcal{O}_{U}$ that is locally free and whose fiber at each point $\Sigma$ is the quotient

$$
H^{0}\left(\mathbb{P}^{n}, \mathcal{O}_{\mathbb{P}^{n}}(d-1)\right) \longrightarrow H^{0}\left(\Sigma, \mathcal{O}_{\Sigma}(d-1)\right) .
$$

Denote by $F$ the locally free $\mathcal{O}_{W}$-module,

$$
F=\operatorname{pr}_{\mathbb{P}^{N_{d}}}^{*} \mathcal{O}_{\mathbb{P}^{N}}(1) \otimes \operatorname{pr}_{U}^{*} G .
$$

There is a map of $\mathcal{O}_{W}$-modules, $\partial: E \rightarrow F$ whose fiber at each point $([X],[\Sigma],[L])$ is the map $\partial_{X, \Sigma}$. Denote by $W^{\mathrm{o}} \subset W$ the open subscheme that is the complement of the support of $\operatorname{Coker}(\partial)$, i.e. $W^{\mathrm{o}}$ is the maximal open subscheme on which $\partial$ is surjective.

Lemma 6.3. Let $([X],[\Sigma],[L])$ be a point in $W^{\circ}$. Then,

i) $X$ is smooth along $\Sigma$,

ii) $h^{i}\left(\Sigma, N_{\Sigma / \mathbb{P}^{n}}\right)=h^{i}\left(\Sigma, N_{\Sigma / \mathbb{P}^{n}}(-L)\right)=h^{i}\left(\Sigma, N_{\Sigma / \mathbb{P}^{n}}(-1)\right)=0$, for $i>0$,

iii) $h^{i}\left(\Sigma, N_{\Sigma / X}\right)=h^{i}\left(\Sigma, N_{\Sigma / X}(-L)\right)=h^{i}\left(\Sigma, N_{\Sigma / X} \otimes \mathcal{O}_{\Sigma}(-1)\right)=0$, for $i>0$,

iv) $h^{1}\left(L, N_{L / X}(-1)\right)=h^{1}\left(L, N_{L / X}\right)=0$,

v) the projection morphism $\operatorname{pr}_{\mathbb{P}^{N}}: W \rightarrow \mathbb{P}^{N_{d}}$ is smooth at $([X],[\Sigma],[L])$,

vi) the projection morphism $\operatorname{pr}_{\mathbb{P}^{N_{d}}}: F(\mathcal{X}) \rightarrow \mathbb{P}^{N_{d}}$ is smooth at $([X],[L])$, and

vii) the projection morphism $\operatorname{pr}_{F(\mathcal{X})}: W \rightarrow F(\mathcal{X})$ is smooth at $([X],[\Sigma],[L])$.

Proof. i) Since the partial derivatives of a defining equation of $X$ generate $H^{0}\left(\Sigma, \mathcal{O}_{\Sigma}(d-1)\right)$, the subscheme of $X$ where the partial derivatives all vanish is disjoint from $\Sigma$. By the Jacobian criterion, $X$ is smooth at each point of $\Sigma$. 


\section{J. HARRIS AND J. STARR}

ii) Denote by $\mathbb{P}^{3} \subset \mathbb{P}^{n}$ the span of $\Sigma$. There is a short exact sequence,

$$
\left.0 \longrightarrow N_{\Sigma / \mathbb{P}^{3}} \longrightarrow N_{\Sigma / \mathbb{P}^{n}} \longrightarrow N_{\mathbb{P}^{3} / \mathbb{P}^{n}}\right|_{\Sigma} \longrightarrow 0 .
$$

Since $N_{\Sigma / \mathbb{P}^{3}} \cong \mathcal{O}_{\Sigma}(2)$ and since $\left.N_{\mathbb{P}^{3} / \mathbb{P}^{n}}\right|_{\Sigma} \cong \mathcal{O}_{\Sigma}(1)^{\oplus(n-3)}$, the short exact sequence above is

$$
0 \longrightarrow \mathcal{O}_{\Sigma}(2) \longrightarrow N_{\Sigma / \mathbb{P} n} \longrightarrow \mathcal{O}_{\Sigma}(1)^{\oplus(n-3)} \longrightarrow 0 .
$$

From this it is easy to compute that

$$
h^{i}\left(\Sigma, N_{\Sigma / \mathbb{P}^{n}}\right)=h^{i}\left(\Sigma, N_{\Sigma / \mathbb{P}^{n}} \otimes \mathcal{O}_{\Sigma}(-1)\right)=h^{i}\left(\Sigma, N_{\Sigma / \mathbb{P}^{n}} \otimes \mathcal{O}_{\Sigma}(-L)\right)=0,
$$

for $i>0$.

iii) There is a short exact sequence,

$$
\left.0 \longrightarrow N_{\Sigma / X} \longrightarrow N_{\Sigma / \mathbb{P}^{n}} \longrightarrow N_{X / \mathbb{P}^{n}}\right|_{\Sigma} \longrightarrow 0 \text {. }
$$

Of course $\left.N_{X / \mathbb{P}^{n}}\right|_{\Sigma} \cong \mathcal{O}_{\Sigma}(d)$. For the three cases $\mathcal{L}=\mathcal{O}_{\Sigma}, \mathcal{L}=\mathcal{O}_{\Sigma}(-L)$, and $\mathcal{L}=\mathcal{O}_{\Sigma}(-1)$, $h^{i}\left(\Sigma, \mathcal{O}_{\Sigma}(d) \otimes \mathcal{L}\right)=0$ for $i>0$. By part ii, $h^{i}\left(\Sigma, N_{\Sigma / \mathbb{P} n} \otimes \mathcal{L}\right)=0$ for $i>0$. By the long exact sequence in cohomology associated to the twist by $\mathcal{L}$ of the short exact sequence above, $h^{i}\left(\Sigma, N_{\Sigma / X} \otimes \mathcal{L}\right)=0$ for $i>2$. Also $h^{1}\left(\Sigma, N_{\Sigma / X} \otimes \mathcal{L}\right)=0$ if and only if the map $e_{\mathcal{L}}: H^{0}\left(\Sigma, N_{\Sigma / \mathbb{P} n} \otimes \mathcal{L}\right) \rightarrow H^{0}\left(\Sigma,\left.N_{X / \mathbb{P}^{n}}\right|_{\Sigma}\right)$ is surjective.

The map $e_{\mathcal{O}_{\Sigma}}$ factors the map $\partial_{X, \Sigma}$. Since $\partial_{X, \Sigma}$ is surjective, also $e_{\mathcal{O}_{\Sigma}}$ is surjective and $h^{1}\left(\Sigma, N_{\Sigma / X}\right)=0$.

For the case $\mathcal{L}=\mathcal{O}_{\Sigma}(-L)$, observe that $\mathcal{O}_{\Sigma}(1) \cong \mathcal{O}_{\Sigma}\left(L+L^{\prime}\right)$ where $L^{\prime} \subset \Sigma$ is a line of the ruling opposite to $L$. There is the following commutative diagram:

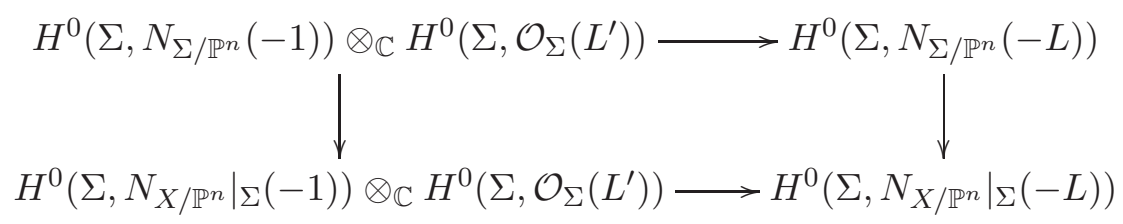

The left vertical arrow is surjective by part ii. The bottom arrow is

$$
H^{0}\left(\Sigma, \mathcal{O}_{\Sigma}\left((d-1) L+(d-1) L^{\prime}\right)\right) \otimes H^{0}\left(\Sigma, \mathcal{O}_{\Sigma}\left(L^{\prime}\right)\right) \longrightarrow H^{0}\left(\Sigma, \mathcal{O}_{\Sigma}\left((d-1) L+d L^{\prime}\right)\right),
$$

which is surjective. Therefore the right vertical arrow is also surjective, i.e. $h^{1}\left(\Sigma, N_{\Sigma / X}(-L)\right)=0$. The proof that $h^{1}\left(\Sigma, N_{\Sigma / X}\right)=0$ is almost identical to the proof that $h^{1}\left(\Sigma, N_{\Sigma / X}(-L)=0\right.$.

iv) There is a short exact sequence,

$$
\left.0 \longrightarrow N_{\Sigma / X}(-1) \longrightarrow N_{\Sigma / X}\left(-L^{\prime}\right) \longrightarrow N_{\Sigma / X}\right|_{L}(-1) \longrightarrow 0 .
$$

By the associated long exact sequence in cohomology and the computations above, $h^{1}\left(L,\left.N_{\Sigma / X}\right|_{L}(-1)\right)=0$. There is a short exact sequence,

$$
\left.0 \longrightarrow N_{L / \Sigma}(-1) \longrightarrow N_{L / X}(-1) \longrightarrow N_{\Sigma / X}\right|_{L}(-1) \longrightarrow 0 .
$$

Of course $N_{L / \Sigma} \cong \mathcal{O}_{L}(1)$, so $h^{1}\left(L, N_{L / \Sigma}(-1)\right)=0$. As proved above, $h^{1}\left(L,\left.N_{\Sigma / X}\right|_{L}(-1)\right)=0$. Therefore by the long exact sequence in cohomology, $h^{1}\left(L, N_{L / X}(-1)\right)=0$. Since $N_{L / X}$ is a locally free $\mathcal{O}_{L}$-module and $h^{1}\left(L, N_{L / X}(-1)\right)=0$, by Grothendieck's lemma $N_{L / X}$ is generated by global sections. In particular, also $h^{1}\left(L, N_{L / X}\right)=0$.

v) By [Kol96, Proposition I.2.14.2], the obstruction space for the relative Hilbert scheme, $\operatorname{Hilb}_{\mathcal{X} / \mathbb{P}^{N_{d}}}^{P(t)}$, at the point $([X],[\Sigma])$ is contained in $H^{1}\left(\Sigma, N_{\Sigma / X}\right)$, which has dimension 0 by part iv. Since the obstruction space vanishes, it follows by [Kol96, Theorem 2.10] that $\operatorname{Hilb}_{\mathcal{X} / \mathbb{P}^{N_{d}}}^{P(t)} \rightarrow \mathbb{P}^{N_{d}}$ is 


\section{RATIONAL CURVES ON HYPERSURFACES, II}

smooth at $([X],[\Sigma])$. Also $W \rightarrow \operatorname{Hilb}_{\mathcal{X} / \mathbb{P}^{N_{d}}}^{P(t)}$ is smooth. Therefore the composition $\operatorname{pr}_{\mathbb{P}^{N_{d}}}: W \rightarrow \mathbb{P}^{N_{d}}$ is smooth at $([X],[\Sigma],[L])$.

vi) The proof is almost identical to the proof of part $\mathrm{v}$.

vii) By part v, $W \rightarrow \mathbb{P}^{N_{d}}$ is smooth at $([X],[\Sigma],[L])$. By part vi, $F(\mathcal{X}) \rightarrow \mathbb{P}^{N_{d}}$ is smooth at $([X],[L])$. So the Jacobian criterion for the smoothness of $\operatorname{pr}_{F(\mathcal{X})}$ at $([X],[\Sigma],[L])$ is that the map of vertical tangent bundles, $d \operatorname{pr}_{F(\mathcal{X})}: T_{W / \mathbb{P}^{N_{d}}} \rightarrow \pi^{*} T_{F(\mathcal{X}) / \mathbb{P}^{N_{d}}}$ is surjective at $([X],[\Sigma],[L])$. This reduces to the surjectivity of $H^{0}\left(\Sigma, N_{\Sigma / X}\right) \rightarrow H^{0}\left(L,\left.N_{\Sigma / X}\right|_{L}\right)$. The cokernel is contained in $H^{1}\left(\Sigma, N_{\Sigma / X}(-L)\right)$, which is zero by part iii. Therefore $\operatorname{pr}_{F(\mathcal{X})}$ is smooth at $([X],[\Sigma],[L])$.

We associate to each $([X],[\Sigma],[L]) \in W^{\circ}$ a morphism $\zeta: L \rightarrow \overline{\mathcal{M}}_{0,1}(X, 1)$ as follows. Let $\sigma: L \rightarrow \Sigma$ be the inclusion and let $\operatorname{pr}_{L}: \Sigma \rightarrow L$ be the unique projection such that $\sigma$ is a section of $\operatorname{pr}_{L}$. Let $g: \Sigma \rightarrow X$ be the inclusion. Then,

$$
\zeta=\left(\left(\operatorname{pr}_{L}: \Sigma \rightarrow L, \sigma\right), g: \Sigma \rightarrow X\right)
$$

is a family of stable maps, i.e. a morphism $\zeta: L \rightarrow \overline{\mathcal{M}}_{0,1}(X, 1)$.

Lemma 6.4. For every point $([X],[\Sigma],[L]) \in W^{\mathrm{o}}$, the morphism $\zeta: L \rightarrow \overline{\mathcal{M}}_{0,1}(X, 1)$ is twisting.

Proof. Since $g \circ \sigma: L \rightarrow X$ is an embedding, axiom i of Definition 4.3 is satisfied. By Lemma 6.3, part vi, the dimension of the obstruction group of $\overline{\mathcal{M}}_{0,0}(X, 1)$ at $[g \circ \sigma: L \rightarrow X]$ is 0 , i.e. axiom ii of Definition 4.3 is satisfied.

Denote by $\mathcal{N}$ the normal bundle of the regular embedding $\left(\operatorname{pr}_{L}, g\right): \Sigma \rightarrow L \times X$. There is a short exact sequence,

$$
0 \longrightarrow \operatorname{pr}_{L}^{*} T_{L} \longrightarrow \mathcal{N} \longrightarrow N_{\Sigma / X} \longrightarrow 0 .
$$

By Remark 4.4, part ii, axiom iii holds if $R^{1}\left(\mathrm{pr}_{L}\right)_{*} \mathcal{N}(-\sigma)=\{0\}$. For each fiber $L^{\prime}$ of $\operatorname{pr}_{L}: \Sigma \rightarrow L,\left.\mathcal{N}(-\sigma)\right|_{L^{\prime}} \cong N_{L^{\prime} / X}(-1)$. Since $\left([X],[\Sigma],\left[L^{\prime}\right]\right)$ is also in $W^{\mathrm{o}}$, by Lemma 6.3, part iv, $h^{1}\left(L^{\prime}, N_{L^{\prime} / X}(-1)\right)=0$. Therefore $R^{1}\left(\operatorname{pr}_{L}\right)_{*} \mathcal{N}(-\sigma)=\{0\}$, i.e. axiom iii holds.

By Remark 4.4, part ii, $\zeta^{*} T_{\mathrm{ev}} \cong\left(\operatorname{pr}_{L}\right)_{*} \mathcal{N}(-\sigma)$. Part of the long exact sequence of higher direct images associated to the twist by $\mathcal{O}_{\Sigma}(-L)$ of the short exact sequence above is,

$$
\left(\operatorname{pr}_{L}\right)_{*} \operatorname{pr}_{L}^{*} T_{L}(-\sigma(L)) \rightarrow\left(\operatorname{pr}_{L}\right)_{*} \mathcal{N}(-\sigma(L)) \rightarrow\left(\operatorname{pr}_{L}\right)_{*} N_{\Sigma / X}(-\sigma(L)) \rightarrow R^{1}\left(\operatorname{pr}_{L}\right)_{*} \operatorname{pr}_{L}^{*} T_{L}(-\sigma(L)) \rightarrow 0 .
$$

For each fiber $L^{\prime}$ of $\operatorname{pr}_{L},\left.T_{L}(-\sigma(L))\right|_{L^{\prime}} \cong \mathcal{O}_{L^{\prime}}(-1)$. Therefore $\left(\operatorname{pr}_{L}\right)_{*} \operatorname{pr}_{L}^{*} T_{L}(-\sigma(L))=\{0\}$ and $R^{1}\left(\operatorname{pr}_{L}\right)_{*} \operatorname{pr}_{L}^{*} T_{L}(-\sigma(L))=\{0\}$, i.e. $\left(\operatorname{pr}_{L}\right)_{*} \mathcal{N}(-\sigma(L)) \cong\left(\operatorname{pr}_{L}\right)_{*} N_{\Sigma / X}(-\sigma(L))$.

Let $p^{\prime} \in L$ be a closed point and denote $L^{\prime}=\operatorname{pr}_{L}^{-1}\left\{p^{\prime}\right\}$. Since $R^{1}\left(\operatorname{pr}_{L}\right)_{*} \operatorname{pr}_{L}^{*} T_{L}\left(-L-L^{\prime}\right)=$ $\{0\}$ and $R^{1}\left(\operatorname{pr}_{L}\right)_{*} \mathcal{N}\left(-L-L^{\prime}\right)=\{0\}$, by the long exact sequence of higher direct images, also $R^{1}\left(\operatorname{pr}_{L}\right)_{*} N_{\Sigma / X}\left(-L-L^{\prime}\right)=\{0\}$. By the Leray spectral sequence, $h^{1}\left(\Sigma, N_{\Sigma / X}\left(-L-L^{\prime}\right)\right)=h^{1}\left(L,\left(\operatorname{pr}_{L}\right)_{*}\right.$ $\left.\left(N_{\Sigma / X}(-\sigma(L))\right)\left(-p^{\prime}\right)\right)$. By Lemma 6.3, part iii, $h^{1}\left(\Sigma, N_{\Sigma / X}\left(-L-L^{\prime}\right)\right)=0$, so also $h^{1}\left(L,\left(\operatorname{pr}_{L}\right)_{*}\right.$ $\left.\left(N_{\Sigma / X}(-\sigma(L))\right)\left(-p^{\prime}\right)\right)=0$. By Grothendieck's lemma, $\left(\operatorname{pr}_{L}\right)_{*} N_{\Sigma / X}(-\sigma(L))$ is a direct sum of line bundles of degree $\geqslant 0$, i.e. it is generated by global sections. Therefore axiom iv is satisfied. Finally, $\sigma^{*} \mathcal{O}_{\Sigma}(\sigma(L)) \cong \mathcal{O}_{L}$, so axiom $\mathrm{v}$ is satisfied.

Proposition 6.5. If either

i) $d=1$ and $n \geqslant 4$, or

ii) $d \geqslant 2$ and $n+1 \geqslant d^{2}$,

then $\operatorname{pr}_{F(\mathcal{X})}: W^{\mathrm{o}} \rightarrow F(\mathcal{X})$ is dominant. Therefore, for a general pair $([X],[L]) \in F(\mathcal{X}), L$ is a twistable line on $X$.

Proof. By Lemma 6.3, part v, it suffices to prove that $W^{\mathrm{o}}$ is nonempty. 


\section{J. HARRIS AND J. STARR}

i) If $d=1$ and $n \geqslant 4$, then for any quadric surface $\Sigma \subset \mathbb{P}^{n}$ and any hyperplane $X \subset \mathbb{P}^{n}$ containing $\operatorname{span}(\Sigma), \partial$ is an isomorphism so $([X],[\Sigma],[L])$ is in $W^{\circ}$ for any line $L \subset \Sigma$.

ii) Next suppose that $d \geqslant 2$. Denote by $I_{d}$ the set

$$
I_{d}=\{(i, j) \in \mathbb{Z} \times \mathbb{Z}: 0 \leqslant i, j \leqslant d-1, i+j \geqslant 3\},
$$

which has $d^{2}-4$ elements. Denote by

$$
\left(Y_{0}, Y_{1}, Y_{2}, Y_{3}\right) \cup\left(X_{i, j}\right)_{(i, j) \in I_{d}} \cup\left(Z_{m}: m=1, \ldots, n+1-d^{2}\right)
$$

a basis of $H^{0}\left(\mathbb{P}^{n}, \mathcal{O}_{\mathbb{P}^{n}}(1)\right)$, i.e. a basis of homogeneous coordinates on $\mathbb{P}^{n}$. Denote by $\Sigma \subset \mathbb{P}^{n}$ the smooth quadric surface with ideal

$$
I_{\Sigma}=\left\langle Y_{0} Y_{3}-Y_{1} Y_{2}\right\rangle+\left\langle X_{i, j} \mid(i, j) \in I_{d}\right\rangle+\left\langle Z_{m} \mid m=1, \ldots, n+1-d^{2}\right\rangle .
$$

This is the image of the closed immersion $f: \mathbb{P}^{1} \times \mathbb{P}^{1} \rightarrow \mathbb{P}^{n}$,

$$
\left(\left[U_{0}: U_{1}\right],\left[V_{0}: V_{1}\right]\right) \mapsto\left[U_{0} V_{0}: U_{0} V_{1}: U_{1} V_{0}: U_{1} V_{1}: 0: \cdots: 0\right] .
$$

For each $(i, j) \in I_{d}$, denote $k=\min (i, j)$, denote $i^{\prime}=i-k$, and denote $j^{\prime}=j-k$. Denote by $X \subset \mathbb{P}^{n}$ the hypersurface with defining equation

$$
F=\left(Y_{0} Y_{3}-Y_{1} Y_{2}\right) Y_{3}^{d-2}+\sum_{(i, j) \in I_{d}} Y_{0}^{k} Y_{1}^{i^{\prime}} Y_{2}^{j^{\prime}} Y_{3}^{d+k-i-j-1} X_{i, j}
$$

Clearly $\Sigma \subset X$. The claim is that $\partial F: \mathbb{C}^{n+1} \rightarrow H^{0}\left(\Sigma, \mathcal{O}_{\Sigma}(d-1)\right)$ is surjective. By construction,

$$
\frac{\partial F}{\partial Y_{0}} \mapsto U_{1}^{d-1} V_{1}^{d-1}, \frac{\partial F}{\partial Y_{1}} \mapsto U_{1}^{d-1} V_{0} V_{1}^{d-2}, \frac{\partial F}{\partial Y_{2}} \mapsto U_{0} U_{1}^{d-2} V_{1}^{d-1}, \frac{\partial F}{\partial Y_{3}} \mapsto U_{0} U_{1}^{d-2} V_{0} V_{1}^{d-2} .
$$

For each $(i, j) \in I_{d}$,

$$
\frac{\partial F}{\partial X_{i, j}} \mapsto U_{0}^{i} U_{1}^{d-1-i} V_{0}^{j} V_{1}^{d-1-j}
$$

Since the partial derivatives of the form $\partial F / \partial Y_{i}$ give the terms $U_{0}^{i} U_{1}^{d-1-i} V_{0}^{j} V_{1}^{d-1-j}$ with $(i, j)=$ $(0,0),(0,1),(1,0)$, and $(1,1)$, and since these are precisely the pairs $(i, j)$ not contained in $I_{d}, \partial F$ is surjective. Therefore, for every line $L \subset \Sigma,([X],[\Sigma],[L])$ is in $W^{\mathrm{o}}$.

Together with Remark 6.1, Lemma 6.4 and Proposition 6.5 imply the following corollary.

Corollary 6.6. If $X \subset \mathbb{P}^{n}$ is a general hypersurface of degree $d$ and either

i) $d=1$ and $n \geqslant 3$, or

ii) $d \geqslant 2$ and $n+1 \geqslant d^{2}$,

then Hypothesis 4.10 holds.

\section{Base case of the induction for hypersurfaces}

In this section it is proved that if $n \geqslant d^{2}+d+1$ and if $X \subset \mathbb{P}^{n}$ is a general hypersurface of degree $d$, then there exists a morphism $\bar{\zeta}_{1}: \mathbb{P}^{1} \rightarrow \overline{\mathcal{M}}_{0,1}(X, 1)$ that is both very twisting and very positive. This provides the base case for the induction argument of $\S 5$.

Remark 7.1. There is one exceptional case, $d=1$. It will be proved that for $n \geqslant 7$, there is a morphism $\bar{\zeta}_{1}: \mathbb{P}^{1} \rightarrow \overline{\mathcal{M}}_{0,1}\left(\mathbb{P}^{n-1}, 1\right)$ that is both very twisting and very positive. As in Remark 6.1, repeatedly projecting from a point produces a morphism $\bar{\zeta}_{1}: \mathbb{P}^{1} \rightarrow \overline{\mathcal{M}}_{0,1}\left(\mathbb{P}^{n-1}, 1\right)$ that is very twisting and very positive for all $n \geqslant 3$. 


\section{RATIONAL CURVES ON HYPERSURFACES, II}

The techniques in this section are the same as those of $\S 6$. Proposition 6.5 is proved by finding a single degree- $d$ polynomial $F$ on $\mathbb{P}^{n}$, vanishing on some quadric surface $\Sigma$, and such that

$$
\partial_{F, \Sigma}: \mathbb{C}^{n+1} \longrightarrow H^{0}\left(\Sigma, \mathcal{O}_{\Sigma}(d-1)\right)
$$

is surjective. In this section, the role of $L \subset X$ is replaced by a rational normal curve $C_{0} \subset X$ of degree $k \leqslant n$ (in the end, only the case $k=2 d$ will be needed). The role of the quadric surface is replaced by a rational normal scroll $\Sigma$ of degree $2 k-1$ such that $C_{0} \subset \Sigma \subset X$. The cohomology vanishing results of the last section are replaced by the vanishing of $h^{i}\left(\Sigma, N_{\Sigma / X}\left(-C_{0}-2 L\right)\right)$ for $i>0$, where $L$ is a line of the ruling of $\Sigma$. The computation in this section will be to find a single degree- $d$ polynomial $F$ on $\mathbb{P}^{n}$, vanishing on $\Sigma$, and such that the image, $W$, of the map

$$
\partial_{F, \Sigma}: \mathbb{C}^{n+1} \longrightarrow H^{0}\left(\Sigma, \mathcal{O}_{\Sigma}(d-1)\right)
$$

has the property that the induced map

$$
W \otimes H^{0}\left(\Sigma, \mathcal{O}_{\Sigma}((k-3) L)\right) \longrightarrow H^{0}\left(\Sigma, \mathcal{O}_{\Sigma}(d-1) \otimes \mathcal{O}_{\Sigma}((k-3) L)\right)
$$

is surjective. A similar polynomial $F$ to that of the last section satisfies this condition.

\subsection{Generating linear systems on $\mathbb{F}_{1}$}

In the last section, the relevant surface was the Hirzebruch surface $\mathbb{F}_{0}=\mathbb{P}^{1} \times \mathbb{P}^{1}$ embedded as a quadric surface. In this section, the relevant surface is the Hirzebruch surface $\mathbb{F}_{1}$ embedded as a rational normal scroll of degree $2 k-1$. The projective model of $\mathbb{F}_{1}$ used here is,

$$
\mathbb{F}_{1}=\left\{\left(\left[T_{0}: T_{1}\right],\left[T_{0} U: T_{1} U: V\right]\right) \in \mathbb{P}^{1} \times \mathbb{P}^{2} \mid T_{0}\left(T_{1} U\right)=T_{1}\left(T_{0} U\right)\right\} .
$$

In the equation above, ' $T_{0} U$ ' and ' $T_{1} U$ ' are just names of homogeneous coordinates on $\mathbb{P}^{2}$ (although the term $U$ does have a meaning described below). Denote by $\operatorname{pr}_{\mathbb{P}^{1}}: \mathbb{F}_{1} \rightarrow \mathbb{P}^{1}$ and $\operatorname{pr}_{\mathbb{P}^{2}}: \mathbb{F}_{1} \rightarrow \mathbb{P}^{2}$ the projection morphisms. Denote by $\mathcal{O}_{\mathbb{F}_{1}}(F)$ the invertible sheaf $\mathrm{pr}_{\mathbb{P}^{1}}^{*} \mathcal{O}_{\mathbb{P}^{1}}$ and by $\mathcal{O}_{\mathbb{F}_{1}}(E+F)$ the invertible sheaf $\operatorname{pr}_{\mathbb{P}^{2}}^{*} \mathcal{O}_{\mathbb{P}^{2}}$. The invertible sheaf $\mathcal{O}_{\mathbb{F}_{1}}(E)$ is associated to the directrix $E \subset \mathbb{F}_{1}$. (This explains the terminology $T_{0} U$ and $T_{1} U ; U$ is a nonzero element of $H^{0}\left(\mathbb{F}_{1}, \mathcal{O}_{\mathbb{F}_{1}}(E)\right.$ ), and $T_{0} U$ and $T_{1} U$ are the products of $U$ with the two global sections $T_{0}$ and $T_{1}$ of $H^{0}\left(\mathbb{F}_{1}, \mathcal{O}_{\mathbb{F}_{1}}(F)\right)$.)

The invertible sheaves $\mathcal{O}_{\mathbb{F}_{1}}(E+F)$ and $\mathcal{O}_{\mathbb{F}_{1}}(F)$ generate the Picard group of $\mathbb{F}_{1}$, thus motivating the notation

$$
\mathcal{O}(a, b):=\mathcal{O}_{\mathbb{F}_{1}}(a(E+F)+b F) .
$$

The divisors $E+F$ and $F$ are each nef, but not ample. Therefore they generate the nef cone; an invertible sheaf $\mathcal{O}(a, b)$ is nef if and only if $0 \leqslant a, b$, and it is ample if and only if $0<a, b$.

Let $\mathcal{O}(a, b)$ be a nef invertible sheaf, and let $W \subset H^{0}\left(\mathbb{F}_{1}, \mathcal{O}(a, b)\right)$ be a linear system. Let $c \geqslant 0$ be an integer.

Definition 7.2. The linear system $W$ is a $c$-generating linear system if the associated map

$$
\mu_{W, c}: W \otimes H^{0}\left(\mathbb{F}_{1}, \mathcal{O}(0, c)\right) \rightarrow H^{0}\left(\mathbb{F}_{1}, \mathcal{O}(a, b+c)\right)
$$

is surjective.

When is $W$ a $c$-generating linear system?

Notation 7.3. For each integer $i$, denote

$$
r(i)=\left\lfloor\frac{b+i-1}{c+1}\right\rfloor .
$$




\section{J. HARRIS AND J. STARR}

Denote by $\beta_{d}, \beta_{r}, \alpha_{d}$ and $\alpha_{r}$ the unique integers such that $0 \leqslant \alpha_{r}, \beta_{r}<c+1$ and $b-1=\beta_{d}(c+1)+\beta_{r}$, $a+b-1=\alpha_{d}(c+1)+\alpha_{r}$. Denote by $N(a, b, c)$ the integer,

$$
\begin{aligned}
N(a, b, c) & =\sum_{i=0}^{a}(r(i)+2) \\
& =2 a+2+ \begin{cases}\beta_{d}\left(c+1-\beta_{r}\right)+\alpha_{d}\left(1+\alpha_{r}\right)+\left(\alpha_{d}-\beta_{d}-1\right)(c+1), & \alpha_{d}>\beta_{d}, \\
\beta_{d}\left(\alpha_{r}-\beta_{r}+1\right), & \alpha_{d}=\beta_{d} .\end{cases}
\end{aligned}
$$

Denote by $W_{0}(a, b, c) \subset H^{0}\left(\mathbb{F}_{1}, \mathcal{O}(a, b)\right)$ the linear system,

$$
\begin{aligned}
W_{0}(a, b, c)= & \operatorname{span}\left\{U^{i} V^{a-i} T_{0}^{(b+i)-j(c+1)} T_{1}^{j(c+1)} \mid i=0, \ldots, a, j=0, \ldots, r(i)\right\} \\
& +\operatorname{span}\left\{U^{i} V^{a-i} T_{1}^{b+i} \mid i=0, \ldots, a\right\} .
\end{aligned}
$$

Lemma 7.4. The linear system $W_{0}(a, b, c)$ is a c-generating linear system of dimension $N(a, b, c)$.

Proof. For each pair of nonnegative integers $a^{\prime}, b^{\prime}$ there is a decreasing filtration on $H^{0}\left(\mathbb{F}_{1}, \mathcal{O}\left(a^{\prime}, b^{\prime}\right)\right)$,

$$
F^{i} H^{0}\left(\mathbb{F}_{1}, \mathcal{O}\left(a^{\prime}, b^{\prime}\right)\right)=H^{0}\left(\mathbb{F}_{1}, \mathcal{O}\left(a^{\prime}, b^{\prime}\right)(-i E)\right) \cong H^{0}\left(\mathbb{F}_{1}, \mathcal{O}\left(a^{\prime}-i, b^{\prime}\right)\right) .
$$

For any linear system $W \subset H^{0}\left(\mathbb{F}_{1}, \mathcal{O}(a, b)\right)$, there is an induced filtration $F^{i} W=F^{i} \cap W$. The multiplication map $\mu_{W, c}$ respects the filtrations on $W$ and on $H^{0}\left(\mathbb{F}_{1}, \mathcal{O}(a, b+c)\right)$. Hence $\mu_{W, c}$ is surjective if every associated graded map

$$
\operatorname{gr}^{i} \mu_{W, c}: \operatorname{gr}^{i} W \otimes H^{0}\left(\mathbb{F}_{1}, \mathcal{O}(0, c)\right) \rightarrow \operatorname{gr}^{i} H^{0}\left(\mathbb{F}_{1}, \mathcal{O}(a, b+c)\right)
$$

is surjective. Now $\operatorname{dim}(W)$ is the sum of all terms $\operatorname{dim}\left(\operatorname{gr}^{i} W\right)$. For each $i$, what is the minimum possible dimension of a vector subspace $W^{i} \subset \operatorname{gr}^{i} H^{0}\left(\mathbb{F}_{1}, \mathcal{O}(a, b)\right)$ such that the associated map

$$
\operatorname{gr}^{i} \mu_{W^{i}, c}: W^{i} \otimes H^{0}\left(\mathbb{F}_{1}, \mathcal{O}(0, c)\right) \longrightarrow \operatorname{gr}^{i} H^{0}\left(\mathbb{F}_{1}, \mathcal{O}(a, b+c)\right)
$$

is surjective?

The associated graded pieces of $\mathcal{O}\left(a^{\prime}, b^{\prime}\right)$ are,

$$
\operatorname{gr}^{i} H^{0}\left(\mathbb{F}_{1}, \mathcal{O}\left(a^{\prime}, b^{\prime}\right)\right) \cong \begin{cases}H^{0}\left(E, \mathcal{O}_{E}\left(b^{\prime}+i\right)\right), & 0 \leqslant i \leqslant a^{\prime} \\ \{0\}, & i>a^{\prime} .\end{cases}
$$

Let $W^{i} \subset H^{0}\left(E, \mathcal{O}_{E}(b+i)\right)$ be a linear system such that the multiplication map

$$
\operatorname{gr}^{i} \mu_{W^{i}, c}: W^{i} \otimes H^{0}\left(E, \mathcal{O}_{E}(c)\right) \longrightarrow H^{0}\left(E, \mathcal{O}_{E}(b+c+i)\right)
$$

is surjective. Counting dimensions on the left and right sides of the equation, $\operatorname{dim}\left(W^{i}\right) \cdot(c+1) \geqslant$ $(b+c+i+1)$, i.e.

$$
\operatorname{dim}\left(W^{i}\right) \geqslant\left\lfloor\frac{b+i-1}{c+1}\right\rfloor+2=r(i)+2 .
$$

The linear system $W^{i}=\operatorname{gr}^{i} W_{0}(a, b, c)$, which is generated by the set of monomials

$$
\left\{U^{i} V^{a-i} T_{0}^{(b+i)-j(c+1)} T_{1}^{j(c+1)} \mid j=0, \ldots, r(i)\right\} \cup\left\{U^{i} V^{a-i} T_{1}^{b+i}\right\},
$$

has the property that $W^{i} \otimes H^{0}\left(E, \mathcal{O}_{E}(c)\right) \rightarrow H^{0}\left(E, \mathcal{O}_{E}(b+c+i)\right)$ is surjective, and $\operatorname{dim}\left(W^{i}\right)=$ $r(i)+2$. So $W_{0}(a, b, c)$ is a $c$-generating linear system of dimension,

$$
2 a+2+\sum_{i=0}^{a}\left\lfloor\frac{b+i-1}{c+1}\right\rfloor=N(a, b, c) .
$$

Moreover, this is the minimum dimension among $c$-generating linear systems for which each map $\operatorname{gr}^{i} \mu_{W^{i}, c}$ is surjective. 


\section{RATIONAL CURVES ON HYPERSURFACES, II}

Notation 7.5. Denote by $S$ the Cox homogeneous coordinate ring of $\mathbb{F}_{1}$, i.e.

$$
S:=\bigoplus_{(a, b) \in \mathbb{Z}^{2}} H^{0}\left(\mathbb{F}_{1}, \mathcal{O}(a, b)\right)=\mathbb{C}\left[T_{0}, T_{1}, U, V\right] .
$$

This is a $\mathbb{Z}^{2}$-graded ring, where $\operatorname{deg}\left(T_{0}\right)=\operatorname{deg}\left(T_{1}\right)=(0,1), \operatorname{deg}(V)=(1,0)$ and $\operatorname{deg}(U)=(1,-1)$. For every $(a, b) \in \mathbb{Z}^{2}, S_{(a, b)}=H^{0}\left(\mathbb{F}_{1}, \mathcal{O}(a, b)\right)$. Denote by deg $: S \rightarrow \mathbb{Z} \cup\{-\infty\}$ the total degree defined by $\operatorname{deg}(M)=a+b$ for all elements $M \in S_{(a, b)}$. Denote by $\prec$ the graded lexicographical monomial order on $S$ that refines the grading by total degree by $U>V>T_{0}>T_{1}$. For every linear system $W \subset H^{0}\left(\mathbb{F}_{1}, \mathcal{O}(a, b)\right)$ denote by $\operatorname{IN}(W)$ the linear system generated by the initial terms of $W$.

Lemma 7.6. If the linear system $\operatorname{IN}(W)$ contains $W_{0}(a, b, c)$, then $W$ is a c-generating linear system.

Proof. The linear system of initial terms of Image $\left(\mu_{W, c}\right)$ satisfies

$$
\operatorname{IN}(W) \cdot S_{(0, c)} \subset \operatorname{IN}\left(\operatorname{Image}\left(\mu_{W, c}\right)\right) .
$$

Since $\operatorname{IN}(W)$ contains $W_{0}(a, b, c)$,

$$
W_{0}(a, b, c) \cdot S_{(0, c)} \subset \mathrm{IN}\left(\operatorname{Image}\left(\mu_{W, c}\right)\right) .
$$

By Lemma 7.4, $W_{0}(a, b, c) \cdot S_{(0, c)}=S_{(a, b+c)}$. Hence $\operatorname{IN}\left(\operatorname{Image}\left(\mu_{W, c}\right)\right)=S_{(a, b+c)}$, and therefore $\operatorname{Image}\left(\mu_{W, c}\right)=S_{(a, b+c)}$.

Remark 7.7. The most important case is $a=d-1, b=(d-1)(k-1)$ and $c=k-3$ for positive integers $d \geqslant 1$ and $k \geqslant 3$ ( $d$ will be the degree of the hypersurface $X \subset \mathbb{P}^{n}$, and $k$ will be the degree of the curve $\left.C_{0} \subset X\right)$. In particular, if $d \geqslant 2$ and $k \geqslant 2 d$, then $b-1=(d-1)(k-2)+d-2$, $a+b-1=(d-1)(k-2)+2 d-3$ and $0 \leqslant d-2,2 d-3 \leqslant k-3$. Hence $r(i)=d-1$ for $i=0, \ldots, a$ and $N(a, b, c)=d(d+1)=d^{2}+d$. Moreover, $\min _{k \geqslant 3} N(d-1,(d-1)(k-1),(k-3))=d^{2}+d$. This is the origin of the term ' $d{ }^{2}+d$ ' in Theorem 1.1 .

\subsection{Cohomology results}

Notation 7.8. Let $N_{d}, \mathbb{P}^{N_{d}}$, and $\mathcal{X} \subset \mathbb{P}^{N_{d}} \times \mathbb{P}^{n}$ be as in Notation 6.2. Let $k$ be any integer with $3 \leqslant k \leqslant n / 2$ (only the case $k=2 d$ will be used later). Let $\mathcal{R}^{k}\left(\mathbb{P}^{n}\right) \subset{\text { Hilb } \mathbb{P}^{n}}^{k t+1}$ denote the open subscheme parametrizing curves $C_{0} \subset \mathbb{P}^{n}$ that are projectively equivalent to a degree- $k$ rational normal curve $C_{0} \subset \mathbb{P}^{k} \subset \mathbb{P}^{n}$. Let $\mathcal{R}^{k}(\mathcal{X}) \subset \mathbb{P}^{N_{d}} \times \mathcal{R}^{k}\left(\mathbb{P}^{n}\right)$ denote the parameter space for pairs ([X], $\left.\left[C_{0}\right]\right)$ such that $C_{0} \subset X$. Let $Q(t)=\frac{1}{2}(t+1)((2 k-1) t+2)$ denote the Hilbert polynomial of a rational normal scroll of degree $2 k-1$ in $\mathbb{P}^{2 k}$. Let $\mathcal{U} \subset \operatorname{Hilb}_{\mathbb{P n}}^{Q(t)}$ denote the open subscheme parametrizing closed subschemes $\Sigma \subset \mathbb{P}^{n}$ that are projectively equivalent to a rational normal scroll of degree $2 k-1$ in $\mathbb{P}^{2 k} \subset \mathbb{P}^{n}$ and that are abstractly isomorphic to $\mathbb{F}_{1}$. Let $\mathcal{V} \subset \mathcal{U} \times \mathcal{R}^{k}\left(\mathbb{P}^{n}\right)$ denote the parameter space of pairs $\left([\Sigma],\left[C_{0}\right]\right)$ such that $C_{0} \subset \Sigma$ and such that, via the isomorphism of $\Sigma \cong \mathbb{F}_{1}$, the invertible sheaf of $C_{0}$ is $\mathcal{O}(1,0)$. Let $\mathcal{W} \subset \mathbb{P}^{N_{d}} \times \mathcal{U} \times \mathcal{R}^{k}\left(\mathbb{P}^{n}\right)$ denote the parameter space for triples $\left([X],[\Sigma],\left[C_{0}\right]\right)$ where $\left([\Sigma],\left[C_{0}\right]\right)$ is in $\mathcal{V}$ and where $\Sigma \subset X$.

Observe that $\mathcal{R}^{k}\left(\mathbb{P}^{n}\right)$ is a homogeneous space of $\mathrm{PGL}_{n+1}$, and therefore is smooth and connected. Observe that the projection $\mathcal{R}^{k}(\mathcal{X}) \rightarrow \mathcal{R}^{k}\left(\mathbb{P}^{n}\right)$ is a projective bundle of relative dimension $N_{d}-(k d+1)$. Observe that the projection map $\mathcal{V} \rightarrow \mathcal{U}$ factors as an open subset (with nonempty fibers) of a projective bundle over $\mathcal{U}$ of relative dimension 2 (more precisely, every fiber is isomorphic to the $\mathbb{A}^{2}$ of irreducible curves in the linear system $\left.|\mathcal{O}(1,0)|\right)$. Observe that the projection map $\mathcal{W} \rightarrow \mathcal{V}$ is a projective bundle of relative dimension $N_{d}-Q(d)$.

For each triple $\left([X],[\Sigma],\left[C_{0}\right]\right) \in \mathcal{W}$, define $\partial_{X, \Sigma}: \mathbb{C}^{n+1} \rightarrow H^{0}\left(\Sigma, \mathcal{O}_{\Sigma}(d-1)\right)$ as in $\S 6$. More precisely, denote $\mathcal{E}=\mathcal{O}_{W}^{\oplus(n+1)}$. Denote by $\mathcal{G}$ the unique quotient of $H^{0}\left(\mathbb{P}^{n}, \mathcal{O}_{\mathbb{P}^{n}}(d-1)\right) \otimes_{\mathbb{C}} \mathcal{O}_{\mathcal{U}}$ 


\section{J. HARRIS AND J. STARR}

that is locally free and whose fiber at each point $\Sigma$ is the quotient

$$
H^{0}\left(\mathbb{P}^{n}, \mathcal{O}_{\mathbb{P}^{n}}(d-1)\right) \longrightarrow H^{0}\left(\Sigma, \mathcal{O}_{\Sigma}(d-1)\right) .
$$

Denote by $\mathcal{F}$ the locally free $\mathcal{O}_{\mathcal{W}}$-modules

$$
\operatorname{pr}_{\mathbb{P}^{N}}^{*}\left(\mathcal{O}_{\mathbb{P}^{N_{d}}}(1)\right) \otimes \operatorname{pr}_{\mathcal{U}}^{*} \mathcal{G} .
$$

Then there is a map of $\mathcal{O}_{\mathcal{W}}$-modules $\partial: \mathcal{E} \rightarrow \mathcal{F}$ whose fiber at each point $\left([X],[\Sigma],\left[C_{0}\right]\right)$ is the map $\partial_{X, \Sigma}$. Denote by $\mathcal{W}^{0} \subset \mathcal{W}$ the open subscheme parametrizing points $\left([X],[\Sigma],\left[C_{0}\right]\right)$ such that

$$
\operatorname{Image}\left(\partial_{X, \Sigma}\right) \subset H^{0}\left(\Sigma,\left.\mathcal{O}_{\mathbb{P}^{n}}(d-3)\right|_{\Sigma}\right)
$$

is a $(k-3)$-generating linear system.

Let $\Sigma \subset \mathbb{P}^{2 k} \subset \mathbb{P}^{n}$ be a rational normal surface scroll of degree $2 k-1$, and let $f: \mathbb{F}_{1} \rightarrow \Sigma$ be an isomorphism. For each pair of nonnegative integers $(a, b)$, denote by $N(a, b)$ the locally free $\mathcal{O}_{\mathbb{F}_{1}}$-module

$$
N(a, b)=f^{*}\left(\left.N_{\Sigma / \mathbb{P}^{n}} \otimes \mathcal{O}_{\mathbb{P}^{n}}(-1)\right|_{\Sigma}\right) \otimes \mathcal{O}(a, b),
$$

and denote by $N^{\prime}(a, b) \subset N(a, b)$ the subsheaf

$$
N^{\prime}(a, b)=f^{*}\left(\left.N_{\Sigma / \mathbb{P}^{2 k}} \otimes \mathcal{O}_{\mathbb{P}^{n}}(-1)\right|_{\Sigma}\right) \otimes \mathcal{O}(a, b)
$$

LEMMA 7.9.

i) $N^{\prime}(0,0)$ is generated by global sections and $h^{i}\left(\mathbb{F}_{1}, N^{\prime}(0,0)\right)=0$ for $i>0$

ii) $N(0,0)$ is generated by global sections and $h^{i}\left(\mathbb{F}_{1}, N(0,0)\right)=0$ for $i>0$

iii) For every pair of nonnegative integers $(a, b)$ and for every coherent sheaf $\mathcal{F}$ on $\mathbb{F}_{1}$ that is generated by global sections and such that $h^{i}\left(\mathbb{F}_{1}, \mathcal{F}\right)=0$ for $i>0, \mathcal{F}(a, b):=\mathcal{F} \otimes \mathcal{O}(a, b)$ is generated by global sections and $h^{i}\left(\mathbb{F}_{1}, \mathcal{F}(a, b)\right)=0$ for $i>0$.

In particular, for every pair of nonnegative integers $(a, b), N(a, b)$ (respectively $N^{\prime}(a, b)$ ) is generated by global sections and $h^{i}\left(\mathbb{F}_{1}, N(a, b)\right)=0$ for $i>0$ (respectively $h^{i}\left(\mathbb{F}_{1}, N^{\prime}(a, b)\right)=0$ for $i>0$ ).

Proof. i) The morphism $\operatorname{pr}_{\mathbb{P}^{1}}: \mathbb{F}_{1} \rightarrow \mathbb{P}^{1}$ is isomorphic over $\mathbb{P}^{1}$ to projection from the projective bundle

$$
\mathbb{F}_{1} \cong \mathbb{P}\left(\mathcal{O}_{\mathbb{P}^{1}}(-(k-1)) \oplus \mathcal{O}_{\mathbb{P}^{1}}(-k)\right) .
$$

Under this isomorphism the invertible sheaf $\mathcal{O}(1, k-1)$ on $\Sigma$ corresponds to the invertible sheaf $\mathcal{O}(1)$ on $\mathbb{P}\left(\mathcal{O}_{\mathbb{P}^{1}}(-(k-1)) \oplus \mathcal{O}_{\mathbb{P}^{1}}(-k)\right)$ where $\mathcal{O}(1)$ is the universal invertible quotient of $\operatorname{pr}_{\mathbb{P}^{1}}^{*}\left(\mathcal{O}_{\mathbb{P}^{1}}(k-1)\right.$ $\left.\oplus \mathcal{O}_{\mathbb{P}^{1}}(k)\right)$. Up to projective equivalence, the morphism $f: \mathbb{F}_{1} \rightarrow \mathbb{P}^{2 k}$ is the closed immersion given by the complete linear system of $\mathcal{O}(1)$; in particular, $f^{*} \mathcal{O}_{\mathbb{P}^{2 k}}(1) \cong \mathcal{O}(1)$. Using this isomorphism, there is a short exact sequence of $\mathcal{O}_{\mathbb{F}_{1}}$-modules,

$$
0 \longrightarrow \operatorname{pr}_{\mathbb{P}^{1}}^{*} T_{\mathbb{P}^{1}} \longrightarrow \operatorname{pr}_{\mathbb{P}^{1}}^{*}\left(\mathcal{O}_{\mathbb{P}^{1}}(1)^{\oplus(2 k-1)}\right) \otimes f^{*} \mathcal{O}_{\mathbb{P}^{2 k}}(1) \longrightarrow f^{*} N_{\Sigma / \mathbb{P}^{2 k}} \longrightarrow 0 .
$$

Twisting by $f^{*} \mathcal{O}_{\mathbb{P}^{2 k}}(-1), N^{\prime}(0,0)$ is a quotient of $\operatorname{pr}_{\mathbb{P}^{1}}^{*}\left(\mathcal{O}_{\mathbb{P}^{1}}(1)^{\oplus(2 k-1)}\right)$. Hence $N^{\prime}(0,0)$ is generated by global sections. Also,

$$
\left(\operatorname{pr}_{\mathbb{P}^{1}}\right)_{*}\left(\operatorname{pr}_{\mathbb{P}^{1}}^{*} T_{\mathbb{P}^{1}} \otimes f^{*} \mathcal{O}_{\mathbb{P}^{2 k}}(-1)\right)=\{0\}, \quad R^{1}\left(\operatorname{pr}_{\mathbb{P}^{1}}\right)_{*}\left(\operatorname{pr}_{\mathbb{P}^{1}}^{*} T_{\mathbb{P}^{1}} \otimes f^{*} \mathcal{O}_{\mathbb{P}^{2 k}}(-1)\right)=\{0\} .
$$

Twisting the short exact sequence by $f^{*} \mathcal{O}_{\mathbb{P}^{2 k}}(-1)$ and forming the associated long exact sequence of higher direct images, $R^{1}\left(\operatorname{pr}_{\mathbb{P}^{1}}\right)_{*}\left(f^{*} N_{\Sigma / \mathbb{P}^{2 k}}(-1)\right)=\{0\}$, and $\left(\operatorname{pr}_{\mathbb{P}^{1}}\right)_{*}\left(f^{*} N_{\Sigma / \mathbb{P}^{2 k}}(-1)\right) \cong \mathcal{O}_{\mathbb{P}^{1}}(1)^{\oplus(2 k-1)}$. Computing the cohomology of $N^{\prime}(0,0)$ via the Leray spectral sequence associated to $\mathrm{pr}_{\mathbb{P}^{1}}: \mathbb{F}_{1} \rightarrow \mathbb{P}^{1}$, $h^{i}\left(\mathbb{F}_{1}, N^{\prime}(0,0)\right)=0$ for $i>0$. 


\section{RATIONAL CURVES ON HYPERSURFACES, II}

ii) There is a short exact sequence,

$$
0 \longrightarrow N^{\prime}(0,0) \longrightarrow N(0,0) \longrightarrow \mathcal{O}(0,0)^{\oplus(n-2 k)} \longrightarrow 0 .
$$

By part i, $h^{i}\left(\mathbb{F}_{1}, \mathcal{O}_{\mathbb{F}_{1}}\right)=0$ for $i>0$. Therefore $N(0,0)$ is generated by global sections and $h^{i}\left(\mathbb{F}_{1}, N(0,0)\right)=0$ for $i>0$.

iii) Let $\mathcal{F}$ be a coherent sheaf on $\mathbb{F}_{1}$ such that $\mathcal{F}$ is generated by global sections and such that $h^{i}\left(\mathbb{F}_{1}, \mathcal{F}\right)=0$ for $i>0$. It will be proved by double induction on $(a, b)$ that, for every pair of nonnegative integers $(a, b), \mathcal{F}(a, b)$ is generated by global sections and $h^{i}\left(\mathbb{F}_{1}, \mathcal{F}(a, b)\right)=0$ for $i>0$.

The base case is $b=0$ and is established by induction on $a$. For $a=0$, the result follows by hypothesis. Let $a>0$ and, by way of induction, suppose the result is proved for $a-1$. Let $D \subset \mathbb{F}_{1}$ be a general member of the linear system $|\mathcal{O}(1,0)|$. Then $D$ is a smooth curve isomorphic to $\mathbb{P}^{1}$. Since $D$ is general, there is a short exact sequence,

$$
\left.0 \longrightarrow \mathcal{F}(a-1,0) \longrightarrow \mathcal{F}(a, 0) \longrightarrow \mathcal{F}(a, 0)\right|_{D} \longrightarrow 0 .
$$

The sheaf $\left.\mathcal{F}\right|_{D}$ is generated by global sections, and $\left.\mathcal{O}_{\mathbb{F}_{1}}(a(e+f))\right|_{D} \cong \mathcal{O}_{\mathbb{P}^{1}}(a)$. Hence also $\left.\mathcal{F}(a, 0)\right|_{D}$ is generated by global sections. By the induction assumption, $h^{1}\left(\mathbb{F}_{1}, \mathcal{F}(a-1,0)\right)=0$. By the long exact sequence of cohomology associated to the short exact sequence, every global section of $\left.\mathcal{F}(a, 0)\right|_{D}$ is the image of a global section of $\mathcal{F}(a, 0)$. Hence $\mathcal{F}(a, 0)$ is generated by global sections. A coherent sheaf on $\mathbb{P}^{1}$ that is generated by global sections has no higher cohomology. Combined with the induction assumption and the long exact sequence in cohomology associated to the short exact sequence above, $h^{i}\left(\mathbb{F}_{1}, \mathcal{F}(a, 0)\right)=0$ for $i>0$. Therefore, for every $a>0, \mathcal{F}(a, 0)$ is generated by global sections and $h^{i}\left(\mathbb{F}_{1}, \mathcal{F}(a, 0)\right)=0$ for $i>0$.

Suppose that $b>0$ and suppose the result is proved for $b-1$. Let $L \subset \mathbb{F}_{1}$ be a general fiber of $\operatorname{pr}_{1}$. Then $L$ is smooth and isomorphic to $\mathbb{P}^{1}$. Since $L$ is general, there is a short exact sequence,

$$
\left.0 \longrightarrow \mathcal{F}(a, b-1) \longrightarrow \mathcal{F}(a, b) \longrightarrow \mathcal{F}(a, b)\right|_{L} \longrightarrow 0 .
$$

Via the isomorphism $L \cong \mathbb{P}^{1},\left.\mathcal{O}_{\mathbb{F}_{1}}(a(e+f)+b f)\right|_{L} \cong \mathcal{O}_{\mathbb{P}^{1}}(a)$. By almost identical arguments to those above, $\mathcal{F}(a, b)$ is generated by global sections and $h^{i}\left(\mathbb{F}_{1}, \mathcal{F}(a, b)\right)=0$ for $i>0$.

Let $\left([X],[\Sigma],\left[C_{0}\right]\right)$ be a point in $\mathcal{W}^{\circ}$ and let $f: \mathbb{F}_{1} \rightarrow \Sigma$ be an isomorphism. For each pair of nonnegative integers $(a, b)$, denote $N_{X}(a, b)=f^{*}\left(\left.N_{\Sigma / X} \otimes \mathcal{O}_{\mathbb{P}^{n}}(-1)\right|_{\Sigma}\right) \otimes \mathcal{O}(a, b)$.

\section{LEMMA 7.10.}

i) The hypersurface $X$ is smooth along $\Sigma$.

ii) For each pair of nonnegative integers $(a, b), h^{i}\left(\mathbb{F}_{1}, N_{X}(a, b+k-3)\right)=0$ for $i>0$.

iii) For every line of ruling $L \subset \Sigma$ and every nonnegative integer $a, h^{1}\left(L, N_{L / X}(a-1)\right)=0$.

iv) For every nonnegative integer $a, h^{1}\left(C_{0}, N_{C_{0} / X}(a-2)\right)=0$.

v) The projection morphism $\operatorname{pr}_{\mathbb{P}^{N_{d}}}: \mathcal{W} \rightarrow \mathbb{P}^{N_{d}}$ is smooth at $\left([X],[\Sigma],\left[C_{0}\right]\right)$.

vi) For every line of ruling $L \subset \Sigma$, the projection morphism $\operatorname{pr}_{\mathbb{P}^{N_{d}}}: F(\mathcal{X}) \rightarrow \mathbb{P}^{N_{d}}$ is smooth at $([X],[L])$.

vii) The projection morphism $\operatorname{pr}_{\mathbb{P}^{N_{d}}}: \mathcal{R}^{k}(\mathcal{X}) \rightarrow \mathbb{P}^{N_{d}}$ is smooth at $\left([X],\left[C_{0}\right]\right)$.

viii) The projection morphism $\pi: \mathcal{W} \rightarrow \mathcal{R}^{k}(\mathcal{X})$ is smooth at $\left([X],[\Sigma],\left[C_{0}\right]\right)$.

Proof. i) Since the partial derivatives of a defining equation of $X$ span a $c$-generating linear series, in particular they generate the sheaf $\mathcal{O}_{\Sigma}(d-1)$. Hence, there is no point of $\Sigma$ at which all the partial derivatives vanish. By the Jacobian criterion, $X$ is smooth along $\Sigma$. 


\section{J. HARRIS AND J. STARR}

ii) There is a short exact sequence,

$$
\left.0 \longrightarrow N_{\Sigma / X} \longrightarrow N_{\Sigma / \mathbb{P}^{n}} \longrightarrow N_{X / \mathbb{P}^{n}}\right|_{\Sigma} \longrightarrow 0
$$

Denote $\alpha=a+(d-1)$ and $\beta=b+(d-1)(k-1)$ (these are different from $\alpha_{d}, \alpha_{r}, \beta_{d}$ and $\beta_{r}$ ). There is a short exact sequence,

$$
0 \longrightarrow N_{X}(a, b) \longrightarrow N(a, b) \longrightarrow \mathcal{O}(\alpha, \beta) \longrightarrow 0 .
$$

Since $a, b \geqslant 0$, by Lemma $7.9, h^{i}\left(\mathbb{F}_{1}, N(a, b)\right)=0$ for $i \geqslant 0$. By direct computation, $h^{i}\left(\mathbb{F}_{1}, \mathcal{O}(\alpha, \beta)\right)=$ 0 for $i \geqslant 0$. Hence $h^{2}\left(\mathbb{F}_{1}, N_{X}(a, b)\right)=0$, and $h^{1}\left(\mathbb{F}_{1}, N_{X}(a, b)\right)=0$ if and only if the following map is surjective:

$$
H^{0}\left(\mathbb{F}_{1}, N(a, b)\right) \longrightarrow H^{0}\left(\mathbb{F}_{1}, \mathcal{O}(\alpha, \beta)\right) .
$$

There is the following commutative diagram:

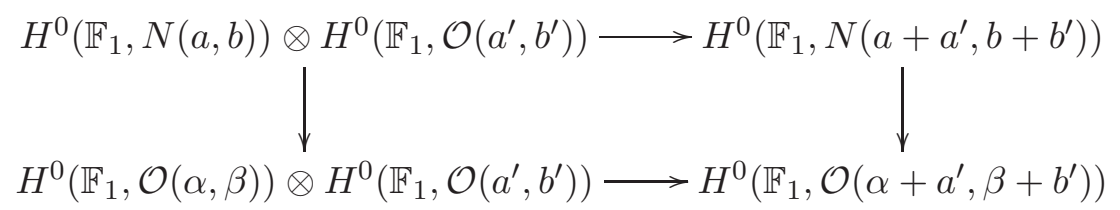

By direct computation the bottom horizontal arrow is surjective if $a^{\prime}, b^{\prime} \geqslant 0$. Hence, if the left vertical arrow is surjective, then also the right vertical arrow is surjective; i.e. if $h^{1}\left(\mathbb{F}_{1}, N_{X}(a, b)\right)=0$ then also $h^{1}\left(\mathbb{F}_{1}, N_{X}\left(a+a^{\prime}, b+b^{\prime}\right)\right)=0$. Thus part ii is reduced to the case $a=0, b=k-3$. In this case the commutative diagram above factors the following commutative diagram

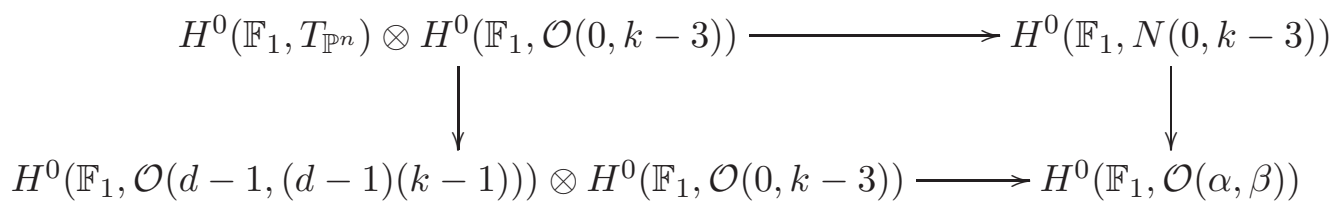

By definition, the composition

$$
H^{0}\left(\mathbb{F}_{1} T_{\mathbb{P} n}\right) \otimes H^{0}\left(\mathbb{F}_{1}, \mathcal{O}(0, k-3)\right) \longrightarrow H^{0}\left(\mathbb{F}_{1}, \mathcal{O}(\alpha, \beta)\right)
$$

is surjective if and only if the triple $\left([X],[\Sigma],\left[C_{0}\right]\right)$ is in $\mathcal{W}^{\circ}$. Since $\left([X],[\Sigma],\left[C_{0}\right]\right)$ is in $\mathcal{W}^{\circ}$, the right vertical arrow is surjective, i.e. $h^{1}\left(\mathbb{F}_{1}, N_{X}(0, k-3)\right)=0$.

iii) There is a short exact sequence,

$$
\left.0 \longrightarrow N_{L / \Sigma}(a-1) \longrightarrow N_{L / X}(a-1) \longrightarrow N_{\Sigma / X}\right|_{L}(a-1) \longrightarrow 0 .
$$

Since $N_{L / \Sigma} \cong \mathcal{O}_{L}$, for all $a \geqslant 0, h^{1}\left(L, N_{L / \Sigma}(a-1)\right)=0$. Therefore it suffices to prove $h^{1}\left(L,\left.N_{\Sigma / X}\right|_{L}(a-1)\right)=0$. Since $\left.\mathcal{O}(a-1, b)\right|_{L} \cong \mathcal{O}_{L}(a-1)$, there is a short exact sequence,

$$
\left.0 \longrightarrow N_{X}(a, k-3) \longrightarrow N_{X}(a, k-2) \longrightarrow N_{\Sigma / X}\right|_{L}(a-1) \longrightarrow 0 .
$$

By part ii, for $a \geqslant 0$ the higher cohomology of the first two terms vanishes. By the long exact sequence in cohomology associated to this short exact sequence, $h^{1}\left(L,\left.N_{\Sigma / X}\right|_{L}(a-1)\right)=0$ for $a \geqslant 0$.

iv) The proof is almost identical to the proof of part iii.

v) By [Kol96, Proposition 2.14.2], the obstruction space for the relative Hilbert scheme $\operatorname{Hilb}_{\mathcal{X} / \mathbb{P}^{N_{d}}}^{Q(t)}$ at the point $([X],[\Sigma])$ is contained in $H^{1}\left(\Sigma, N_{\Sigma / X}\right)$. If $\left([X],[\Sigma],\left[C_{0}\right]\right)$ is in $\mathcal{W}^{\circ}$, then by part ii, $h^{1}\left(\Sigma, N_{\Sigma / X}\right)=h^{1}\left(\mathbb{F}_{1}, N_{X}(1, k-1)\right)=0$. By [Kol96, Theorem 2.10], Hilb $\mathcal{X}_{\mathcal{X} \mathbb{P}^{N_{d}}}^{Q(t)} \rightarrow \mathbb{P}^{N_{d}}$ is smooth 


\section{RATIONAL CURVES ON HYPERSURFACES, II}

at $([X],[\Sigma])$. The projection $\mathcal{W}^{\circ} \rightarrow \operatorname{Hilb}^{Q(t)}\left(\mathcal{X} / \mathbb{P}^{N_{d}}\right)$ is an open subset of a projective bundle, and so is smooth. Therefore the composite morphism $\mathcal{W}^{\circ} \rightarrow \mathbb{P}^{N_{d}}$ is smooth at $\left([X],[\Sigma],\left[C_{0}\right]\right)$.

vi) The proof is very similar to the proof of part $\mathrm{v}$ and uses the vanishing, $h^{1}\left(L, N_{L / X}\right)=0$, which was proved in part iii.

vii) The proof is very similar to the proof of part $\mathrm{v}$ and uses the vanishing, $h^{1}\left(C_{0}, N_{C_{0} / X}\right)=0$, which was proved in part iv.

viii) Since $\mathcal{W}^{\circ} \rightarrow \mathbb{P}^{N_{d}}$ is smooth at $\left([X],[\Sigma],\left[C_{0}\right]\right)$ and since $\mathcal{R}^{k} \rightarrow \mathbb{P}^{N_{d}}$ is smooth at $\left([X],\left[C_{0}\right]\right)$, to prove that $\pi: \mathcal{W}^{\circ} \rightarrow \mathcal{R}^{k}(\mathcal{X})$ is smooth at $\left([X],[\Sigma],\left[C_{0}\right]\right)$, it suffices to check that the derivative map $d \pi: T_{\mathcal{W}^{\circ} / \mathbb{P}^{N_{d}}} \rightarrow \pi^{*} T_{\mathcal{R}^{k}(\mathcal{X}) / \mathbb{P}^{N_{d}}}$ is surjective at $\left([X],[\Sigma],\left[C_{0}\right]\right)$. This reduces to the statement that $H^{0}\left(\Sigma, N_{\Sigma / X}\right) \rightarrow H^{0}\left(C_{0},\left.N_{\Sigma / X}\right|_{C_{0}}\right)$ is surjective. The cokernel is contained in $H^{1}\left(\mathbb{F}_{1}, N_{X}(0, k-1)\right)$. By part iii, $h^{1}\left(C_{0},\left.N_{\Sigma / X}\right|_{C_{0}}\right)=0$, and therefore the derivative $d \pi$ is surjective at $\left([X],[\Sigma],\left[C_{0}\right]\right)$.

Let $\left([X],[\Sigma],\left[C_{0}\right]\right)$ be a point of $\mathcal{W}$. Denote by $\sigma: C_{0} \rightarrow \Sigma$ the inclusion and denote by $\operatorname{pr}_{C_{0}}$ : $\Sigma \rightarrow C_{0}$ the unique projection morphism such that $\sigma$ is a section of $\operatorname{pr}_{C_{0}}$ (via the isomorphism $\Sigma \cong \mathbb{F}_{1}, \operatorname{pr}_{C_{0}}$ corresponds to $\left.\operatorname{pr}_{\mathbb{P}^{1}}\right)$. Denote by $g: \Sigma \rightarrow X$ the inclusion. There is a family of stable maps $\zeta: C_{0} \rightarrow \overline{\mathcal{M}}_{0,1}(X, 1)$,

$$
\zeta=\left(\left(\operatorname{pr}_{C_{0}}: \Sigma \rightarrow C_{0}, \sigma\right), g: \Sigma \rightarrow X\right) .
$$

Lemma 7.11. If $\left([X],[\Sigma],\left[C_{0}\right]\right)$ is in $\mathcal{W}^{\mathrm{o}}$, then $\zeta: C_{0} \rightarrow \overline{\mathcal{M}}_{0,1}(X, 1)$ is very twisting and very positive.

Proof. Very twisting: First the axioms of Definition 4.3 are verified. Since $g \circ \sigma: C_{0} \rightarrow X$ is an embedding, axiom i of Definition 4.3 is satisfied. By Lemma 7.10, part vii, the dimension of the obstruction group of $\overline{\mathcal{M}}_{0,0}(X, k)$ at $\left[g \circ \sigma: C_{0} \rightarrow X\right]$ is 0 , i.e. axiom ii is satisfied.

The proof that axiom iii holds is identical to the argument for axiom iii in the proof of Lemma 6.4, with Lemma 6.3, part iv replaced by Lemma 7.10, part iii.

As in the proof of Lemma $6.4, \zeta^{*} T_{\mathrm{ev}} \cong\left(\operatorname{pr}_{C_{0}}\right)_{*} N_{X}(0, k-1)$. Hence $\zeta^{*} T_{\mathrm{ev}}$ is ample if and only if $h^{1}\left(C_{0}, \zeta^{*} T_{\mathrm{ev}}(-2)\right)=0$. By a Leray spectral sequence argument similar to the one in the proof of Lemma $6.4, h^{1}\left(C_{0}, \zeta^{*} T_{\mathrm{ev}}(-2)\right)=h^{1}\left(\mathbb{F}_{1}, N_{X}(0, k-3)\right)$, which, by Lemma 7.10 , part ii, equals 0 . Therefore $\zeta^{*} T_{\mathrm{ev}}$ is an ample bundle, i.e. axiom iv is satisfied.

Finally, observe that $\sigma^{*} \mathcal{O}_{\Sigma}(\sigma) \cong \mathcal{O}_{C_{0}}(1)$ is ample, i.e. axiom $\mathrm{v}$ is satisfied. Thus $\zeta$ is a very twisting family.

Very positive: Next the axioms of Definition 4.11 are verified. Axioms i, ii and iil follow from axioms i, ii and iii of Definition 4.3, as proved above. There is a short exact sequence,

$$
0 \longrightarrow \zeta^{*} T_{\mathrm{ev}} \longrightarrow \zeta^{*} T_{\overline{\mathcal{M}}_{0,1}(X, 1)} \longrightarrow(g \circ \sigma)^{*} T_{X} \longrightarrow 0 .
$$

It is proved above that $\zeta^{*} T_{\mathrm{ev}}$ is ample. Moreover, by Lemma 7.10, part iv, $N_{C_{0} / X}$ is ample. Of course $T_{C_{0}}$ is ample. Therefore $\left.T_{X}\right|_{C_{0}}$ is ample by Lemma 2.10, part ii. Since the first and last terms in the short exact sequence are ample, by Lemma 2.10, part ii, $\zeta^{*} T_{\overline{\mathcal{M}}_{0,1}(X, 1)}$ is ample. Since $\zeta^{*} \operatorname{pr}^{*} T_{\overline{\mathcal{M}}_{0,0}(X, 1)}$ is a quotient of $\zeta^{*} T_{\overline{\mathcal{M}}_{0,1}(X, 1)}$, by Lemma 2.10, part i, $\zeta^{*} \operatorname{pr}^{*} T_{\overline{\mathcal{M}}_{0,0}(X, 1)}$ is ample, i.e. axiom iv is satisfied.

Finally, $\sigma^{*} \mathcal{O}_{\Sigma}(\sigma) \cong \mathcal{O}_{C_{0}}(1)$, which is ample, i.e. axiom $\mathrm{v}$ is satisfied. Thus $\zeta$ is a very positive family.

\section{Proposition 7.12.}

i) If $d \geqslant 3$ and $n \geqslant d^{2}+d+1$, then for $k=2 d, \mathcal{W}^{\circ} \rightarrow \mathcal{R}^{k}(\mathcal{X})$ is dominant, and $\mathcal{R}^{k}(\mathcal{X}) \rightarrow \mathbb{P}^{N_{d}}$ is dominant.

ii) If $d=1$ or 2 and if $n \geqslant 7$, then for $k=3, \mathcal{W}^{\circ} \rightarrow \mathcal{R}^{3}(\mathcal{X})$ is dominant, and $\mathcal{R}^{3}(\mathcal{X}) \rightarrow \mathbb{P}^{N_{d}}$ is dominant. 


\section{J. HARRIS AND J. STARR}

Proof. By part viii of Lemma 7.10, it suffices to prove that $\mathcal{W}^{\circ}$ is nonempty. We have to find a pair $([X],[\Sigma])$ such that for $a=d-1, b=(d-1)(k-1)$ and for $c=k-3$, we have that the image of the derivative map

$$
d_{X, \Sigma}: H^{0}\left(\mathbb{P}^{n}, T_{\mathbb{P}^{n}}(-1)\right) \rightarrow H^{0}\left(\mathbb{F}_{1}, \mathcal{O}(a, b)\right)
$$

is a $c$-generating linear system.

Recall that $S=\mathbb{C}\left[T_{0}, T_{1}, U, V\right]$ is the $\mathbb{Z}^{2}$-graded Cox homogeneous coordinate ring of $\mathbb{F}_{1}$. Denote by $A_{d}$ the set of $d^{2}+d$ monomials that occur in the linear system $W_{0}(a, b, c)$, i.e.

$$
\begin{aligned}
A_{d}= & \left\{U^{i} V^{d-1-i} T_{0}^{((d-1)(k-1)+i)-j(k-2)} T_{1}^{j(k-2)} \mid i=0, \ldots, d-1, j=1, \ldots, r(i)\right\} \\
& \cup\left\{U^{i} V^{d-1-i} T_{1}^{(d-1)(k-1)+i} \mid i=0, \ldots, d-1\right\}
\end{aligned}
$$

where

$$
r(i)=d-1+\left\lfloor\frac{d-2+i}{k-2}\right\rfloor
$$

i) $d \geqslant 4$ : Suppose that $d \geqslant 4$ and $n \geqslant d^{2}+d+1$. Denote by $B_{d}$ the set of $4 d-1$ monomials,

$$
\begin{aligned}
B_{d}= & \left\{\begin{array}{lllll|lll}
U^{d-1} & & T_{0}^{(d-1) k-(k-2) j} & T_{1}^{(k-2) j} & j=0, \ldots, d-1 &
\end{array}\right\} \\
& \cup\left\{\begin{array}{lllllll}
{ }^{d-2} & V & T_{0}^{(d-1) k-1-(k-2) j} & T_{1}^{(k-2) j} & j=0, \ldots, d-1 &
\end{array}\right\} \\
& \cup\left\{\begin{array}{lllllll}
{ }^{d-3} & V^{2} & T_{0}^{(d-1) k-2-(k-2) j} & T_{1}^{(k-2) j} & j=1, \ldots, d-1 &
\end{array}\right\} \\
& \cup\left\{\begin{array}{llllll}
U^{d-4} & V^{3} & T_{0}^{(d-1) k-3-(k-2) j} & T_{1}^{(k-2) j} & j=1, \ldots, d-1 &
\end{array}\right\}
\end{aligned}
$$

Denote by $C_{d}$ the set of $d^{2}-3 d+1$ monomials $C_{d}=A_{d}-B_{d}$. Denote by

$$
\left\{Y_{0}, \ldots, Y_{k}\right\} \cup\left\{Z_{0}, \ldots, Z_{k-1}\right\} \cup\left\{X_{M} \mid M \in C_{d}\right\} \cup\left\{V_{l} \mid l=1, \ldots, n-\left(d^{2}+d+1\right)\right\}
$$

a basis of $H^{0}\left(\mathbb{P}^{n}, \mathcal{O}_{\mathbb{P}^{n}}(1)\right)$, i.e. a basis of homogeneous coordinates on $\mathbb{P}^{n}$.

Denote by $f: \mathbb{F}_{1} \rightarrow \mathbb{P}^{2 k} \subset \mathbb{P}^{n}$ the morphism mapping $\left(\left[T_{0}: T_{1}\right],\left[T_{0} U: T_{1} U: V\right]\right) \in \mathbb{F}_{1}$ to the point in $\mathbb{P}^{n}$ with coordinates $X_{M}=0, M \in C_{d}$, with $V_{l}=0, l=1, \ldots, n-\left(d^{2}+d+1\right)$, and with

$$
\begin{gathered}
Y_{0}=U T_{0}^{k}, \quad \ldots, \quad Y_{i}=U T_{0}^{k-i} T_{1}^{i}, \quad \ldots, \quad Y_{k}=U T_{1}^{k}, \\
Z_{0}=V T_{0}^{k-1}, \quad \ldots, \quad Z_{j}=V T_{0}^{k-1-j} T_{1}^{j}, \quad \ldots, \quad Z_{k-1}=V T_{1}^{k-1} .
\end{gathered}
$$

This is an embedding whose image $\Sigma=f\left(\mathbb{F}_{1}\right)$ is a rational normal scroll of degree $2 k-1$.

The pullback map $H^{0}\left(\left(\mathbb{P}^{2 k}, \mathcal{O}_{\mathbb{P}^{2 k}}(1)\right) \rightarrow H^{0}\left(\mathbb{F}_{1}, \mathcal{O}(1, k-1)\right)\right.$ is surjective by construction. The natural map

$$
\operatorname{Sym}^{d-1} H^{0}\left(\mathbb{F}_{1}, \mathcal{O}(1, k-1)\right) \longrightarrow H^{0}\left(\mathbb{F}_{1}, \mathcal{O}(d-1,(d-1)(k-1))\right)
$$

is surjective. Therefore the pullback map

$$
H^{0}\left(\mathbb{P}^{2 k}, \mathcal{O}_{\mathbb{P}^{2 k}}(d-1)\right) \longrightarrow H^{0}\left(\mathbb{F}_{1}, \mathcal{O}(d-1,(d-1)(k-1))\right)
$$

is surjective. For each monomial $M \in C_{d}$, choose a polynomial $G_{M}\left(Y_{0}, \ldots, Y_{2 k}\right)$ such that $f^{*} G_{M}=M$. 


\section{RATiONAL CURVES ON HYPERSURFACES, II}

Consider the hypersurface $X \subset \mathbb{P}^{n}$ with defining equation

$$
\begin{aligned}
& F=\quad \sum_{j=0}^{d-2} \quad\left(Y_{j} Z_{j+1}-Y_{j+1} Z_{j}\right) \quad Y_{0}^{d-2-j} Y_{k-3}^{i} \\
& +\sum_{j=2}^{d-2} \quad\left(Y_{d-1+j} Z_{d+j}-Y_{d+j} Z_{d-1+j}\right) \quad Y_{k}^{j-2} Y_{3}^{d-2-l} Z_{3} Z_{4} \\
& +\quad\left(Y_{d-1} Z_{d}-Y_{d} Z_{d-1}\right) \quad Y_{k-3}^{d-4} Y_{k-4}^{2} \\
& +\left(Y_{d} Z_{d+1}-Y_{d+1} Z_{d}\right) \quad Y_{1}^{d-4} Z_{7} Z_{8} \\
& +\quad\left(Y_{k-2} Z_{k-1}-Y_{k-1} Z_{k-2}\right) \quad Y_{k}^{d-4} Z_{k-1} Z_{5} \\
& +\left(Y_{k} Z_{0}-Z_{d} Y_{d}\right) \quad Z_{0}^{d-2} \\
& +\sum_{M \in C_{d}} G_{M}\left(Y_{0}, \ldots, Y_{2 k}\right) X_{M} \text {. }
\end{aligned}
$$

\begin{tabular}{|c|c|c|}
\hline Coordinate & $\operatorname{IN}\left(\partial_{X, \Sigma}(\right.$ coordinate $\left.)\right)$ & Lower-order terms in $\partial_{X, \Sigma}$ (coordinate) \\
\hline $\begin{array}{l}Z_{j+1} \\
j=0, \ldots, d-1\end{array}$ & $U^{d-1} T_{0}^{(k-1) d-(k-2) j} T_{1}^{(k-2) j}$ & $-U^{d-1} T_{0}^{(k-1) d-(k-2)(j+1)-1} T_{1}^{(k-2)(j+1)+1}$ \\
\hline$Z_{d}$ & $U^{d-1} T_{0}^{(k-1) d-(k-2)(d-1)} T_{1}^{(k-2)(d-1)}$ & $\begin{array}{l}-U^{d-3} V^{2} T_{0}^{(k-1)(d-2)+(d-3)} T_{1}^{k-1} \\
-U V^{d-2} T_{0}^{(k-1)(d-2)+d} T_{1}^{d}\end{array}$ \\
\hline $\begin{array}{l}Y_{j+1} \\
j=0, \ldots, d-1\end{array}$ & $-U^{d-2} V T_{0}^{(k-1) d-1-(k-2) j} T_{1}^{(k-2) j}$ & $+U^{d-2} V T_{0}^{(k-1) d-(k-2)(j+1)-2} T_{1}^{(k-2)(j+1)+1}$ \\
\hline$Y_{d}$ & $-U^{d-2} V T_{0}^{(k-1) d-1-(k-2)(d-1)} T_{1}^{(k-2)(d-1)}$ & $\begin{array}{l}+U^{d-4} V^{2} T_{0}^{(k-1)(d-2)+(d-4)} T_{1}^{k-1} \\
-V^{d-1} T_{0}^{(k-1)(d-1)-d_{1}} T_{1}^{d}\end{array}$ \\
\hline$Z_{d+j}$ & $U^{d-3} V^{2} \times$ & $-U^{d-2} V^{2} \times$ \\
\hline$j=1, \ldots, d-2$ & $T_{0}^{(k-1)(d-1)+(d-3)-(k-2) j} T_{1}^{(k-2) j}$ & $T_{0}^{(k-1)(d-1)+(d-4)+(k-2)(j+1)} T_{1}^{(k-2)(j+1)}$ \\
\hline$Z_{k-1}$ & $\begin{array}{l}U^{d-3} V^{2} \times \\
T_{0}^{(k-1)(d-1)+(d-3)-(k-2)(d-1)} T_{1}^{(k-2)(d-1)}\end{array}$ & \\
\hline$Y_{d+j}$ & $-U^{d-4} V^{3} \times$ & $U^{d-4} V^{3} \times$ \\
\hline$j=1, \ldots, d-2$ & $T_{0}^{(k-1)(d-1)+(d-4)-(k-2) j} T_{1}^{(k-2) j}$ & $T_{0}^{(k-1)(d-1)+(d-5)+(k-2)(j+1)} T_{1}^{(k-2)(j+1)}$ \\
\hline$Y_{k-1}$ & $\begin{array}{l}U^{d-4} V^{3} \times \\
T_{0}^{(k-1)(d-1)+(d-4)-(k-2)(d-1)} T_{1}^{(k-2)(d-1)}\end{array}$ & \\
\hline$Y_{k}$ & $V^{d-1} T_{0}^{(k-1)(d-1)}$ & \\
\hline $\begin{array}{l}X_{M} \\
M \in C_{d}\end{array}$ & $M$ & \\
\hline$Z_{0}$ & $-U^{d-1} T_{0}^{(k-1)(d-1)+(d-2)} T_{1}$ & $U V^{d-2} T_{0}^{(k-1)(d-2)} T_{1}^{k}$ \\
\hline$Y_{0}$ & $U^{d-2} V T_{0}^{(k-1)(d-1)+(d-3)} T_{1}$ & \\
\hline
\end{tabular}

Observe that $F$ is contained in the homogeneous ideal of $\Sigma$, i.e. $\Sigma \subset X$. The derivative map $\partial_{X, \Sigma}$ acts on the coordinates $Y_{j}, Z_{j}, X_{M}$ as in Table 1. Each of the monomials in $A_{d}$ occurs as the initial term of $\partial_{X, \Sigma}$ acting on one coordinate. For every coordinate except $Y_{0}$ and $Z_{0}$, the initial term of $\partial_{X, \Sigma}$ is one of the monomials in $A_{d}$. By Lemma 7.6, the image of $\partial_{X, \Sigma}$ is a $(k-3)$-generating linear system.

TABLE 1. The map $\partial_{X, \Sigma}$. 


\section{J. HARRIS AND J. STARR}

i) $d=3$ : Suppose that $d=3$ and $n \geqslant 3^{2}+3+1=13$. Denote by $B_{3}$ the set of $4 d-1=11$ monomials,

$$
\begin{aligned}
& B_{d}=\left\{\begin{array}{lllllll} 
& U^{2} & & T_{0}^{12-(k-2) j} & T_{1}^{(k-2) j} & \mid j=0,1,2
\end{array}\right\}
\end{aligned}
$$

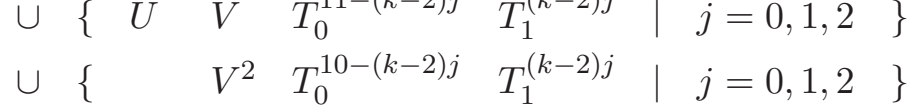

$$
\begin{aligned}
& \cup\left\{\begin{array}{llll}
U & V & T_{1}^{11}
\end{array}\right\} \\
& \cup\left\{V^{2} T_{1}^{10}\right\} .
\end{aligned}
$$

Denote by $C_{3}$ the singleton set consisting of the monomial $M=U^{2} T_{1}^{12}$. Denote by

$$
\left\{Y_{0}, \ldots, Y_{6}\right\} \cup\left\{Z_{0}, \ldots, Z_{5}\right\} \cup\left\{X_{M} \mid M \in C_{3}\right\} \cup\left\{V_{l} \mid l=1 \ldots, n-13\right\}
$$

a basis of $H^{0}\left(\mathbb{P}^{n}, \mathcal{O}_{\mathbb{P}^{n}}(1)\right)$, i.e. a basis of homogeneous coordinates on $\mathbb{P}^{n}$.

Denote by $f: \mathbb{F}_{1} \rightarrow \mathbb{P}^{12} \subset \mathbb{P}^{n}$ the morphism mapping $\left(\left[T_{0}: T_{1}\right],\left[T_{0} U: T_{1} U: V\right]\right) \in \mathbb{F}_{1}$ to the point in $\mathbb{P}^{n}$ with coordinates $X_{M}=0, M \in C_{d}$, with $V_{l}=0, l=1, \ldots, n-13$, and with

$$
\begin{array}{ccccc}
Y_{0}=U T_{0}^{6}, & \ldots, & Y_{i}=U T_{0}^{6-i} T_{1}^{i}, & \ldots, & Y_{k}=U T_{1}^{6}, \\
Z_{0}=V T_{0}^{5}, & \ldots, & Z_{j}=V T_{0}^{5-j} T_{1}^{j}, & \ldots, & Z_{5}=V T_{1}^{5} .
\end{array}
$$

This is an embedding whose image $\Sigma=f\left(\mathbb{F}_{1}\right)$ is a rational normal scroll of degree 11.

Consider the hypersurface $X \subset \mathbb{P}^{n}$ with defining equation

$$
\begin{aligned}
& F=\quad \sum_{j=0}^{1} \quad\left(Y_{j} Z_{j+1}-Y_{j+1} Z_{j}\right) \quad Y_{0}^{1-j} Y_{3}^{i} \\
& +\quad\left(Y_{2} Z_{3}-Y_{3} Z_{2}\right) \quad Y_{6} \\
& +\quad\left(Y_{5} Z_{5}-Y_{6} Z_{4}\right) \quad Z_{5} \\
& +\left(Y_{3} Z_{3}-Y_{6} Z_{0}\right) \quad Z_{4} \\
& +\quad\left(Y_{2} Z_{2}-Y_{4} Z_{0}\right) \quad Z_{0} \\
& +\quad Y_{6}^{2} X_{M} \text {. }
\end{aligned}
$$

Observe that $F$ is contained in the homogeneous ideal of $\Sigma$, i.e. $\Sigma \subset X$. It is straightforward to compute the action of $\partial_{X, \Sigma}$ on the coordinates $Y_{j}, Z_{j}, X_{M}$. Every monomial in $A_{3}$ occurs as the initial term of $\partial_{X, \Sigma}$ acting on one coordinate. For every coordinate except $Y_{0}$ and $Z_{0}$, the initial term of $\partial_{X, \Sigma}$ is one of the monomials in $A_{3}$. By Lemma 7.6, the image of $\partial_{X, \Sigma}$ is a $(k-3)$-generating linear system.

ii) $d=2$ : Suppose that $d=2$ and $n \geqslant 2^{2}+2+1=7$. Let $k=3$. Denote by $B_{2}$ the set of six monomials,

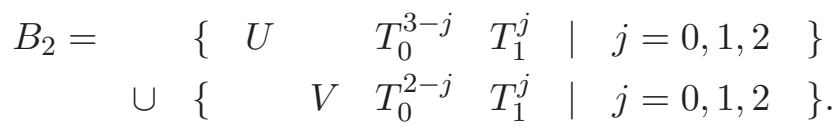

Denote by $C_{2}$ the singleton set consisting of the monomial $M=U T_{1}^{3}$. Denote by

$$
\left\{Y_{0}, Y_{1}, Y_{2}, Y_{3}, Z_{0}, Z_{1}, Z_{2}, X_{M}\right\} \cup\left\{V_{l} \mid l=1, \ldots, n-7\right\}
$$

a basis of $H^{0}\left(\mathbb{P}^{n}, \mathcal{O}_{\mathbb{P}^{n}}(1)\right)$, i.e. a basis of homogeneous coordinates on $\mathbb{P}^{n}$.

Denote by $f: \mathbb{F}_{1} \rightarrow \mathbb{P}^{6} \subset \mathbb{P}^{n}$ the morphism mapping $\left(\left[T_{0}: T_{1}\right],\left[T_{0} U: T_{1} U: V\right]\right) \in \mathbb{F}_{1}$ to the point in $\mathbb{P}^{n}$ with coordinates $X_{M}=0, M \in C_{d}$, with $V_{l}=0, l=1, \ldots, n-7$, and with

$$
\begin{gathered}
Y_{0}=U T_{0}^{3}, \quad Y_{1}=U T_{0}^{2} T_{1}, \quad Y_{2}=U T_{0} T_{1}^{2}, \quad Y_{3}=U T_{1}^{3}, \\
Z_{0}=V T_{0}^{2}, \quad Z_{1}=V T_{0} T_{1}, \quad Z_{2}=V T_{1}^{2} .
\end{gathered}
$$

This is an embedding whose image $\Sigma=f\left(\mathbb{F}_{1}\right)$ is a rational normal scroll of degree 6 . 


\section{RATIONAL CURVES ON HYPERSURFACES, II}

Consider the hypersurface $X \subset \mathbb{P}^{n}$ with defining equation

$$
F=\left(Y_{3} Z_{1}-Y_{2} Z_{2}\right)+\left(Y_{0} Z_{1}-Y_{1} Z_{0}\right)+Y_{3} X_{M}
$$

Observe that $F$ is contained in the homogeneous ideal of $\Sigma$, i.e. $\Sigma \subset X$. It is straightforward to compute the action of $\partial_{X, \Sigma}$ on the coordinates $Y_{j}, Z_{j}, X_{M}$. Every monomial in $A_{2}$ occurs as the initial term of $\partial_{X, \Sigma}$ acting on one coordinate. For every coordinate except $Y_{0}$, the initial term of $\partial_{X, \Sigma}$ is one of the monomials in $A_{2}$. By Lemma 7.6, the image of $\partial_{X, \Sigma}$ is a $(k-3)$-generating linear system.

ii) $d=1$ : Denote by

$$
\left\{Y_{0}, Y_{1}, Y_{2}, Y_{3}, Z_{0}, Z_{1}, Z_{2}, X_{M}\right\} \cup\left\{V_{l} \mid l=1, \ldots, n-7\right\}
$$

a basis of $H^{0}\left(\mathbb{P}^{n}, \mathcal{O}_{\mathbb{P}^{n}}(1)\right)$, i.e. a basis of homogeneous coordinates on $\mathbb{P}^{n}$.

Denote by $f: \mathbb{F}_{1} \rightarrow \mathbb{P}^{6} \subset \mathbb{P}^{n}$ the morphism mapping $\left(\left[T_{0}: T_{1}\right],\left[T_{0} U: T_{1} U: V\right]\right) \in \mathbb{F}_{1}$ to the point in $\mathbb{P}^{n}$ with coordinates $X_{M}=0, M \in C_{d}$, with $V_{l}=0, l=1, \ldots, n-7$, and with

$$
\begin{gathered}
Y_{0}=U T_{0}^{3}, \quad Y_{1}=U T_{0}^{2} T_{1}, \quad Y_{2}=U T_{0} T_{1}^{2}, \quad Y_{3}=U T_{1}^{3}, \\
Z_{0}=V T_{0}^{2}, \quad Z_{1}=V T_{0} T_{1}, \quad Z_{2}=V T_{1}^{2} .
\end{gathered}
$$

This is an embedding whose image $\Sigma=f\left(\mathbb{F}_{1}\right)$ is a rational normal scroll of degree 6 .

Consider the hypersurface $X \subset \mathbb{P}^{n}$ with defining equation $F=X_{M}$. Observe that $F$ is contained in the homogeneous ideal of $\Sigma$, i.e. $\Sigma \subset X$. The image of $\partial_{X, \Sigma}\left(X_{M}\right)$ is a generator for $S_{0,0}$; i.e. the image of $\partial_{X, \Sigma}$ is a $(k-3)$-generating linear system.

Together with Remark 7.1, Lemma 7.11 and Proposition 7.12 imply the following corollary.

Corollary 7.13. If $X \subset \mathbb{P}^{n}$ is a general hypersurface of degree $d$ and if $n \geqslant d^{2}+d+1$, then there exists a 1-morphism $\zeta: C_{0} \rightarrow \overline{\mathcal{M}}_{0,1}(X, 1)$ that is both very twisting and very positive.

\section{Proof of the main theorem}

As explained at the end of $\S 1$, if $d<(n+1) / 2$, then for a general hypersurface $X \subset \mathbb{P}^{n}$ of degree $d$, Hypotheses 1.5, 1.6 and 1.7 are satisfied. By Corollary 6.6, if $d \geqslant 2$ and $n+1 \geqslant d^{2}$, or if $d=1$ and $n \geqslant 3$, then for a general hypersurface $X \subset \mathbb{P}^{n}$ of degree $d$, Hypothesis 4.10 is satisfied. Finally, if $n \geqslant d^{2}+d+1$ then by Corollary 7.13 there exists a very twisting, very positive family $\zeta: C_{0} \rightarrow \overline{\mathcal{M}}_{0,1}(X, 1)$. Therefore $(\zeta, \zeta)$ is an inducting pair.

By Theorem 5.13, for every $e \geqslant 1$ there exists an inducting pair $\left(\zeta_{1}, \bar{\zeta}_{e}\right)$. In particular, there exists a very positive 1-morphism $\bar{\zeta}_{e}: C \rightarrow \overline{\mathcal{M}}_{0,1}(X, e)$. As shown in the proof of Theorem 5.13 , it may be assumed that $C$ is smooth and that the image of $C$ is contained in the smooth locus of the fine moduli locus. By Lemma 4.14, part $\mathrm{i}, \bar{\zeta}_{e}$ is a very free morphism. By [HRS04, Proposition 7.4], $\overline{\mathcal{M}}_{0,0}(X, e)$ is an irreducible variety. Therefore, by $\left[\operatorname{Kol} 96\right.$, Theorem IV.3.7], $\overline{\mathcal{M}}_{0,0}(X, e)$ is rationally connected.

\section{ACKNowledgements}

We are very grateful to A. J. de Jong and Steven Kleiman for many useful conversations. We are also grateful to the referees for their many useful comments. 


\section{RATIONAL CURVES ON HYPERSURFACES, II}

\section{REFERENCES}

Ale96 V. Alexeev, Moduli spaces $M_{g, n}(w)$ for surfaces, in Higher dimensional complex varieties (Trento, 1994) (de Gruyter, Berlin, 1996).

AV02 D. Abramovich and A. Vistoli, Compactifying the space of stable maps, J. Amer. Math. Soc. 15 (2002), 27-75.

Beh97 K. Behrend, Gromov-Witten invariants in algebraic geometry, Invent. Math. 127 (1997), 601-617.

BF98 K. Behrend and B. Fantechi, The intrinsic normal cone, Invent. Math. 128 (1998), 45-88.

BM96 K. Behrend and Y. Manin, Stacks of stable maps and Gromov-Witten invariants, Duke Math. J. 85 (1996), 1-60.

BLR90 S. Bosch, W. Lütkebohmert and M. Raynaud, Néron models, Ergebnisse der Mathematik und ihrer Grenzgebiete, vol. 2 (Springer, Berlin, 1990).

Deb01 O. Debarre, Higher-dimensional algebraic geometry, Universitext (Springer, Berlin, 2001).

FP95 W. Fulton and R. Pandaharipande, Notes on stable maps and quantum cohomology, in Algebraic geometry - Santa Cruz 1995 (American Mathematical Society, Providence, RI, 1995), 45-96.

Ful98 W. Fulton, Intersection theory, Ergebnisse der Mathematik und ihrer Grenzgebiete (3), vol. 2 (Springer, Berlin, 1998), second edition.

GD64 A. Grothendieck and J. Dieudonné, Éléments de géométrie algébrique, IV, Publ. Math. Inst. Hautes Études Sci. 20, 24, 28, 32 (1964-1967).

Har66 R. Hartshorne, Residues and duality, Lecture notes of a seminar on the work of A. Grothendieck, Lecture Notes in Mathematics, vol. 20 (Springer, Berlin, 1966).

Har77 R. Hartshorne, Algebraic geometry, Graduate Texts in Mathematics, vol. 52 (Springer, Berlin, 1977).

HRS04 J. Harris, M. Roth and J. Starr, Rational curves on hypersurfaces of low degree, I, J. reine angew. Math. 571 (2004), 73-106.

Jou83 J.-P. Jouanolou, Théorèmes de Bertini et applications, Progress in Mathematics, vol. 42 (Birkhäuser, Basel, 1983).

KM98 J. Kollár and S. Mori, Birational geometry of algebraic varieties, Cambridge Tracts in Mathematics, vol. 134 (Cambridge University Press, Cambridge, 1998).

Kol96 J. Kollár, Rational curves on algebraic varieties, Ergebnisse der Mathematik und ihrer Grenzgebiete (3), vol. 32 (Springer, Berlin, 1996).

KP01 B. Kim and R. Pandharipande, The connectedness of the moduli space of maps to homogeneous spaces, in Symplectic geometry and mirror symmetry (World Scientific, Singapore, 2001), ch. 5.

Lan52 S. Lang, On quasi-algebraic closure, Ann. of Math. (2) 55 (1952), 373-390.

Pan99 R. Pandharipande, Intersections of Q-divisors on Kontsevich's moduli space, Trans. Amer. Math. Soc. 351 (1999), 1481-1505.

Joe Harris harris@math.harvard.edu

Department of Mathematics, Harvard University, Cambridge, MA 02138, USA

Jason Starr jstarr@math.mit.edu

Department of Mathematics, Massachusetts Institute of Technology, Cambridge, MA 02139, USA 